\title{
Optimal dynamic matching
}

\author{
MARIAGIOVANNA BACCARA \\ Olin School of Business, Washington University in St. Louis \\ SANGMoK LeE \\ Department of Economics, Washington University in St. Louis \\ LEEAT YARIV \\ Department of Economics, Princeton University
}

\begin{abstract}
We study a dynamic matching environment where individuals arrive sequentially. There is a trade-off between waiting for a thicker market, allowing for higherquality matches, and minimizing agents' waiting costs. The optimal mechanism cumulates a stock of incongruent pairs up to a threshold and matches all others in an assortative fashion instantaneously. In discretionary settings, a similar protocol ensues in equilibrium, but expected queues are inefficiently long. We quantify the welfare gain from centralization, which can be substantial, even for low waiting costs. We also evaluate welfare improvements generated by alternative priority protocols.
\end{abstract}

KeYwords. Dynamic matching, mechanism design, market design, child adoption, organ donation.

JEL CLASSification. C61, C78, D47.

\section{INTRODUCTION}

\subsection{Overview}

Many matching processes are inherently dynamic, with participants arriving and matches being created over time. For instance, in the child-adoption process, parents and children arrive steadily-data from one U.S. adoption facilitator who links adoptive parents and birth mothers willing to relinquish children for adoption indicates a rate of about 11 new potential adoptive parents and 13 new birth mothers entering the facilitator's operation each month. ${ }^{1}$ While the overall statistics on the entry of parents and children into the U.S. adoption process are not well documented, adoption touches

Mariagiovanna Baccara: mbaccara@wustl . edu

SangMok Lee: sangmoklee@wustl . edu

Leeat Yariv: lyariv@princeton. edu

We thank Johannes Horner, George Mailath, Andy Postlewaite, and Larry Samuelson for very helpful comments. We gratefully acknowledge financial support from the National Science Foundation through grants SES 0963583 and SES 1629613.

${ }^{1}$ This adoption facilitator is one of 25 registered in its state of operation. See Baccara et al. (2014) for details.

(C) 2020 The Authors. Licensed under the Creative Commons Attribution-NonCommercial License 4.0. Available at https://econtheory.org. https://doi.org/10.3982/TE3740 
upon many lives: The Census 2010 indicates that about 1.5 million or $2.4 \%$ of all children have been adopted. Likewise, many labor markets entail unemployed workers and job openings that become available at different periods. A similar picture emerges when considering organ donation. According to the Organ Donation and Transplantation Statistics, a new patient is added to the kidney transplant list every 14 minutes and about 3000 patients are added to the kidney transplant list each month. A significant fraction of transplants are carried out using live donors-in 2014, about a third of approximately 17,000 kidney transplants that took place in the U.S. involved such donors.

Nonetheless, by and large, the extant matching literature has taken a static approach to market design - participants all enter at the same time and the market's operations are restricted in their horizon (see the literature review below for several important exceptions). In the current paper, we offer techniques for extending that approach to dynamic settings.

All of the examples mentioned above share two important features. First, match quality varies and agents care about whom they match with. Second, waiting for a match is costly, be it for financial costs of keeping lawyers on retainer for potential adoptive parents, children's hardship from growing older in the care of social services, the lack of wages and needed employees in labor markets, or medical risks for organ patients and psychological waiting costs for donors. These two features introduce a crucial trade-off. On the one hand, a thick market can help generate a greater match surplus; on the other hand, a thin market allows for quicker matching and cuts down on waiting costs. The goal of this paper is to characterize the resolution of this trade-off in both centralized and relatively more decentralized environments. Namely, we identify the optimal protocol by which a social planner would match agents over time. We also identify conditions under which discretionary matching processes would especially benefit from centralized intervention using the optimal protocol.

Specifically, we consider a market that evolves dynamically. There are two classes of agents, which we refer to as "squares" and "rounds." At each period, a pair consisting of a square and a round enters the market. Squares and rounds each have two types, one type more desirable than the other. For instance, if we think of squares and rounds as children relinquished for adoption and potential adoptive parents, types can stand for gender of children and wealth levels of potential adoptive parents, respectively (see Baccara et al. 2014). Alternatively, if we think of the two classes of agents as workers and firms, worker types can correspond to skills and firm types can correspond to various benefit packages offered. We assume that preferences are supermodular so that the (market-wide) assortative matching maximizes joint welfare. We also assume that, once agents arrive at the market, waiting before being matched comes at a per-period cost.

We start by analyzing the optimal matching mechanism in such settings, the mechanism that maximizes the expected per-period payoffs for market participants. We show that the optimal mechanism takes a simple form. Whenever congruent pairs of agents - a square and round that are of the same type-are present in the market, they are matched instantaneously. When only incongruent agents are present in the market, they are held in a queue. When the stock of incongruent pairs in the queue exceeds a certain threshold, they are matched in sequence, until the queue length falls back within 
the threshold. Such thresholds induce a Markov process, where states correspond to the length of queues of incongruent agent pairs. Any threshold yields a different steadystate distribution over possible queue lengths. We evaluate the expected welfare of such threshold mechanisms in the steady state. The optimal mechanism utilizes the threshold that maximizes welfare. When waiting costs are vanishingly small, the welfare under the optimal mechanism approaches the maximum feasible, that generated by no matches of incongruent pairs. As waiting costs increase, the welfare generated by the optimal mechanism decreases.

This welfare decrease raises the question of the value of dynamic clearinghouses for non-trivial waiting costs in different environments, identified by type distributions and preferences. We therefore study the performance of a simple discretionary matching process in our setting. As before, we consider agents arriving at the market in sequence. At each period, agents in the market declare their willingness to match with partners of either type. After these demands have been made, the maximal number of pairs of willing agents are matched in order of arrival (first-in-first-out, or FIFO protocol). ${ }^{2}$ Those who prefer to stay in the market, or have to stay for lack of willing partners, form a queue. ${ }^{3}$ In our environment, desirable individuals waiting in the market impose three types of externalities. First, they impose a longer wait and potentially missed desirable matches on those that follow them in the queue. Second, they prevent undesirable agents present in the market from matching immediately. Last, they impose a positive externality on desirable agents on the other side of the market, who are more likely to get a quicker match with a preferable partner. As it turns out, the negative externalities of waiting dominate and lead to excessive waiting in the discretionary setting. In fact, the matching protocol induced by equilibrium in the discretionary matching process ends up resembling the protocol corresponding to the optimal mechanism, but with higher thresholds for the queues' lengths.

We evaluate the difference in welfare generated by a centralized and a discretionary process as a function of the environment's primitives-agents' type distribution and waiting costs. Regarding the type distribution, as the frequency of desirable types increases, the option value of waiting becomes higher and the wedge between the performance of the centralized and discretionary processes grows. The comparative statics with respect to waiting costs are more subtle. An increase in the cost of waiting has a direct and indirect effect. The direct effect is due to the longer expected queues in the discretionary setting. Fixing the expected queue lengths corresponding to both processes, an increase in per-period waiting costs has a multiplier effect-the generated welfare differential is the difference between the expected time agents wait in queue under the two processes, multiplied by the change in costs. The indirect effect is that both the optimal threshold as well as the equilibrium threshold in the discretionary process decrease with waiting costs. The difference between these two thresholds therefore narrows as costs increase, which mutes the welfare gap between the two processes. We show that the combination of these effects leads to a welfare wedge that is locally increasing in

\footnotetext{
${ }^{2}$ This process is reminiscent of a double auction, as each agent submits a "demand function" specifying that types of agents she would be interested in matching with immediately.

${ }^{3}$ The process is individually rational for all under preference restrictions we provide.
} 
costs, but exhibits a general decreasing trend. Ultimately, when costs are prohibitively high, both processes lead to instantaneous matches and identical welfare levels.

The discretionary process we focus on relies on the FIFO protocol. While this seems to approximate many real-life decentralized processes, we also analyze a discretionary setting governed by a last-in-first-out (LIFO) priority protocol. We show that this alternative protocol also generates excessive waiting, but less so than the FIFO protocol. Finally, we consider an extension of our model to general asymmetric environments.

\subsection{Related literature}

The interest surrounding dynamic matching is recent and the literature on this topic is still relatively limited. Much of this literature stemmed from the organ-donation application, and has therefore focused on compatibility-based preferences. In contrast, we aim to speak to applications-such as child adoption and labor markets-in which agents on both sides of the market have preferences over potential matches.

Zenios (1999) develops a queueing model to explain the differences between waiting times of different categories of patients anticipating a kidney transplant. In the context of kidney exchange, Ünver (2010) focuses on a market in which donors and recipients arrive stochastically, and the central planner's objective is to minimize total discounted waiting costs. He shows that when multiway matches are possible, some twoway matches could be optimally withheld in order to allow future multiway matches. ${ }^{4}$

Akbarpour et al. (2020), like us, inspect the benefits of different mechanisms in a dynamic matching environment. In their setting, however, preferences are based on compatibility. Agents in the system become "critical" at random dates, and perish immediately if they are unmatched. Therefore, when waiting costs are negligible, the goal of the planner is to minimize the number of perished agents. Market thickness is beneficial in that it guarantees the availability of immediate matches for agents who become critical. In contrast with our setting, left to their own devices, agents would match quickly and useful mechanisms induce agents to wait. While the welfare benchmark in Akbarpour et al. (2020) is that of an omniscient planner, our different focus allows us to fully characterize the optimal mechanism, which serves as our benchmark for welfare comparisons.

Loertscher et al. (2018) follow up on our paper and focus on the optimal mechanism when the planner and participants discount future utilities. The interpretation of the objective function in their setting is subtle. When two agents of identical types who arrived at different times are matched at date $t$, the agents experience different discounted utilities, but the planner's utility from the two matches is identical. Similarly, when assessing future matches, the planner cares only about when they are formed. That is, individual costs of delay do not enter the planner's objective function. Doval and Szentes (2019) also follow up on this paper and consider a planner who benefits from the discounted utility agents receive in steady state. In their setting, once agents have been in the market for a while, their impact on welfare becomes vanishingly small. The planner is then

\footnotetext{
${ }^{4}$ Some recent models in inventory control have a similar flavor to the compatibility-based matching process considered by Ünver (2010); see, e.g., Gurvich and Ward (2014) and Hu and Zhou (2018).
} 
willing to "store" agents for a long while in hopes of them allowing new arrivals to match quickly. While the fully-optimal mechanism is not analyzed, results suggest that, naturally, the planner may impose more waiting on individuals than they themselves would in a discretionary FIFO protocol.

Dynamic assignments, where only one side of the market has agency, have received attention in the queueing literature. For instance, Naor (1969) illustrates that individuals who decide whether to join a FIFO queue for some service may wait excessively. Waiting agents impose a purely negative externality by increasing expected wait time of others joining the queue. Hassin (1985) shows that a LIFO queue yields equilibrium behavior that emulates the socially optimal. The negative externality in these papers is also present in ours. However, the two-sided nature of our setting introduces additional positive externalities. Consequently, the analysis of our decentralized process is quite different. In particular, LIFO protocols do not generally yield socially-optimal outcomes.

Related, Leshno (2019) studies a one-sided market in which potentiallyheterogeneous objects are allocated to agents who wait in a queue. With incomplete information on agents' preferences, a policy that introduces delays can be beneficial. ${ }^{5}$ Anderson et al. (2017) study an environment in which each agent is endowed with an item that can be exchanged with an item owned by someone else and compatibility is stochastic. They find that a policy that maximizes immediate exchanges performs nearly optimally. ${ }^{6,7}$

There is recent work that studies discretionary matching processes that are dynamic, considering both informational and time frictions (e.g., Ferdowsian et al. 2020, Haeringer and Wooders 2011, and Pais 2008). In that literature, the number of agents on each side of the market is fixed at the outset, but interactions occur over time. Time and information frictions constitute obstacles to stability.

The search and matching literature is also related (e.g., Burdett and Coles 1997, Eeckhout 1999, and the survey by Rogerson et al. 2005). There, each period, workers and firms randomly encounter each other and decide jointly whether to pursue a match and leave the market or separate and wait further. With assortative preferences, as time frictions vanish, generated outcomes approximate a stable matching. A crucial difference with our setting is the market's stationarity-the perceived distribution of potential partners does not change with time, and each side of the market solves an option value problem.

Last, a large literature considers dynamic matching of buyers and sellers and inspects protocols that increase efficiency or allow for Walrasian equilibrium outcomes to emerge with increasingly patient agents (see, e.g., Satterthwaite and Shneyerov 2007 and Taylor 1995).

\footnotetext{
${ }^{5}$ See also Bloch and Cantala (2017), Ortoleva et al. (2020), and Schummer (2019).

${ }^{6}$ On the benefits of creating thicker pools in sparse dynamic allocation settings, see also Ashlagi et al. (2014), Ashlagi et al. (2019), and references there.

${ }^{7}$ While most of this literature has focused on market design, Doval (2020) introduces a notion of stability in dynamic environments and provides conditions under which dynamically stable allocations exist.
} 


\section{SETUP}

We study an infinite-horizon dynamic matching market. There are two kinds of agents: squares and rounds. Squares and rounds can stand for potential adoptive parents and children relinquished for adoption, workers and employers, patients and (good samaritan) donors, etc. At each time $t \in\{1,2, \ldots\}$, one square and one round arrive at the market. Each square can be of either type "high" $(H)$ or "low" $(L)$ with probability $p$ or $1-p$, respectively, and each round can be of type "high" $(h)$ or "low" $(l)$ with probability $p$ or $1-p$, respectively. These types correspond to the attributes of participants-they can stand for the wealth of parents and race of children in the adoption application, level of education of employees, and social benefits or promotion likelihoods for employers in labor markets, age or tissue types in the organ donation context, etc. ${ }^{8}$

In our model, squares seek to match with rounds and vice versa. We denote by $U_{x}(y)$ the surplus for a type- $x$ participant from matching with a type- $y$ participant. We assume that preferences are assortative: $H$-squares are more desirable for all rounds and $h$-rounds are more desirable for all squares. That is,

$$
\begin{array}{ll}
U_{H}(h)>U_{H}(l), & U_{L}(h)>U_{L}(l), \\
U_{h}(H)>U_{h}(L), & U_{l}(H)>U_{l}(L) .
\end{array}
$$

It will be convenient to denote:

$$
\begin{aligned}
U_{H h} & \equiv U_{H}(h)+U_{h}(H), \quad U_{H l} \equiv U_{H}(l)+U_{l}(H), \\
U_{L h} & \equiv U_{L}(h)+U_{h}(L), \quad U_{L l} \equiv U_{L}(l)+U_{l}(L),
\end{aligned}
$$

as well as

$$
U \equiv U_{H h}+U_{L l}-U_{H l}-U_{L h}
$$

We will further assume that $U>0$ so that the utilitarian-efficient matching in a static market creates the maximal number of $(H, h)$ and $(L, l)$ pairs. The value of $U$ captures the efficiency gain from the assortative matching relative to the antiassortative matching. Notice that $U>0$ is tantamount to assuming supermodular assortative preferences (à la Becker 1974) and $U$ can be thought of as the degree of supermodularity preferences exhibit. We assume that each square and round suffer a cost $c>0$ for each period they spend on the market waiting to be matched, and that agents leave the market only by matching. ${ }^{9}$

\footnotetext{
${ }^{8}$ In some markets, wages differ across individual employees and can be thought of as transfers, which this paper does not handle. However, Hall and Krueger (2012) suggest that a large fraction of jobs have posted wages that do not appear to reflect general equilibrium wages tailored to the precise composition of the market. Our model speaks to this segment of the market.

${ }^{9}$ In the working-paper version, Baccara et al. (2018), we provide bounds on agents' utility from remaining unmatched that assure this assumption is consistent with individual rationality. Specifically, we show that individual rationality holds when all agents are acceptable and when any $l$-round receives a utility lower than $U_{l}(L)-\frac{p}{1-p}\left[U_{h}(H)-U_{h}(L)\right]$ when leaving the market unmatched (analogously for $L$-squares).
} 
Several assumptions merit discussion. We assume that preferences are supermodular and that waiting costs are identical for squares and rounds for presentation simplicity. These assumptions are common in the literature, and as we describe in Section 5, lead to a conservative comparison of the optimal and discretionary matching protocols. $^{10}$

The assumption that the distribution of round types mirrors that of squares also simplifies our analysis. It implies that if we drew a large population of rounds and squares, the realized distributions of types would be approximately balanced with high probability. This may be a fairly reasonable assumption for certain applications, such as organ donation. Indeed, the literature does not report differences in tissue-type distributions of donors and patients. ${ }^{11}$ Our assumption also approximately holds for certain attributes in the online-dating world (see Hitch et al. 2010). The techniques we introduce can, however, be used even without this symmetry assumption. In Section 7, we discuss how to replicate some of our analysis for general asymmetric environments.

In our setting, a pair of agents arrives at the market in each period. The analysis would remain virtually identical were we to assume that pairs arrive at random times following, say, a Poisson distribution. Moreover, our results extend directly to markets with multiple pairs arriving each period. The assumption that participants arrive in pairs is, however, important for our techniques. It ensures that the market is balanced throughout the matching process. It is a reasonable assumption for some applications. For example, in the adoption process, Baccara et al. (2014) follow one adoption facilitator over several years. They document a similar volume of potential adoptive parents and children available for adoption appearing each month. ${ }^{12}$

In our model, agents incur a fixed cost $c$ for every period they spend unmatched. Additive waiting costs are a natural way to capture flow costs that agents incur while unmatched in the system. For example, in the child-adoption setting, families seeking to adopt a child retain attorneys until a match is found. Similarly, pregnant women planning to relinquish their newborns for adoption are responsible for their own living and

\footnotetext{
${ }^{10}$ Our analysis carries through fully if participants are horizontally differentiated. In particular, if $H$ squares and $h$-rounds prefer one another and $L$-squares and $l$-rounds prefer one another, the utilitarianefficient static matching is assortative without further assumptions. This may be relevant in some childadoption contexts if both adoptive parents and birth mothers display what is often termed "homophilic preferences," preferring to match with individuals of their own race. We describe our results assuming assortative preferences since they are a leading example in the extant literature and potentially tie to more applications.

${ }^{11}$ Furthermore, the age of a donor is known to have a strong impact on the expected survival of a graft (see, e.g., Gjertson 2004 and Øien et al. 2007) and younger recipients have been suggested as the natural recipients of higher-quality organs (see Stein 2011). Our assumptions then fit a world in which both patients and donors are classified as "young" or "mature" and patients' and donors' age distributions are similar.

${ }^{12}$ Allowing for independent arrivals of squares and rounds introduces new challenges. Indeed, consider a symmetric environment in which a square arrives with probability $q$ each period and, similarly, a round arrives with probability $q$ each period. In this setting, there is a probability that a long queue of squares (or rounds) would form with no round (or square) available, of whichever type. In contrast with the setting that we study in the paper, for any fixed value of the outside option, the optimal mechanism involves retiring agents from the market without matches when a sufficiently long queue of individuals of their type is formed.
} 
medical costs during the pregnancy until matched, when the agreed-upon adoptive parents often cover these expenses. An alternative way to model waiting costs would be to consider agents' payoffs as discounted match utilities. As a first step, our criterion allows us for greater tractability. To see why, notice that, in the presence of discounting, the benefits of matching an agent would depend on the number of periods that agent already spent on the market. The relevant state space for the designer would then be vast. Also, the randomness inherent in the environment suggests that the timing of matches is potentially a random variable. Keeping track of expected exponentially discounted values then introduces nontrivial complications. ${ }^{13}$

Last, we assume there are only two types of squares and rounds for tractability. While this corresponds to a coarse description of many applications, the insights delivered by our binary-type analysis are useful for tackling environments with richer type sets (for more details, see Baccara et al. 2018).

\section{Optimal DYNAMic MATCHING}

\subsection{The matching process}

At any time $t \in\{1,2, \ldots\}$, after a new square-round pair enters the market, a queue corresponds to a vector $\mathbf{s}^{t}=\left(s_{H}, s_{L}, s_{h}, s_{l}\right)$, where each entry is the stock of squares or rounds of a particular type waiting in line. We represent the profile of matches created at time $t$ by the vector $\mathbf{m}^{t}=\left(m_{H h}, m_{H l}, m_{L h}, m_{L l}\right)$. For every $\mathbf{s}^{t} \in \mathbb{Z}_{+}^{4}$, a match profile $\mathbf{m}^{t} \in \mathbb{Z}_{+}^{4}$ has to satisfy a feasibility condition

$$
\begin{array}{cl}
m_{x h}+m_{x l} \leq s_{x} & \text { for } x \in\{H, L\}, \\
m_{H y}+m_{L y} \leq s_{y} & \text { for } y \in\{h, l\} .
\end{array}
$$

The surplus generated by the matches is

$$
S(\mathbf{m}) \equiv \sum_{(x, y) \in\{H, L\} \times\{h, l\}} m_{x y} U_{x y} .
$$

We denote the volume of remaining agents by $\mathbf{k}^{t}=\left(k_{H}, k_{L}, k_{h}, k_{l}\right)$, where

$$
\begin{array}{ll}
k_{x}=s_{x}-\left(m_{x h}+m_{x l}\right) & \text { for } x \in\{H, L\}, \\
k_{y}=s_{y}-\left(m_{H y}+m_{L y}\right) & \text { for } y \in\{h, l\} .
\end{array}
$$

The total waiting costs incurred by the remaining agents in period $t$ are then

$$
C(\mathbf{s}, \mathbf{m}) \equiv c\left(\sum_{x \in\{H, L, h, l\}} k_{x}\right) .
$$

Finally, the welfare generated at time $t$ is

$$
w(\mathbf{s}, \mathbf{m}) \equiv S(\mathbf{m})-C(\mathbf{s}, \mathbf{m}),
$$

\footnotetext{
${ }^{13}$ See Section 1.2 for a discussion of several follow-up papers to ours that introduce discounting without fully characterizing the optimal mechanism.
} 
if the profile of matches $\mathbf{m}$ is feasible given the stock $\mathbf{s}$, and $w(\mathbf{s}, \mathbf{m})=-\infty$ otherwise. At time $t+1$, the queue $\mathbf{s}^{t+1}$ is determined by the number of remaining agents $\mathbf{k}^{t}$ and the types of agents arriving at $t+1$. As an initial condition, we have $\mathbf{k}^{0}=(0,0,0,0)$. A mechanism $\mu$ is any rule governing matching profiles. We evaluate a mechanism by considering the average welfare it generates:

$$
v(\mu) \equiv \liminf _{T \rightarrow \infty} \frac{1}{T} E_{\mu}\left[\sum_{t=1}^{T} w\left(\mathbf{s}^{t}, \mathbf{m}^{t}\right)\right] .
$$

For any mechanism $\mu, v(\mu) \in \mathbb{R} \cup\{-\infty\}$, and the average welfare is bounded above by $U_{H h}$. This criterion allows us to focus on the long-run performance of mechanisms. We say that $\mu^{*}$ is optimal if $v\left(\mu^{*}\right)=\sup _{\mu} v(\mu) .^{14}$

We will consider the class of mechanisms that satisfy the following two assumptions. Restricting attention to mechanisms that satisfy Assumptions 1 and 2 implies no loss of generality, but simplifies the presentation (see Lemma A1 in the Appendix for details).

Assumption 1. $(H, h)$ and $(L, l)$ pairs are matched as soon as they become available.

Assumption 2. No more than $\frac{U}{2 c}$ squares (and rounds) are ever held in the market.

Assumption 1 requires an immediate match of congruent pairs. Intuitively, the only reason to hold on to, say, an $(H, h)$ pair is to create future $(H, l)$ or $(L, h)$ pairs. However, super-modularity implies that this is inferior to matching immediately the $(H, h)$ pair and then matching the future $(L, l)$ pair. To understand Assumption 2, recall that $U$ captures the extent of supermodularity of preferences-the utilitarian benefit of matching congruent over incongruent pairs. Suppose more than $U / 2 c$ squares (equivalently, rounds) are held in the market. This implies that at least one pair has been waiting for more than $U / 2 c$ periods. The utility benefit for that pair is at most $U$, while the perperiod cost is $2 c$. It would have been more efficient to match that pair immediately. Assumptions 1 and 2 guarantee that the relevant state space, corresponding to possible queue realizations, is finite. Standard techniques (see Ross 2014, and details in the Appendix) allow us to focus on the set of stationary and deterministic mechanisms (SDmechanisms). The matches created by a SD-mechanism $\mu^{\mathrm{SD}}: \mathbb{Z}_{+}^{4} \rightarrow \mathbb{Z}_{+}^{4}$ at every period depend only on the queue in place at that period.

\subsection{Structure of optimal dynamic mechanisms}

Assumptions 1 and 2 imply that, at any point in time, an optimal dynamic mechanism entails queues of only $H$-squares and $l$-rounds, or only $L$-squares and $h$-rounds. That

\footnotetext{
${ }^{14}$ This is a fairly standard approach; see, e.g., Ross (2014). We could have, in fact, applied an even stronger notion of optimality following Puterman (2005), where a mechanism $\mu$ would be called "optimal" if

$$
\liminf _{T \rightarrow \infty} \frac{1}{T} E_{\mu}\left[\sum_{t=1}^{T} w\left(\mathbf{s}^{t}, \mathbf{m}^{t}\right)\right] \geq \limsup _{T \rightarrow \infty} \frac{1}{T} E_{\mu^{\prime}}\left[\sum_{t=1}^{T} w\left(\mathbf{s}^{t}, \mathbf{m}^{t}\right)\right]
$$

for any other mechanism $\mu^{\prime}$.
} 


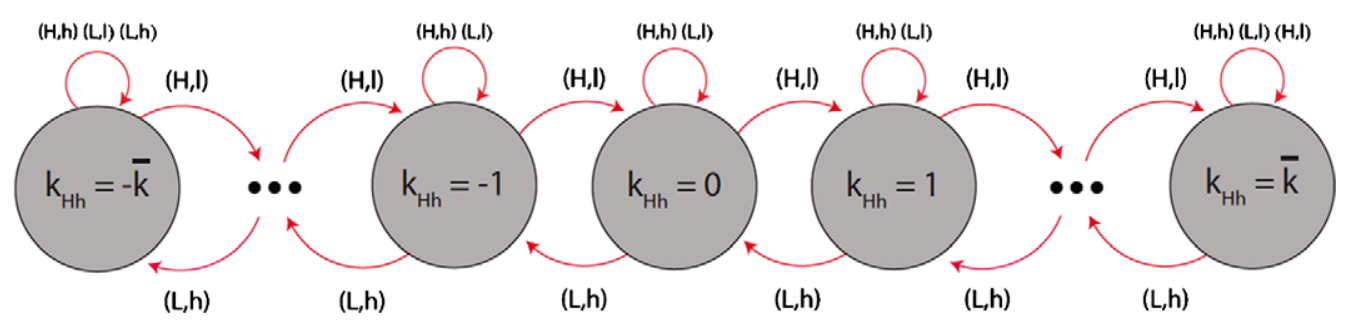

FIGURE 1. Structure of optimal and decentralized matching processes.

is, the queue can take the form of either $(k, 0,0, k)$ or $(0, k, k, 0)$, for some $k \geq 0$. The optimal dynamic mechanism is then identified by the maximal stock of $H$-squares (and $l$-rounds) and the maximal stock of $h$-rounds (and $L$-squares) that are kept waiting in queue. In the following proposition, we characterize the structure of the optimal mechanism. ${ }^{15}$

Proposition 1 (Optimal mechanisms). An optimal dynamic mechanism is identified by a pair of thresholds $\left(\bar{k}_{H}, \bar{k}_{h}\right) \in \mathbb{Z}_{+}$such that:

(i) if more than $\bar{k}_{H} H$-squares are present, excess $(H, l)$ pairs are matched immediately, and

(ii) if more than $\bar{k}_{h} h$-rounds are present, excess $(L, h)$ pairs are matched immediately.

As will soon be stated formally, the symmetry of our environment assures that, generically, an optimal mechanism corresponds to symmetric thresholds: $\bar{k} \equiv \bar{k}_{H}=\bar{k}_{h}$. A dynamic mechanism with symmetric thresholds $(\bar{k}, \bar{k})$ as defined in Proposition 1 is depicted in Figure 1, where $k_{H h} \equiv k_{H}-k_{h}$ captures the difference between the length of the queue of $H$-squares and the length of the queue of $h$-rounds. We call $k_{H h}$ the (signed) length of the $H$ - $h$ queue.

This process induces the following Markov chain. Let $k_{H h}^{t}$ denote the number of $H$ squares (or $l$-rounds) minus the number of $h$-rounds (or $L$-squares) at the end of time $t$, after the arrival of that period's square-round pair and any matches imposed by the mechanism. If an $(H, h)$ or an $(L, l)$ pair arrive in period $t+1$, the mechanism matches an $(H, h)$ or an $(L, l)$ pair immediately, and the state remains the same: $k_{H h}^{t}=k_{H h}^{t+1}$. Suppose an $(H, l)$ pair arrives in period $t+1$. As long as $0 \leq k_{H h}^{t}<\bar{k}$, the mechanism creates no matches and $k_{H h}^{t+1}$ becomes $k_{H h}^{t}+1$. If $k_{H h}^{t}<0$, the mechanism creates one $(H, h)$ match and one $(L, l)$ match, and $k_{H h}^{t+1}$ becomes $k_{H h}^{t}+1$. Finally, if $k_{H h}^{t}=\bar{k}$, the mechanism creates one $(H, l)$ pair, and $k_{H h}^{t+1}$ remains the same: $k_{H h}^{t+1}=k_{H h}^{t}=\bar{k}$. Analogous transitions occur with the arrival of an $(L, h)$ pair. Therefore, we can describe the

\footnotetext{
${ }^{15}$ In principle, there could be multiple mechanisms that are identified with the same thresholds. For instance, consider a mechanism in which an $(H, l)$ pair is matched whenever there are $\bar{k}_{H}+1$ or $\bar{k}_{H}+2$ $H$-squares present. Such a mechanism would be equivalent to a mechanism that matches $(H, l)$ pairs only when there are precisely $\bar{k}_{H}+1 H$-squares present. We focus only on the thresholds with the minimal magnitude, which identify outcomes fully, and ignore multiplicity that arises from prescriptions of the social planner over events that are never reached.
} 
probabilistic transition as follows. Denote by

$$
\mathbf{x}^{t} \equiv\left(x_{-\bar{k}}^{t}, x_{-\bar{k}+1}^{t}, \ldots, x_{\bar{k}-1}^{t}, x_{\bar{k}}^{t}\right)^{t r} \in\{0,1\}^{2 \bar{k}+1}
$$

the timed vector capturing the state, $x_{i}^{t}=\mathbf{1}\left(k_{H h}^{t}=i\right)$. That is, $x_{i}^{t}$ is an indicator that takes the value of 1 if the state is $i$ and 0 otherwise. Then

$$
\mathbf{x}^{t+1}=\mathbf{T}_{\bar{k}} \mathbf{x}^{t}
$$

where

$$
\mathbf{T}_{\bar{k}}=\left(\begin{array}{ccccc}
1-p(1-p) & p(1-p) & \ldots & 0 & 0 \\
p(1-p) & 1-2 p(1-p) & \ldots & 0 & 0 \\
0 & p(1-p) & \ldots & & \\
\vdots & \vdots & \ddots & \vdots & \vdots \\
0 & 0 & \ldots & p(1-p) & 0 \\
0 & 0 & \ldots & p(1-p) & 1-p(1-p)
\end{array}\right)
$$

This Markov chain is ergodic (i.e., irreducible, aperiodic, and positively recurrent). Therefore, an optimal mechanism corresponds to a matching process that reaches a steady state with a unique stationary distribution. For $T_{\bar{k}}$, the steady-state distribution is uniform so that each state $k_{H h}=-\bar{k}, \ldots, \bar{k}$ occurs with an equal probability of $\frac{1}{2 \bar{k}+1}$.

\subsection{Optimal thresholds}

In order to characterize the optimal threshold, we first evaluate the welfare corresponding to any arbitrary symmetric threshold. First, we compute the average total waiting costs incurred by agents waiting in line for one period of time. Since during the transition from time $t-1$ to time $t, 2\left|k_{H h}^{t-1}\right|$ agents wait in line, the total costs of waiting incurred during this one time period is $2\left|k_{H h}^{t-1}\right| c$. Thus, a mechanism with threshold $\bar{k}$ results in expected total costs of waiting equal to

$$
\frac{1}{2 \bar{k}+1}\left(\sum_{k_{H h}=-\bar{k}}^{\bar{k}} 2\left|k_{H h}\right|\right) c=\frac{2 \bar{k}(\bar{k}+1) c}{2 \bar{k}+1} .
$$

Next, we compute the average total surplus generated during one time period, tracking the Markov process described above. A newly-arrived pair is of type $(H, h)$ with probability $p^{2}$, in which case the optimal mechanism generates a surplus equal to $U_{H h}$. Similarly, when a new pair of type $(L, l)$ arrives, which occurs with probability $(1-p)^{2}$, the optimal mechanism generates a surplus equal to $U_{L l}$. Suppose an $(H, l)$ pair arrives at time $t$. If $k_{H h}^{t-1}<0$, the mechanism creates one $(H, h)$ pair and one $(L, l)$ pair, generating a surplus equal to $U_{H h}+U_{L l}$. If $0 \leq k_{H h}^{t-1}<\bar{k}$, the mechanism creates no matches (and no additional surplus), and if $k_{H h}^{t-1}=\bar{k}$, the mechanism creates one $(H, l)$ pair and generates a surplus equal to $U_{H l}$. Analogous conclusions pertain to the case in which 
an $(L, h)$ pair arrives. Thus, a mechanism with threshold $\bar{k}$ generates an expected total surplus equal to

$$
\begin{aligned}
& p^{2} U_{H h}+(1-p)^{2} U_{L l}+\frac{2 p(1-p)}{2 \bar{k}+1}\left[\bar{k}\left(U_{H h}+U_{L l}\right)+\frac{U_{H l}+U_{L h}}{2}\right] \\
& =p U_{H h}+(1-p) U_{L l}-\frac{p(1-p) U}{2 \bar{k}+1} .
\end{aligned}
$$

Therefore, the net expected total welfare per period, accounting for waiting costs, is

$$
p U_{H h}+(1-p) U_{L l}-\frac{p(1-p) U}{2 \bar{k}+1}-\frac{2 \bar{k}(\bar{k}+1) c}{2 \bar{k}+1} .
$$

The optimal threshold $\bar{k}^{\text {opt }}$ maximizes the welfare as given in (1). The following proposition summarizes our discussion and provides the full characterization of the optimal mechanism.

Proposition 2 (Optimal thresholds). The threshold

$$
\bar{k}^{\mathrm{opt}}=\left\lfloor\sqrt{\frac{p(1-p) U}{2 c}}\right\rfloor
$$

identifies an optimal dynamic mechanism. In this optimal mechanism, all available $(H, h)$ and $(L, l)$ pairs, and any number of $(H, l)$ or $(L, h)$ pairs exceeding $\bar{k}^{\mathrm{opt}}$, are matched immediately. Furthermore, the optimal mechanism is generically unique. ${ }^{16}$

The optimal threshold increases with the probability of any incongruent pair, $p(1-$ $p$ ), and with the degree of supermodularity $U$, which reflects the value of assortative matches. It decreases with waiting costs. In fact, when waiting costs are prohibitively high, namely when $c>\frac{p(1-p) U}{2}$, the maximal queue length is $\bar{k}^{\text {opt }}=0$ and all matches are instantaneous.

\subsection{Welfare}

We now turn to the expected per-period welfare in the steady state under the optimal mechanism. Were we to consider no costs of waiting, the optimal mechanism would naturally entail long waits to get the maximal possible match surplus asymptotically by matching only congruent pairs. We denote the resulting welfare by $S_{\infty} \equiv p U_{H h}+(1-$ p) $U_{L l}$.

The optimal threshold identified in Proposition 2 allows us to characterize the welfare achieved by the optimal mechanism through equation (1) and to get the following corollary.

Corollary 1 (Optimal welfare). The welfare under the optimal mechanism is given by $W^{\mathrm{opt}}(c)=S_{\infty}-\Theta(c)$, where $\Theta(c)$ is continuous, increasing, and concave in $c$, $\lim _{c \rightarrow 0} \Theta(c)=0$, and $\Theta(c)=p(1-p) U$ for all $c \geq \frac{p(1-p) U}{2}$.

\footnotetext{
${ }^{16}$ Multiplicity arises only when $\sqrt{\frac{p(1-p) U}{2 c}}$ is an integer.
} 
As waiting costs approach 0 , the welfare induced by the optimal mechanism approaches $S_{\infty}$. For costs large enough, the optimal mechanism matches all square-round pairs instantaneously as they arrive and the resulting welfare is $S_{\infty}-p(1-p) U$. For intermediate costs, the optimal mechanism generates welfare that is naturally in between these two values. ${ }^{17,18}$ The observation that welfare under the optimal mechanism decreases as $c$ increases is rather intuitive. Indeed, suppose $c_{1}>c_{2}$. Were we to implement the optimal mechanism with waiting $\operatorname{cost} c_{1}$ when the waiting $\operatorname{cost}$ is $c_{2}$, the distribution of matches would remain identical, while waiting costs would go down, thereby leading to greater welfare overall. Thus, the optimal mechanism generates greater welfare with waiting $\operatorname{cost} c_{2}$ than $c_{1}$. The amount by which welfare decreases when waiting costs increase depends on the number of agents expected to wait in line in the steady state. The higher the waiting costs, the lower the number of agents waiting in line on average. Therefore, the impact of an increase in costs by a fixed increment is greater at smaller costs, yielding the concavity of $\Theta(c) .{ }^{19}$

\section{Discretionary Matching}

Many dynamic matching processes are in essence discretionary, in the sense that participants have the choice of declining a match they do not wish to form: child adoption in the US and abroad, job searches in many industries, etc. It is therefore important to understand the implications of discretionary dynamics, particularly when considering centralized interventions. In this section, we provide a framework for analyzing a class of discretionary matching processes.

In our discretionary matching process, we assume individuals join the market in sequence and decide when to match with a potential partner immediately and when to stay in the market and wait for a potentially superior match. While the discretionary setting we study still requires some centralized governance, as matches occur according to some order, it provides a convenient benchmark for studying dynamic matching markets that are lightly regulated.

We assume that at each period $t$ there are three stages. First, a square and round enter the market with random attributes as before: with probability $p$ the square is an $H$-square and with probability $p$ the round is an $h$-round. Second, individuals of each type are ordered by some priority rule that we describe formally below. In the third stage, each square and round declare their demands-whether a square will match only with an $h$-round, or is willing to match with either an $h$-round or an $l$-round, and whether a round will match only with an $H$-square, or with either an $H$-square or an $L$-square.

\footnotetext{
${ }^{17}$ The value of $S_{\infty}$ is effectively the analogue of the value generated by an "omniscient" planner in our setting, which is used as one benchmark in Akbarpour et al. (2020). Corollary 1 suggests that the omniscient planner's value is a valid feasible benchmark when waiting costs vanish.

${ }^{18}$ In the Appendix, we provide the analytical formula for $\Theta(c)$ in terms of the fundamental parameters of our setting. In fact, simple algebraic manipulations imply that

$$
S_{\infty}-\sqrt{2 p(1-p) U c}-c \leq W^{\mathrm{opt}}(c) \leq S_{\infty}-\sqrt{2 p(1-p) U c}+c .
$$

${ }^{19}$ Continuity follows directly from concavity. Alternatively, fix any mechanism that is optimal for some waiting costs. An increase in waiting costs reduces the resulting welfare continuously, in fact linearly.
} 
Given the order and the participants' demands, the market clears sequentially according to the priority rule. Any remaining participants proceed to period $t+1$ at the additional cost of $c$.

\subsection{The matching process}

In each period $t$, one square $w^{t}$ and one round $r^{t}$ arrive at the market, and their types are realized. Upon their arrival, a period- $t$ stage-game begins:

$$
G^{t} \equiv\left\{I^{t},\left(D_{i}\right)_{i \in I^{t}}, \phi,\left(u_{i}(\cdot ; \phi): \prod_{i \in I^{t}} D_{i} \rightarrow \mathbb{R}\right)_{i \in I^{t}}\right\} .
$$

The components of $G^{t}$ are defined as follows. The set of players is $I^{t} \equiv H^{t} \cup L^{t} \cup h^{t} \cup l^{t}$, where $H^{t} \subseteq\left\{x^{t^{\prime}}: 1 \leq t^{\prime} \leq t\right\}$ is the set of $H$-squares present in the market in period $t$, and the other sets, $L^{t}, h^{t}$, and $l^{t}$, are defined similarly. Each $H$-square, say player $i$, in $I^{t}$ chooses an action in $D_{i}=\{h, l\}$, with $h$ denoting a demand for only $h$-rounds and $l$ denoting a demand for either type of round. ${ }^{20}$ Action sets for other agents' types are defined analogously. A priority rule $\phi$ assigns a linear order over each set $H^{t}, L^{t}, h^{t}$, and $l^{t}$. First, we consider a first-in-first-out (FIFO) protocol, which assigns a linear order $\succ$ over, say, $H^{t}$ such that

$$
\forall x^{t^{\prime}}, x^{t^{\prime \prime}} \in H^{t}, \quad x^{t^{\prime}} \succ x^{t^{\prime \prime}} \Longleftrightarrow t^{\prime}<t^{\prime \prime} \leq t .
$$

There is anecdotal evidence that order of arrivals affects the order of matches in many markets, and FIFO is a commonly used protocol. For instance, in the child adoption context, many countries follow a FIFO protocol to match relinquished children to adoptive parents. ${ }^{21}$ In Section 6, we discuss alternative priority protocols.

The stage-game payoffs are determined by sequential market clearing. First, we take $H$-squares and $h$-rounds in the order induced by $\phi$ and form as many $(H, h)$ pairs as possible (regardless of their demands). ${ }^{22}$ If there are remaining $H$-squares demanding $l$ rounds, they are matched with $l$-rounds sequentially according to $\phi$ and independently of the demands made by the $l$-rounds. $h$-rounds demanding $L$-squares are matched analogously. All remaining $L$-squares and $l$-rounds who are flexible in their demands form matches sequentially in the order induced by $\phi$. The stage-game payoff for a type$x$ agent matched with a type- $y$ agent is $U_{x}(y)$. If a player remains unmatched, her stagegame payoff is $-c$.

We complete the definition of a dynamic discretionary matching game by characterizing the evolution of the stage games $G^{t}$, and each player's dynamic-game payoff.

\footnotetext{
${ }^{20}$ This restriction on the action space is made for simplicity of exposition. An equilibrium similar to the one we describe below arises if we allow players to demand only inferior matches on the other side of the market, or to submit a demand for no one at all.

${ }^{21}$ For example, see the protocol adopted by the China Center of Children's Welfare and Adoption (CCCWA) here: http://www.aacadoption.com/programs/china-program.html.

${ }^{22}$ This market-clearing assumption allows us to simplify some steps of the proofs, and avoid inefficient equilibria in which $(H, h)$ pairs remain on the market unmatched.
} 
The initial set of players is $I^{0} \equiv \varnothing$. All players in $I^{t}$ who remain unmatched in period $t$, together with new arrivals, form $I^{t+1}$. Consider a player $i$, who arrives in period $t$ and is matched at $t^{\prime \prime} \in \mathbb{Z}_{+} \cup\{\infty\}$. Such a player receives stage-game payoffs $\left(u_{i}^{t}, u_{i}^{t+1}, \ldots\right)$, and a dynamic-game payoff $\sum_{t^{\prime}=t}^{\infty} u_{i}^{t^{\prime}}(\in \mathbb{R} \cup\{-\infty\})$, where $u_{i}^{t^{\prime \prime}}$ is $i$ 's match utility, $u_{i}^{t^{\prime}}=0$ for $t^{\prime}>t^{\prime \prime}$, and $u_{i}^{t^{\prime}}=-c$ for any $t^{\prime}<t^{\prime \prime}$.

The dynamic game has complete information and arbitrary (dynamic) strategies. Each player $i$, say an $H$-square, chooses a demand every period she remains in the market, since her arrival until she matches. A (dynamic) strategy $\sigma_{i}$ indicates the probability of demanding an $h$-round in each of these periods and can depend on the complete history from $t=0$. As before, let $\mathbf{s}^{t}=\left(s_{H}^{t}, s_{L}^{t}, s_{h}^{t}, s_{l}^{t}\right)$ be the state (or stock) at period $t$, and let $q_{i}^{t} \in \mathbb{Z}_{+}$be player $i$ 's rank according to $\phi$ in period $t .{ }^{23}$ Let $\theta_{i}^{t} \equiv\left(\mathbf{s}^{t}, q_{i}\right)$ denote an augmented state for player $i$.

Definition 1. A strategy $\sigma_{i}$ is a stationary and deterministic strategy (SD-strategy) for an $H$-square $i$ if there exists $\psi_{i}^{H}:\left\{(s, q) \in Z_{+}^{5}\right\} \rightarrow\{h, l\}$ such that, for any $t$ such that $i \in H^{t}$ and $\theta_{i}^{t}=\left(s^{t}, q_{i}^{t}\right)$, player $i$ demands $\psi_{i}^{H}\left(s^{t}, q_{i}^{t}\right)$.

We similarly define SD-strategies for $L$-squares, $h$-rounds, and $l$-rounds. A symmetric, stationary, and deterministic strategy profile, which we name stationary* strategy profile, is a profile of SD-strategies, such that all players of the same type use the same strategy, i.e., $\psi_{i}^{x}=\psi^{x}$ for all $t, i \in x^{t}$, and $x=H, L, h, l$. We denote a stationary* strategy profile by $\Psi=\left(\psi^{H}, \psi^{L}, \psi^{h}, \psi^{l}\right)$.

Definition 2. A stationary* strategy-profile $\Psi$ is a stationary* equilibrium if it is an equilibrium of the dynamic matching game. ${ }^{24}$

For simplicity, we assume a symmetric setting (results pertaining to asymmetric settings appear in Section 7):

$$
U_{H}(h)-U_{H}(l)=U_{h}(H)-U_{h}(L) \quad \text { and } \quad U_{L}(h)-U_{L}(l)=U_{l}(H)-U_{l}(L),
$$

and that the environment is regular: $p\left(U_{H}(h)-U_{H}(l)\right) \neq k c$ for all natural numbers $k \in \mathbb{N}$. Regularity assures that neither squares nor rounds are ever indifferent between waiting in queue and matching immediately with an available partner. ${ }^{25}$

\subsection{Equilibrium characterization with the FIFO protocol}

In this section, we present necessary conditions for a stationary* equilibrium that are sufficient to compute equilibrium welfare. We guarantee the existence of stationary* equilibria and provide their characterization in the Appendix.

\footnotetext{
${ }^{23}$ That is, under FIFO, $q_{i}^{t}=1$ if player $i$ arrived before all other $H$-squares in $H^{t}, q_{i}^{t}=2$ if player $i$ arrived second among all other $H$-squares in $H^{t}$, and so on.

${ }^{24}$ In a stationary* equilibrium, we allow a player's deviation to be any dynamic strategy, including history dependent and random.

${ }^{25}$ The assumption of regularity simplifies presentation but is not crucial. Similar analysis follows without it for any arbitrary tie-breaking rule.
} 
By construction, at the beginning of a period, the queue cannot entail both $\mathrm{H}$ squares and $h$-rounds. As before, we denote the (signed) length of the $H$ - $h$ queue after an arrival of a new pair by $s_{H h} \equiv s_{H}-s_{h}$, and after agents form matches by $k_{H h} \equiv k_{H}-k_{h}$. We first consider the decisions of $H$-squares (analogous analysis holds for $h$-rounds). Suppose an $H$-square arrives at the market and an $h$-round is available, one that had either been waiting in the queue or one that has just arrived as well. In this case, an $H$-square is matched immediately to an $h$-round, the identities of whom are prescribed by the order of arrival. In particular, if the arriving $H$-square is the first in line, that square is matched to an $h$-round. If there are $H$-squares already in queue, the available $h$-round must have arrived with our $H$-square, and is matched with the first $H$-square in the queue. The newly arrived $H$-square then has a choice between waiting in line and matching with an $l$-round. However, this square's decision is equivalent to that of the last $H$-square who arrived and decided to wait. Therefore, in a stationary* equilibrium, the new $H$-square waits and the queues remain as they were.

Suppose now that an $H$-square enters the market and no $h$-round is available. Then there is at least one $l$-round available. Thus, the $H$-square has to decide whether to match immediately with an $l$-round or to wait, based on the number of $H$-squares already waiting. An immediate match with an $l$-round delivers $U_{H}(l)$, whereas waiting in line until eventually matching with an $h$-round delivers $U_{H}(h)$ at an uncertain waiting cost.

Note that if an $H$-square decides to wait in the queue, she will wait until matching with an $h$-round, rather than match with an $l$-round at a later point. Indeed, as matches form on a FIFO basis, the $H$-square's position in the queue moves up over time, and the expected time until matching with an $h$-round becomes shorter. The expected waiting time until a match with an $h$-round is therefore solely determined by the number of other $H$-squares who precede her in the queue. The following result identifies bounds on the size of the $H$ - $h$ queue.

Lemma 1 (FIFO thresholds). In all stationary* equilibria under FIFO, in all periods, $-\bar{k}^{\text {fifo }} \leq k_{H h} \leq \bar{k}^{\text {fifo }}$, where ${ }^{26}$

$$
\bar{k}^{\text {fifo }} \equiv\left\lfloor\frac{p\left(U_{H}(h)-U_{H}(l)\right)}{c}\right\rfloor=\left\lfloor\frac{p\left(U_{h}(H)-U_{h}(L)\right)}{c}\right\rfloor .
$$

Intuitively, the time until an $h$-round enters the market is distributed geometrically (with parameter $p$ ), so the expected time until an $h$-round arrives at the market is $\frac{1}{p}$. An $H$-square who is $k$ th in line in the queue will be matched when the $k$ th $h$-round arrives, which is expected to occur in $\frac{k}{p}$ periods. The expected waiting costs are therefore $\frac{k c}{p}$, which generate an increase in match utility of $U_{H}(h)-U_{H}(l)$ (relative to matching with an $l$-round immediately). An $H$-square will wait as long as the expected benefit of waiting exceeds its costs, i.e., whenever $\frac{k c}{p}<U_{H}(h)-U_{H}(l)$, which is the comparison underlying the maximal size of the queue described in Lemma 1. Our regularity assumption

\footnotetext{
${ }^{26}$ In the Appendix, we show that in all stationary* equilibria the full support of the $H$ - $h$ queue is $\left\{-\bar{k}^{\text {fifo }}, \ldots, \bar{k}^{\text {fifo }}\right\}$. Therefore, the bounds described in this lemma are achieved in equilibrium.
} 
further guarantees that an $H$-square or an $h$-round are never indifferent between waiting in line and matching immediately. Whenever there are fewer than $\bar{k}^{\text {fifo }} H$-squares in the queue, a new $H$-square will wait in the market. Whenever there are $\bar{k}^{\text {fifo }}$ or more $H$-squares in the queue, the new $H$-square prefers to match with an $l$-round immediately. An analogous description holds for $h$-rounds and our symmetry assumptions assure that the maximal queue length is identical for $H$-squares and $h$-rounds.

We now turn to the decisions of $L$-squares and $l$-rounds. An $l$-round (similarly, an $L$-square) may decide to wait, hoping to match with an $H$-square who will become available when the line for $H$-squares exceeds $\bar{k}^{\text {fifo }}$. In principle, there are two effects at work. The first is similar to that experienced by the $H$-squares waiting in line: the longer the queue of $l$-rounds already waiting, the longer a new $l$-round has to wait. The second effect is due to $H$-squares' equilibrium behavior: the longer is the queue, the closer $H$ squares are to the threshold $\bar{k}^{\text {fifo }}$ and to the point of accepting matches with $l$-rounds. As it turns out, at least the last $l$-round in the queue has an incentive to match immediately with any square. Intuitively, consider the first $l$-round, say player $i$, arriving at the market. There cannot be other $H$-squares waiting in the market since any such squares would have arrived with $l$-rounds, contradicting our $l$-round being first in line. Suppose player $i$ arrives with an $L$-square. By Lemma 1, the first $\bar{k}^{\text {fifo }} H$-squares wait in line until they are matched with an $h$-round. Thus, player $i$ has to wait for the arrival of at least $\bar{k}^{\text {fifo }}+1 H$-squares to match with an $H$-square. This wait is too long for agent $i$ to justify turning down a match with an $L$-square. Indeed, the expected cost of waiting until the $\left(\bar{k}^{\text {fifo }}+1\right)$-th arriving $H$-square is $\frac{\left(\bar{k}^{\text {fifo }}+1\right) c}{p}$, which is strictly greater than the benefit from waiting since

$$
U_{l}(H)-U_{l}(L)<U_{h}(H)-U_{h}(L)=U_{H}(h)-U_{H}(l)<\frac{\left(\bar{k}^{\text {fifo }}+1\right) c}{p},
$$

where the last inequality follows from the definition of $\bar{k}^{\text {fifo }}$. It follows that our player $i$ would therefore prefer to match with an $L$-square, who is available, immediately. In fact, this intuition generalizes and yields the following result.

Lemma 2 (Equilibrium under FIFO). There exists a stationary* equilibrium such that there are never both an L-square and an l-round waiting in the market.

Lemma 2 implies that there exists a stationary* equilibrium that follows a protocol similar to that implemented by the optimal mechanism, though the threshold governing when incongruent matches are formed, $\bar{k}^{\text {fifo }}$, may differ from the optimal threshold $\bar{k}^{\text {opt }}$. Lemma 1 and its discussion in the Appendix guarantee that, since the behavior of $\mathrm{H}$ squares and $h$-rounds is the same in all stationary* equilibria, so is the welfare generated by matches involving $H$-squares and $h$-rounds. Therefore, the stationary* equilibrium described by Lemma 2 , in which $L$-squares and $l$-rounds do not delay matching with one another, is the one that maximizes welfare, as stated in the following corollary.

Corollary 2. The stationary* equilibrium in which there are never both an L-square and an l-round waiting in the market is welfare-maximizing among all stationary* equilibria under FIFO. 


\subsection{Steady state of discretionary matching}

As for the optimal mechanism, the length of the $H$ - $h$ queue $k_{H h}$ in the equilibrium described in Lemma 2 and Corollary 2 is characterized by a Markov chain with a transition matrix analogous to that described in Section 3.2. Similar analysis allows the characterization of the equilibrium steady state of the discretionary process under the FIFO protocol.

Proposition 3 (Discretionary steady state). The welfare-maximizing stationary* equilibrium under FIFO is associated with a unique steady-state distribution over queue lengths, such that the length of the $H$-h queue $k_{H h}=k_{H}-k_{h}$ is uniformly distributed over $\left\{-\bar{k}^{\text {fifo }}, \ldots, \bar{k}^{\text {fifo }}\right\}$ and, in any period, the queues contain only $H$-squares and l-rounds or only h-rounds and L-squares.

The threshold $\bar{k}^{\text {fifo }}$ is determined by the decisions of $H$-squares and $h$-rounds to wait, as specified in Lemma 1. The crucial difference between the discretionary and optimal mechanism is the threshold placed on the maximal stock of $H$-squares or $h$ rounds waiting. A decision to wait in the market by, say, a square imposes a negative externality on succeeding squares, as it potentially affects their waiting time, and possibly the quality of their matches ${ }^{27}$ as well as on the round she would otherwise match with. However, a decision to wait can also impose a positive externality on future desirable agents on the other side of the market, who would find a ready desirable agent upon arrival.

To glean some intuition for the relative strength of these externalities, consider the extreme case in which, in the discretionary process, matches are immediate $\left(\bar{k}^{\text {fifo }}=0\right)$, which happens when $\frac{c}{p}>U_{H}(h)-U_{H}(l)$. In such a market, consider an $(H, l)$ pair arriving when no other agents are present. In the discretionary setting, the pair would match immediately. Might a social planner want to keep this pair waiting? Suppose the planner holds on to the $(H, l)$ pair until an $h$-round arrives with a square of either type. At that point, the $H$-square is to be matched with the $h$-round, while the $l$-round is to be matched with the newly-arrived square. To simplify our illustration, suppose that all other participants are matched instantaneously. The $H$-square certainly does not benefit from this imposed wait (else, she would wait even in the discretionary setting). The cost imposed on the $l$-round entailed by waiting for an $h$-round is $c / p$. Now, the anticipated $h$-round may arrive with either an $H$-square, with probability $p$, or with an $L$ square, with probability $1-p$. In the latter case, the positive externality of our $H$-square on this $h$-round comes to light-the $h$-round matches with an $H$-square instead of an $L$ square he would match with otherwise, generating a marginal benefit of $U_{H}(h)-U_{H}(l)$ (since match payoffs are symmetric across market sides). This positive externality is overwhelmed by the cost of waiting incurred by the $l$-round, even ignoring all other negative externalities on the match qualities of the original $l$-round as well as the square

\footnotetext{
${ }^{27}$ From a welfare perspective, the externality on the quality of the match is of less importance. As long as a social planner views identical agents as interchangeable, an immediate mismatch or a later mismatch have similar welfare consequences.
} 
arriving with the $h$-round, since $\frac{c}{p}>U_{H}(h)-U_{H}(l)>(1-p)\left(U_{H}(h)-U_{H}(l)\right)$. In particular, delaying a match is suboptimal from the social planner's perspective. This intuition extends-the negative externalities dominate and the optimal mechanism is always governed by a smaller threshold for waiting than the one selected through equilibrium in the discretionary process.

Corollary 3 (Thresholds' comparison). Maximal waiting queues are longer under FIFO than they are under the optimal mechanism. That is, $\bar{k}^{\mathrm{opt}} \leq \bar{k}^{\mathrm{fifo}}$, with strict inequality for sufficiently small waiting costs $c$.

\subsection{Welfare}

Since the protocols are similar except for the queues' thresholds, the expected perperiod welfare in the steady state characterized in Proposition 3 can be found using an analogous derivation to that carried out for the optimal mechanism. This derivation leads to an expression mirroring equation (1), accounting for the discretionary process' threshold $\bar{k}^{\text {fifo }}$. Namely, the expected per-period net welfare is given by

$$
W^{\text {fifo }}(c)=S_{\infty}-\frac{p(1-p) U}{2 \bar{k}^{\text {fifo }}+1}-\frac{2 \bar{k}^{\text {fifo }}\left(\bar{k}^{\text {fifo }}+1\right) c}{2 \bar{k}^{\text {fifo }}+1},
$$

where $\bar{k}^{\text {fifo }}$ is defined in (2). To summarize, we have the following corollary.

Corollary 4 (Decentralized welfare). The maximum equilibrium welfare under FIFO is given by $W^{\text {fifo }}(c)=S_{\infty}-\Psi(c)$, where $\lim _{c \rightarrow 0} \Psi(c)=p\left(U_{H}(h)-U_{H}(l)\right)$, and $\Psi(c)=$ $p(1-p) U$ for all $c \geq p\left(U_{H}(h)-U_{H}(l)\right)$.

Recall Corollary 1, which characterized the welfare under the optimal mechanism. By definition, the welfare generated under the optimal mechanism is higher than that generated by the discretionary process, so that $\Theta(c) \leq \Psi(c)$ for all $c$. While the optimal mechanism generates welfare that is decreasing in waiting costs, this is not necessarily the case under the discretionary process. Furthermore, while the welfare under the optimal mechanism approaches $S_{\infty}$ as waiting costs diminish, this is not the case under the discretionary process. As waiting costs become very small, there is a race between two forces. For any given threshold, the overall waiting costs decline. However, in equilibrium, discretionary thresholds increase, leading to greater expected wait times. As it turns out, the balance between these two forces generates significant welfare losses, given by $p\left(U_{H}(h)-U_{H}(l)\right)$, even for vanishing costs.

\section{WELFARE COMPARISONS}

By construction, the optimal mechanism generates welfare that is at least as high as that generated by the discretionary process. ${ }^{28}$ In this section, we inspect how the welfare wedge responds to the underlying parameters of the environment, suggesting the

\footnotetext{
${ }^{28}$ It is possible to show that the optimal mechanism generally presents a Pareto improvement with respect to the decentralized setting. In fact, it is easy to see that $L$-squares and $l$-rounds are better off under
} 
settings in which centralized intervention might be particularly useful. The following proposition captures the effects on the welfare wedge $W^{\text {opt }}(c)-W^{\text {fifo }}(c)$ of the waiting costs $c$, the frequency $p$ of $H$-squares or $h$-rounds, and the utility benefit of desirable types from matching with desirable types relative to less desirable ones.

Proposition 4 (Welfare wedge-comparative statics). (i) For any interval $[\underline{c}, \bar{c}$ ), where $\underline{c}>0$, there is a partition $\left\{\left[c_{i}, c_{i+1}\right)\right\}_{i=1}^{M-1}$, where $\underline{c}=c_{1}<c_{2}<\cdots<c_{M}=\bar{c}$, such that $W^{\mathrm{opt}}(c)-W^{\mathrm{fifo}}(c)$ is continuous and increasing over $\left(c_{i}, c_{i+1}\right)$ and

$$
W^{\text {opt }}\left(c_{i}\right)-W^{\text {fifo }}\left(c_{i}\right)>W^{\text {opt }}\left(c_{i+1}\right)-W^{\text {fifo }}\left(c_{i+1}\right)
$$

for all $i=1, \ldots, M-1$.

(ii) As c becomes vanishingly small, the welfare gap $W^{\mathrm{opt}}(c)-W^{\mathrm{fifo}}(c)$ converges to a value that is increasing in $p \in(0,1)$ and in $U_{H}(h)-U_{H}(l)$.

To see the intuition for the comparative statics corresponding to waiting costs, notice that an increase in costs has two effects on the welfare gap. Since the equilibrium threshold under the discretionary process is greater than the optimal threshold (Corollary 3), an increase in waiting costs has a direct effect of magnifying the welfare gap. Nonetheless, there is also an indirect effect of an increase in waiting costs that arises from the potential changes in the induced thresholds. Consider a slight increase in waiting costs such that the optimal threshold does not change, but the discretionary threshold decreases. The discretionary process is then "closer" to the optimal process-both the matching surplus and the waiting costs are closer and the welfare gap decreases. In fact, as costs become prohibitively high, both processes lead to instantaneous matches and identical welfare levels. As we show in the proof of Proposition 4, the indirect effect overwhelms the direct effect at precisely such transition points and acts to shrink the welfare gap. The construction of the partition is done as follows. Each atom $\left[c_{i}, c_{i+1}\right)$ of the partition corresponds to constant thresholds under the discretionary process. Over these intervals, only the direct effect operates and the welfare gap is increasing. Each of the endpoints $\left\{c_{i}\right\}_{i}$ corresponds to a decrease of the discretionary threshold by one. Therefore, when comparing two such endpoints, the indirect effect kicks in and the decreasing trend of the welfare gap emerges. Figure 2 depicts the resulting pattern the welfare gap exhibits for $U_{H}(h)=U_{h}(H)=3, U_{H}(l)=U_{h}(L)=U_{L}(h)=U_{l}(H)=1$, $U_{L}(l)=U_{l}(L)=0$, and $p=0.3$. As suggested by the proposition, $W^{\text {opt }}(c)-W^{\text {fifo }}(c)$ is piecewise increasing in $c$. Nevertheless, overall, the gap has a decreasing trend.

To glean some intuition on the comparative statics the welfare gap displays with respect to $p$, consider two type distributions governed by $p_{1}$ and $p_{2}$ such that $p_{1}<p_{2}=$ $m p_{1}, m>1$. The individual incentives to wait for $H$-squares and $h$-rounds are higher under $p_{2}$ than under $p_{1}$. In fact, in the discretionary setting, the distribution of steadystate queue length is the uniform distribution where, from (2), under $p_{2}$, roughly $1-1 / m$

the optimal mechanism (as it implies better matches and shorter waiting times). Moreover, one can show that, as long as $\bar{k}^{\text {opt }} \geq 2$, the expected payoff of $H$-squares and $h$-rounds as they enter the market is higher under the optimal mechanism than under the discretionary process as well. 


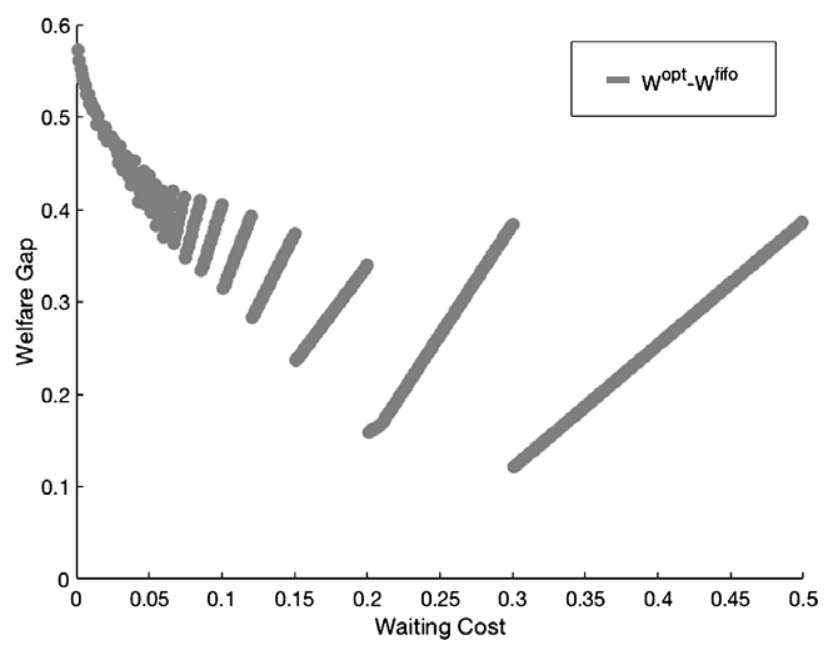

FIGURE 2. Welfare gap between optimal and discretionary matching (FIFO) as a function of costs.

of the probability mass is allocated to queue lengths larger than those realized under $p_{1}$. For each of these large steady-state queue lengths, we have more pairs of agents waiting, i.e., increased per-period waiting costs. The optimal mechanism internalizes the negative externalities, so the effect of the increased waiting costs is weaker. On the other hand, the benefit of this increase in queue length is a lower chance of producing mismatches. However, for sufficiently low $c$, the match surplus under $p_{1}$ is already close to its optimum of $S_{\infty}$ and this effect is weak; in particular, the difference in terms of match surplus that the optimal and discretionary processes generate is similar under $p_{1}$ and $p_{2}$. Therefore, for sufficiently low $c$, the dominant effect is the one produced by the difference in expected waiting costs, which generates our comparative statics. ${ }^{29}$ Note that as $p$ approaches 0 or 1 , both the optimal mechanism and the discretionary processes generate similar welfare levels as in those cases incongruent pairs arrive at a vanishing rate. The intuition for the comparative statics pertaining to $U_{H}(h)-U_{H}(l)$ follows identical lines.

Going back to our assumption of supermodular preferences, the construction of the optimal mechanism would remain essentially identical were preferences submodular (with an appropriate relabeling of market participants). However, in the discretionary setting, submodular preferences would lead to a negative welfare effect compounding the negative externalities present in our setup. Namely, individual incentives would be misaligned with market-wide ones. In that respect, our comparison of optimal and discretionary processes assuming supermodular preferences is a conservative one.

Similarly, considering waiting costs that differ across the two sides of the market would lead to a greater welfare wedge as well. Intuitively, suppose that squares experience a waiting cost of $c_{S}$ and rounds experience a waiting cost of $c_{R}$, where $c_{S}>c_{R}$,

\footnotetext{
${ }^{29}$ In fact, we can show that for any $\Delta p>0$, there exists $\delta>0$ such that for every $c<\delta$ and $p \in[0,1-\Delta p]$, the welfare wedge under $p+\Delta p$ and $c$ is greater than under $p$ and $c$. Furthermore, $\delta \rightarrow 0$ as $\Delta p \rightarrow 0$.
} 
with an average cost of $c=\left(c_{S}+c_{R}\right) / 2$. The optimal mechanism with asymmetric costs would coincide with that corresponding to identical costs of $c$ since per-pair costs are the same in both cases. In the discretionary process, $H$-squares would be willing to wait when the queue of $H$-squares is no longer than $\bar{k}_{S}^{\text {fifo }}$ and $h$-rounds would be willing to wait when the queue of $h$-rounds is no longer than $\bar{k}_{R}^{\text {fifo }}$, where $\bar{k}_{S}^{\text {fifo }}=\left\lfloor\frac{p\left(U_{H}(h)-U_{H}(l)\right)}{c_{S}}\right\rfloor$ and $\bar{k}_{R}^{\text {fifo }}=\left\lfloor\frac{p\left(U_{h}(H)-U_{h}(L)\right)}{c_{R}}\right\rfloor$. Suppose $\frac{p\left(U_{H}(h)-U_{H}(l)\right)}{c_{x}} \in \mathbb{N}$ for $x=S, R$ to avoid rounding issues. From convexity, it follows that the threshold $\bar{k}^{\text {fifo }}$ corresponding to identical costs of $c$ satisfies $\bar{k}^{\text {fifo }} \leq\left(\bar{k}_{S}^{\text {fifo }}+\bar{k}_{R}^{\text {fifo }}\right) / 2$. Therefore, the excessive waiting discretionary processes exhibit would be even more pronounced when costs are asymmetric across market sides.

\section{Alternative protocols}

So far, we have shown that intervention in dynamic matching markets can have a substantial impact on welfare, at least when centralization utilizes the optimal dynamic mechanism. Nonetheless, the full-fledged optimal mechanism may be hard to implement. It requires that the formation of matches, even those of individuals who would prefer to wait in the market, be within the purview of the centralized planner. It also requires the central planner to monitor the market continuously to determine when matches should be formed, which may be administratively costly. Improvements to discretionary settings under FIFO can be achieved by mechanisms that relax one of these two requirements. To address the first issue, one can consider a discretionary setting in which per-period taxes are introduced for the agents that decide to wait. Our characterization of the optimal mechanism allows the identification of a budget-balanced tax scheme that implements the optimal welfare levels without distorting agents' incentives to enter the market to begin with. To address the second issue, one can consider an alternative protocol in which the centralized clearinghouse matches all available agents every fixed number of periods. Details of these alternative protocols are available in the working-paper version (Baccara et al. 2018).

In this section, we analyze an alternative setting that does not require continuous market monitoring and can provide substantial welfare improvements over the discretionary matching process under FIFO. While the FIFO protocol we analyze resembles discretionary processes in various applications, it generates excessive waiting. It is then natural to consider alternative protocols in which waiting is disciplined. We consider here the often discussed last-in-first-out (LIFO) protocol (see, e.g., Hassin 1985, and more recently, Platz and Østerdal 2017, as well as references therein). Under the LIFO protocol, waiting is disciplined as it entails a transition to a bad position in the queue and, consequently, may improve on the welfare generated by the FIFO protocol. It is important to keep in mind, however, that protocols such as LIFO face well-known implementation hurdles. ${ }^{30}$

\footnotetext{
${ }^{30}$ In particular, they are subject to manipulation as they introduce incentives to leave and reenter queues (see Margaria 2019). They are also considered "unfair" in that individuals who exert no cost of waiting are catered to first, while identical others who have been waiting remain in the queue.
} 
Formally, we study a discretionary setting that has the same structure described in Section 4.1 but, once every agent on the market has specified their demands, matches form according to a LIFO protocol. This protocol assigns a linear order $\succ$ over, say, $H^{t}$ such that $\forall x^{t^{\prime}}, x^{t^{\prime \prime}} \in H^{t}, x^{t^{\prime \prime}} \succ x^{t^{\prime}} \Longleftrightarrow t^{\prime}<t^{\prime \prime} \leq t$.

We first consider the decisions of $H$-squares (and omit the analogous discussion for $h$-rounds). If an $H$-square finds an $h$-round upon arrival, the $H$-square matches with the last arrived $h$-round. If no $h$-round is available, the $H$-square needs to decide whether to match with the last $l$-round, who must have just arrived together with the $H$-square, or to wait in the queue. Under LIFO, this decision is independent of other $H$-squares who have been waiting in the queue. Rather, the decision depends on the anticipated behavior of $H$-squares who will arrive at the market in future periods. We consider an SD-strategy $\psi_{H}$ for $H$-squares relying on a threshold $\bar{k}_{H}$. If no $h$-round is available, an $H$-square, say player $i$, waits by demanding an $h$-round as long as her rank $q_{i}$ according to LIFO is at most $\bar{k}_{H}$. To gain intuition for our equilibrium characterization, suppose that all $H$-squares, including player $i$, use the threshold $\bar{k}_{H}=1$. If player $i$ finds no available $h$-round upon arrival, then she waits by demanding an $h$-round. In the next period, player $i$ continues to wait if either a pair $(H, h)$ or a pair $(L, l)$ arrive, since in these scenarios her rank according to LIFO remains the same. However, if an $(H, l)$ pair arrives, the new $H$-square, who also uses $\bar{k}_{H}=1$, demands an $h$-round. According to $\psi_{H}$, player $i$ then demands an $l$-round and leaves the market. Finally, if an $(L, h)$ pair arrives, player $i$ matches to the $h$-round. To summarize, player $i$ exits the market matched with either an $h$-round or an $l$-round, with probability $1 / 2$ each, as soon as the first incongruent pair arrives. Since the expected number of periods until the first arrival of an incongruent pair is $\frac{1}{2 p(1-p)}$, the expected payoff for player $i$ is

$$
\frac{U_{H}(h)+U_{H}(l)}{2}-\frac{c}{2 p(1-p)}
$$

Consider a possible deviation of player $i$ in which $i$ demands an $l$-round when she finds no available $h$-rounds upon her arrival. This deviation is not strictly profitable if and only if

$$
U_{H}(l) \leq \frac{U_{H}(h)+U_{H}(l)}{2}-\frac{c}{2 p(1-p)},
$$

which we can rewrite as

$$
\frac{p(1-p)\left(U_{H}(h)-U_{H}(l)\right)}{c} \geq 1=\frac{\bar{k}_{H}\left(\bar{k}_{H}+1\right)}{2} .
$$

Consider another potential deviation by player $i$ : if one more $H$-square arrives after player $i$ and no $h$-round is available, player $i$, instead of demanding an $l$-round, increases her threshold to $\bar{k}_{H}^{\prime}=2$ indefinitely and remains in the market. If player $i$ uses the threshold $\bar{k}_{H}^{\prime}=2$, while all other $H$-squares use $\bar{k}_{H}=1$, player $i$ will match to an $h$-round for sure. We use an absorbing Markov chain to compute the expected continuation payoff for player $i$. In what follows, we normalize time to event time, denoted by $\tau$, which increases upon each arrival of an incongruent pair (which, in expectation, occurs 
every $1 / 2 p(1-p)$ periods). The state space is $\{1,2, h\}$ : the two transient states ( 1 and 2$)$ denote the $H$-square's rank, and the absorbing state ( $h$ ) denotes player $i$ matching an $h$-round. The matrix of transition probabilities $p_{i j}$ from state $i$ to state $j$ is

$$
P=\left[\begin{array}{cc}
Q & R \\
\mathbf{0} & 1
\end{array}\right], \quad \text { where } Q=\left[\begin{array}{cc}
0 & 1 / 2 \\
1 / 2 & 1 / 2
\end{array}\right] \text { and } R=\left[\begin{array}{c}
1 / 2 \\
0
\end{array}\right]
$$

The matrix $Q$ represents transition probabilities between transient states. ${ }^{31}$ Let

$$
T \equiv\left(I_{2}-Q\right)^{-1} \cdot 1=4\left[\begin{array}{cc}
1 / 2 & 1 / 2 \\
1 / 2 & 1
\end{array}\right]\left[\begin{array}{l}
1 \\
1
\end{array}\right]=\left[\begin{array}{l}
4 \\
6
\end{array}\right],
$$

where $I_{2}$ is the $2 \times 2$ identity matrix. If the initial state of the absorbing Markov chain is 2, it is well-known in the absorbing Markov chain literature (see, e.g., Kemeny and Snell 1960) that the chain will be absorbed by state $h$ within $T_{2}=6$ expected periods of event time. Therefore, if player $i$ deviates by increasing her threshold perpetually to $\bar{k}_{H}^{\prime}=2$, the expected continuation payoff is $U_{H}(h)-\frac{6 c}{2 p(1-p)}$. Such deviation is not strictly profitable if

$$
U_{H}(l) \geq U_{H}(h)-\frac{6 c}{2 p(1-p)},
$$

which is equivalent to

$$
\frac{p(1-p)\left(U_{H}(h)-U_{H}(l)\right)}{c} \leq 3=\frac{\left(\bar{k}_{H}+1\right)\left(\bar{k}_{H}+2\right)}{2} .
$$

A generalization of (3) and (4) under an arbitrary threshold $\bar{k}_{H} \in \mathbb{Z}_{+}$yields the following.

LEMMA 3 (Thresholds under LIFO). In all stationary* equilibria under LIFO in which $\mathrm{H}$ squares and $h$-rounds use threshold strategies, in all periods, $-\bar{k}^{\text {lifo }} \leq k_{H h} \leq \bar{k}^{\text {lifo }}$, where ${ }^{32}$

$$
\bar{k}^{\text {lifo }} \equiv\left\lfloor\sqrt{\frac{2 p(1-p)\left(U_{H}(h)-U_{H}(l)\right)}{c}+\frac{1}{4}}-\frac{1}{2}\right\rfloor .
$$

We now turn to the decisions of $l$-rounds (or, analogously, those of $L$-squares). An $l$-round matches with an $H$-square only when that $H$-square arrives with an $l$-round. LIFO then prescribes the $H$-square to be matched with the last $l$-round to enter the market. It follows that, if an $l$-round remains unmatched in the period of his arrival, he will

\footnotetext{
${ }^{31}$ Take any event time $\tau$, and suppose that the state at time $\tau$ is 2: i.e., there is another $H$-square waiting, who arrived after player $i$. The event time $\tau$ progresses to $\tau+1$ by an arrival of an incongruent pair. If the incongruent pair is $(L, h)$, the rank of player $i$ moves up to 1 . This transition occurs with probability $Q_{21}=1 / 2$. Otherwise, the new incongruent pair is $(H, l)$. According to $\psi_{H}$, the $H$-square who has been waiting with player $i$ demands an $l$-round and leaves the market, leaving player $i$ 's rank at 2 . This transition occurs with probability $Q_{22}=1 / 2$. If the state in period $\tau$ is 1 , and $(L, h)$ arrives, then player $i$ matches to the $h$-round. This last transition occurs with probability $R_{11}=1 / 2$.

${ }^{32}$ In the Appendix, we show that in all stationary* equilibria, the full support of the $H$ - $h$ queue is $\left\{-\bar{k}^{\text {lifo }}, . ., \bar{k}^{\text {lifo }}\right\}$. Therefore, the bounds described in Lemma 3 are achieved in equilibrium.
} 
never be matched with an $H$-square later. Therefore, $l$-rounds are incentivized to depart as soon as possible:

Lemma 4 (Equilibrium under LIFO). There exists a stationary* equilibrium in which $\mathrm{H}$ squares and $h$-rounds use a threshold $\bar{k}$ lifo and in which there can never be both $L$-squares and l-rounds waiting in the market. This equilibrium is welfare-maximizing among all stationary* equilibria.

Similar to Section 4, we can characterize the equilibrium steady state.

Proposition 5 (Discretionary steady state under LIFO). The welfare-maximizing stationary* equilibrium under LIFO is associated with a unique steady-state distribution over queue lengths, such that the length of the $H$-h queue $k_{H h}=k_{H}-k_{h}$ is uniformly distributed over $\left\{-\bar{k}^{\text {lifo }}, \ldots, \bar{k}^{\text {lifo }}\right\}$ and, in any period, the queues contain only $H$-squares and l-rounds or only $h$-rounds and $L$-squares.

We can now compare this threshold, as well as consequent welfare levels, to those emerging from the other protocols discussed throughout the paper.

COROLLARY 5 (Thresholds and welfare comparisons under LIFO).

(i) For sufficiently small waiting costs $c$, the maximal waiting queues under LIFO are longer than under the optimal mechanism, but shorter than under FIFO: $\bar{k}^{\text {opt }}<$ $\bar{k}^{\text {lifo }}<\bar{k}^{\text {fifo }}$.

(ii) The LIFO protocol is asymptotically efficient, i.e., the maximum equilibrium welfare under LIFO is given by $W^{\text {lifo }}(c)=S_{\infty}-\Gamma(c)$, where $\lim _{c \rightarrow 0} \Gamma(c)=0$.

Corollary 5 suggests that the LIFO protocol could represent a substantial improvement with respect to the FIFO protocol in discretionary settings, at least for small costs. Figure 3 depicts the welfare losses generated by both the FIFO and the LIFO protocols with respect to the optimal mechanism for the parameter values used in Figure 2: $U_{H}(h)=U_{h}(H)=3, U_{H}(l)=U_{h}(L)=U_{L}(h)=U_{l}(H)=1, U_{L}(l)=U_{l}(L)=0$, and $p=0.3$. The figure illustrates that the welfare gap decreases significantly under LIFO, even for costs far away from zero.

\section{Asymmetric markets}

Throughout, we assumed a symmetric environment in terms of waiting costs and type distributions. In this section, we consider a market with asymmetric type distributions, utilities, and waiting costs. Specifically, we assume the probability that a square is an $H$-square is $p_{H}$, while the probability that a round is an $h$-round is $p_{h}$ such that, without loss of generality, $p_{H} \geq p_{h}$. Furthermore, we allow for waiting costs to differ across market sides: we denote by $c_{S}$ and $c_{R}$ the per-period cost experienced by squares and rounds, respectively. We place no restrictions on match utilities other than that they 


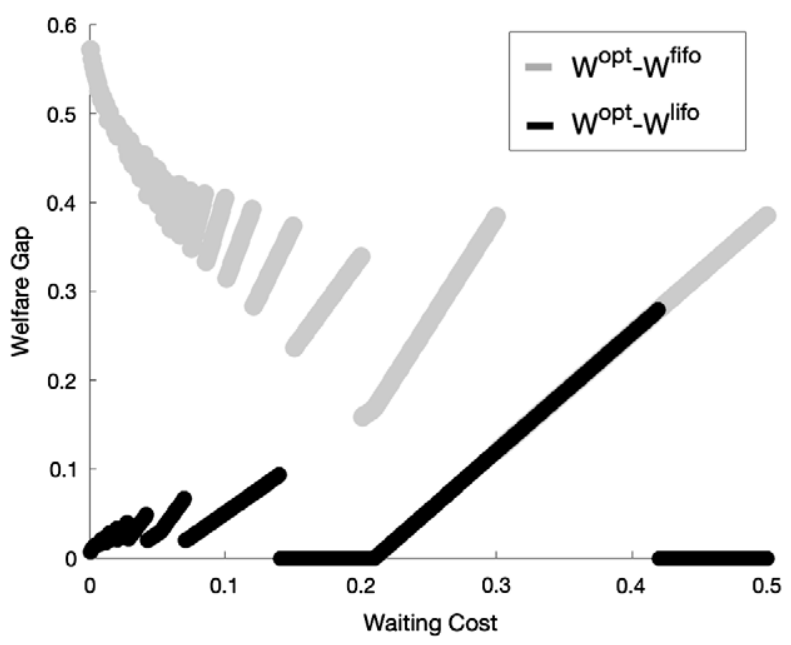

FIGURE 3. Welfare gaps between optimal and discretionary matching (FIFO and LIFO) as a function of costs.

are assortative and supermodular. In this environment, we summarize the characterization of the optimal mechanism and analyze a simpler one-threshold mechanism that approximates it with small waiting costs. Finally, we illustrate that the comparison between centralized and discretionary processes studied above carries through qualitatively in this more general environment.

\subsection{Optimal dynamic mechanism}

As seen in Section 3, when $p_{H}=p_{h}$, asymmetries in utilities play no role in the characterization of the optimal mechanism, whose welfare depends on joint match surpluses $U_{x y}=U_{x}(y)+U_{y}(x)$, for $x=H, L$, and $y=h$, l. Similarly, the optimal mechanism accounts for waiting costs incurred by pairs, $c_{S}+c_{R}$. An optimal mechanism can then be derived from an optimal mechanism when waiting costs for squares and rounds coincide and are equal to $c \equiv \frac{c_{S}+c_{R}}{2}$. Our focus here is, therefore, on the impact of asymmetries in type distributions on our results, the case in which $p_{H}>p_{h}$. As in the symmetric market, $(H, h)$ and $(L, l)$ pairs are matched immediately when available, and we focus on dynamic mechanisms that are identified by a pair of thresholds $\left(\bar{k}_{H}, \bar{k}_{h}\right)$. These thresholds do not necessarily coincide when type distributions differ for squares and rounds. Intuitively, since $H$-squares are more prevalent than $h$-rounds, it is more valuable for the mechanism designer to hold on to $(L, h)$ pairs in the hopes of $H$-squares appearing in the market than it is to hold on to $(H, l)$ pairs. As in Section 3, given a pair of thresholds $\left(\bar{k}_{H}, \bar{k}_{h}\right)$, we find the resulting net expected timeaverage welfare at the steady state. We look for the pair $\left(\bar{k}_{H}^{\text {opt }}, \bar{k}_{h}^{\text {opt }}\right)$ that maximizes this objective.

Recall that $s_{H h}^{t}$ denotes the value of the (signed) length of the $H$ - $h$ queue at the beginning of time $t$. $\mathbf{x}^{t} \in\{0,1\}^{\bar{k}_{H}+k_{h}+1}$ is the timed vector such that $x_{i}^{t}$ takes the value of 1 
if the state is $s_{H h}^{t}$ and 0 otherwise. Then

$$
\mathbf{x}^{t+1}=\mathbf{T}_{\bar{k}_{H}, \bar{k}_{h}} \mathbf{x}^{t}
$$

where

$$
\mathbf{T}_{\bar{k}_{H}, \bar{k}_{h}}=\left(\begin{array}{ccccc}
1-\left(1-p_{H}\right) p_{h} & p_{H}\left(1-p_{h}\right) & \ldots & 0 & 0 \\
\left(1-p_{H}\right) p_{h} & p_{H} p_{h}+\left(1-p_{H}\right)\left(1-p_{h}\right) & \cdots & 0 & 0 \\
0 & \left(1-p_{H}\right) p_{h} & \cdots & 0 & 0 \\
\vdots & \vdots & \ddots & \vdots & \vdots \\
0 & 0 & \cdots & p_{H}\left(1-p_{h}\right) & 0 \\
0 & 0 & \cdots & p_{H} p_{h}+\left(1-p_{H}\right)\left(1-p_{h}\right) & p_{H}\left(1-p_{h}\right) \\
0 & 0 & \cdots & \left(1-p_{H}\right) p_{h} & 1-p_{H}\left(1-p_{h}\right)
\end{array}\right) .
$$

Since the above Markov chain is ergodic, the corresponding matching process reaches a unique steady state with a distribution $\pi \equiv\left(\pi_{\bar{k}_{H}}, \pi_{\bar{k}_{H}-1}, \ldots, \pi_{-\bar{k}_{h}}\right)$ that we now identify. Denote

$$
\eta \equiv p_{H}\left(1-p_{h}\right)+\left(1-p_{H}\right) p_{h} \quad \text { and } \quad \phi \equiv \frac{\left(1-p_{H}\right) p_{h}}{p_{H}\left(1-p_{h}\right)} \quad(<1) .
$$

Rewriting $\mathbf{T}_{\bar{k}_{H}, \bar{k}_{h}}$ in terms of $\eta$ and $\phi$, we obtain

$$
\begin{aligned}
\pi_{\bar{k}_{H}} & =\left(1-\eta+\frac{\eta}{\phi+1}\right) \pi_{\bar{k}_{H}}+\frac{\eta}{\phi+1} \pi_{\bar{k}_{H}-1} \Longrightarrow \pi_{\bar{k}_{H}-1}=\phi \pi_{\bar{k}_{H}}, \\
\pi_{\bar{k}_{H}-1} & =\frac{\eta \phi}{\phi+1} \pi_{\bar{k}_{H}}+(1-\eta) \pi_{\bar{k}_{H}-1}+\frac{\eta}{\phi+1} \pi_{\bar{k}_{H}-2} \Longrightarrow \pi_{\bar{k}_{H}-2}=\phi \pi_{\bar{k}_{H}-1}=\phi^{2} \pi_{\bar{k}_{H}}, \\
& \vdots \\
\pi_{-\bar{k}_{H_{h}}} & =\frac{\eta \phi}{\phi+1} \pi_{-\bar{k}_{h}+1}+\left(1-\eta+\frac{\eta \phi}{\phi+1}\right) \pi_{\bar{k}_{H}}+\frac{\eta}{\phi+1} \pi_{\bar{k}_{H}-1} \Longrightarrow \pi_{-\bar{k}_{h}}=\phi^{\bar{k}_{H}+\bar{k}_{h}} \pi_{\bar{k}_{H}} .
\end{aligned}
$$

Since $\sum_{k=0}^{\bar{k}_{H}+\bar{k}_{h}} \phi^{k} \pi_{\bar{k}_{H}}=1$, it follows that $\pi_{\bar{k}_{H}}=\frac{1-\phi}{1-\phi^{\bar{k}_{H}+\bar{k}_{h}+1}}$. Therefore,

$$
\pi_{\bar{k}_{H}-k}=\frac{(1-\phi) \phi^{k}}{1-\phi^{\bar{k}_{H}+\bar{k}_{h}+1}} \quad \text { for every } k=0,1, \ldots, \bar{k}_{H}+\bar{k}_{h} .
$$

The expected time-average match surplus at the steady state is then

$$
\begin{aligned}
S\left(\bar{k}_{H}, \bar{k}_{h}\right)= & p_{H} p_{h} U_{H h}+\left(1-p_{H}\right)\left(1-p_{h}\right) U_{L l} \\
& +\mathbf{1}\left\{k_{H}>0\right\}\left(\sum_{k=1}^{\bar{k}_{H}} \phi^{\bar{k}_{H}-k} \pi_{\bar{k}_{H}}\left(1-p_{H}\right) p_{h}\left(U_{H h}+U_{L l}\right)\right) \\
& +\mathbf{1}\left\{k_{h}>0\right\}\left(\sum_{k=1}^{\bar{k}_{h}} \phi^{\bar{k}_{H}+k} \pi_{\bar{k}_{H}} p_{H}\left(1-p_{h}\right)\left(U_{H h}+U_{L l}\right)\right) \\
& +\pi_{\bar{k}_{H}} p_{H}\left(1-p_{h}\right) U_{H l}+\phi^{\bar{k}_{H}+\bar{k}_{h}} \pi_{\bar{k}_{H}}\left(1-p_{H}\right) p_{h} U_{L h} .
\end{aligned}
$$


The expected time-average waiting costs at the steady state are

$$
C\left(\bar{k}_{H}, \bar{k}_{h}\right)=2 c \pi_{\bar{k}_{H}}\left(\sum_{k=0}^{\bar{k}_{H}} k \phi^{\bar{k}_{H}-k}+\sum_{k=0}^{\bar{k}_{h}} k \phi^{\bar{k}_{H}+k}\right) .
$$

The optimal dynamic mechanism is identified by the pair of thresholds $\left(\bar{k}_{H}^{\text {opt }}, \bar{k}_{h}^{\text {opt }}\right)$ that maximizes the expected time-average welfare, $S\left(\bar{k}_{H}, \bar{k}_{h}\right)-C\left(\bar{k}_{H}, \bar{k}_{h}\right)$.

\subsection{One-threshold mechanisms}

When $H$-squares are strictly more likely to arrive than $h$-rounds $\left(p_{H}>p_{h}\right)$, there is a relatively small chance that many $h$-rounds arrive at the market and are not matched with $H$-squares. In other words, the (signed) length of the $H$ - $h$ queue is unlikely to reach very negative values. Therefore, we can consider a simpler mechanism, which only limits the length of the queue of $H$-squares. It turns out that the most efficient one-threshold mechanism, despite being less efficient than the optimal mechanism, is asymptotically efficient as waiting costs, $c_{S}$ and $c_{R}$, vanish. We find the expected total welfare for one period of time of the two-threshold dynamic mechanism $\left(\bar{k}_{H}, \bar{k}_{h}\right)$ as $\bar{k}_{h}$ becomes infinitely large. ${ }^{33}$ In the limit, $\pi_{\bar{k}_{H}-k}=(1-\phi) \phi^{k}$ for every $k=0,1,2, \ldots$ By applying this limit steady-state distribution, we obtain the corresponding limit match surplus, $S\left(\bar{k}_{H}, \infty\right)$, and waiting costs, $C\left(\bar{k}_{H}, \infty\right)$ :

$$
\begin{aligned}
S\left(\bar{k}_{H}, \infty\right)= & p_{H} p_{h} U_{H h}+\left(1-p_{H}\right)\left(1-p_{h}\right) U_{L l} \\
& +\mathbf{1}\left\{k_{H}>0\right\}\left(\sum_{k=0}^{\bar{k}_{H}-1}(1-\phi) \phi^{k}\left(1-p_{H}\right) p_{h}\left(U_{H h}+U_{L l}\right)\right) \\
& +\left(\sum_{k=1}^{\infty}(1-\phi) \phi^{\bar{k}_{H}+k} p_{H}\left(1-p_{h}\right)\left(U_{H h}+U_{L l}\right)\right) \\
& +(1-\phi) p_{H}\left(1-p_{h}\right) U_{H l}
\end{aligned}
$$

and

$$
C\left(\bar{k}_{H}, \infty\right)=2 c(1-\phi)\left(\sum_{k=0}^{\bar{k}_{H}} k \phi^{\bar{k}_{H}-k}+\sum_{k=0}^{\infty} k \phi^{\bar{k}_{H}+k}\right)
$$

\footnotetext{
${ }^{33}$ Technically, a one-threshold mechanism defines a Markov chain with a countable state space $\left\{\ldots,-1,0,1, \ldots, \bar{k}_{H}\right\}$. However, when transitions toward state $\bar{k}_{H}$ occur with probability strictly higher than that of transitions away from state $\bar{k}_{H}$ (i.e., $p_{H}\left(1-p_{h}\right)>p_{h}\left(1-p_{H}\right)$ ), the steady-state probabilities for the truncated Markov chain defined by a two-threshold mechanism $\left(\bar{k}_{H}, \bar{k}_{h}\right)$ approach the steady-state probabilities for the untruncated Markov chain as $\bar{k}_{h}$ increases.
} 
We can simplify the above expressions to achieve, for every $\bar{k}_{H}=0,1,2, \ldots$,

$$
\begin{aligned}
& S\left(\bar{k}_{H}, \infty\right)=p_{h} U_{H h}+\left(1-p_{H}\right) U_{L l}+\left(p_{H}-p_{h}\right) U_{H l}=S_{\infty}, \quad \text { and } \\
& C\left(\bar{k}_{H}, \infty\right)=2 c(1-\phi) \phi^{\bar{k}_{H}}\left(\frac{\phi}{(1-\phi)^{2}}+\sum_{k=0}^{\bar{k}_{H}} k \phi^{-k}\right) .
\end{aligned}
$$

The expected time-average welfare is $W\left(\bar{k}_{H}\right) \equiv S\left(\bar{k}_{H}, \infty\right)-C\left(\bar{k}_{H}, \infty\right)$. We inspect the marginal time-average welfare with respect to the length of the queue of $H$-squares $\Delta_{+} W\left(\bar{k}_{H}\right) \equiv W\left(\bar{k}_{H}+1\right)-W\left(\bar{k}_{H}\right)$ and find the most efficient one-threshold $\bar{k}_{H}^{* *}$ from

$$
\Delta_{+} W\left(\bar{k}_{H}^{* *}\right) \leq 0 \leq \Delta_{+} W\left(\bar{k}_{H}^{* *}-1\right) .
$$

Now, to derive a closed-form solution for $\bar{k}_{H}^{* *}$, notice that the expected total surplus $S\left(\bar{k}_{H}, \infty\right)$ is a constant function of $\bar{k}_{H}$. Therefore,

$$
\begin{aligned}
\Delta_{+} W\left(\bar{k}_{H}\right)= & C\left(\bar{k}_{H}, \infty\right)-C\left(\bar{k}_{H}+1, \infty\right) \\
= & 2 c(1-\phi)\left(\phi^{\bar{k}_{H}}-\phi^{\bar{k}_{H}+1}\right)\left(\frac{\phi}{(1-\phi)^{2}}+\sum_{k=0}^{\bar{k}_{H}} k \phi^{-k}\right) \\
& +2 c(1-\phi) \phi^{\bar{k}_{H}+1}\left(\sum_{k=0}^{\bar{k}_{H}} k \phi^{-k}-\sum_{k=0}^{\bar{k}_{H}+1} k \phi^{-k}\right) \\
= & 2 c\left(2 \phi^{\bar{k}_{H}+1}-1\right) .
\end{aligned}
$$

The most efficient one-threshold mechanism is identified from (5) as

$$
\bar{k}_{H}^{* *}=\left\lfloor-\frac{\log 2}{\log \phi}\right\rfloor=\left\lfloor-\frac{\log 2}{\log \left(1-p_{H}\right)+\log p_{h}-\log p_{H}-\log \left(1-p_{h}\right)}\right\rfloor .
$$

This efficient threshold $\bar{k}_{H}^{* *}$ does not depend on $c$. Importantly, every fixed onethreshold mechanism is asymptotically efficient with vanishingly small waiting costs. Intuitively, in the one-threshold mechanism, an incongruent pair leaves the market only when the state is $k_{H h}=\bar{k}_{H}$, which always occurs with probability $1-\phi$ at the steady state. Therefore, all one-threshold mechanisms result in the same expected fraction of incongruent pairs matched in the steady state. In fact, the expected total time-average match surplus is $S_{\infty}$ regardless of the threshold $\bar{k}_{H}$. For any fixed threshold $\bar{k}_{H}$, as waiting costs vanish, the expected total time-average waiting costs approach zero and efficiency is achieved.

\subsection{Discretionary matching}

We focus on regular environments in which $p_{h}\left(U_{H}(h)-U_{H}(l)\right) \neq k c_{S}$ and $p_{H}\left(U_{h}(H)-\right.$ $\left.U_{h}(L)\right) \neq k c_{R}$ for every $k \in \mathbb{Z}_{+}$. The decisions of an $H$-square (analogously, an $h$-round) remain as described in Section 4. Namely, when an $H$-square arrives at the market and 
an $h$-round is available, an $(H, h)$ pair is formed immediately. If an $h$-round is not available, the arriving $H$-square decides to wait in the queue based on the number of $\mathrm{H}$ squares already in the queue. Since an $h$-round is not available, this implies that the $H$-square arrived with an $l$-round. As all $l$-rounds are willing to match with $H$-squares, the newly arrived $H$-square will wait if and only if the gain $U_{H}(h)-U_{H}(l)$ exceeds the expected waiting costs until matching with an $h$-round. In analogy with Lemma 1 , the (signed) length of the $H$ - $h$ queue at the beginning of a period, $k_{H h} \equiv k_{H}-k_{h}$, will then be bounded as $-\bar{k}_{h}^{\text {fifo }} \leq k_{H h} \leq \bar{k}_{H}^{\text {fifo }}$, where

$$
\begin{aligned}
& \bar{k}_{H}^{\text {fifo }} \equiv \max \left\{k \in \mathbb{Z}_{+} \mid \frac{k c_{S}}{p_{h}}<U_{H}(h)-U_{H}(l)\right\}, \quad \text { and } \\
& \bar{k}_{h}^{\text {fifo }} \equiv \max \left\{k \in \mathbb{Z}_{+} \mid \frac{k c_{R}}{p_{H}}<U_{h}(H)-U_{h}(L)\right\} .
\end{aligned}
$$

An $l$-round (similarly, an $L$-square) may decide to wait to match with an $H$-square if the queue of $H$-squares is long and expected to hit the threshold $\bar{k}_{H}^{\text {fifo }}$ within a sufficiently short time. In contrast with the symmetric case, $L$-squares and $l$-rounds may now wait simultaneously in equilibrium. Intuitively, consider an environment in which both types of rounds are nearly indifferent between matching with $H$-squares or $L$ squares and, therefore, match with whomever is available immediately. In such an environment, an $L$-square, who is first in line, may decide to wait, even when arriving with an $l$-round, in the hopes of an $(L, h)$ pair arriving in the next period. In other words, in general asymmetric markets, Lemma 2 does not hold. A full characterization of the equilibrium requires the analysis of a rather complex random process of the 3-dimensional vector $\left(k_{H h}, k_{L}, k_{l}\right)$. In order to achieve bounds on equilibrium welfare, we study a one-dimensional Markov process of $k_{H h}$ only, with a transition matrix as described in Section 7.1. In equilibrium, as well as under the onedimensional protocol discussed above, the expected time-average surplus is bounded above by $S_{\infty}=p_{h} U_{H h}+\left(1-p_{H}\right) U_{L l}+\left(p_{H}-p_{h}\right) U_{H l}$. In the one-dimensional process with thresholds $\bar{k}_{h}^{\text {fifo }}$ and $\bar{k}_{H}^{\text {fifo }}$, at each state $k_{H h}$, either $k_{H h} H$-squares (and at least as many $l$-rounds) or $\left|k_{H h}\right| h$-rounds (and at least as many $L$-squares) incur waiting costs. Since in equilibrium there might be additional waiting costs incurred through the simultaneous waiting of $L$-squares or $l$-rounds, the resulting per-period welfare $W^{\text {fifo }}\left(c_{S}, c_{R}\right)$ can be bounded as follows:

$$
W^{\text {fifo }}\left(c_{S}, c_{R}\right) \leq S_{\infty}-\left(c_{S}+c_{R}\right) \pi_{\bar{k}_{H}^{\text {fifo }}}\left(\sum_{k=0}^{\bar{k}_{H}^{\text {fifo }} k} \phi^{\bar{k}_{H}^{\text {fifo }}-k}+\sum_{k=0}^{\bar{k}_{h}^{\text {fifo }}} k \phi^{\bar{k}_{H}^{\text {fifo }}+k}\right) .
$$

After some algebraic manipulation, we can show that

$$
\lim _{\left(c_{S}, c_{R}\right) \rightarrow(0,0)} W^{\text {fifo }}\left(c_{S}, c_{R}\right) \leq S_{\infty}-p_{h}\left(U_{H}(h)-U_{H}(l)\right) .
$$

This echoes Corollary 4 . The bound on the welfare wedge between the discretionary protocol and the optimal mechanism exhibits similar comparative statics to those described for symmetric environments, increasing in $p_{h}$ and in $U_{H}(h)-U_{H}(l)$. 


\section{APPENDIX}

\section{A.1 Proofs regarding the optimal mechanism}

We start with a preliminary lemma. ${ }^{34}$

Lemma A1. (i) For any mechanism $\mu$, there exists a mechanism $\mu^{\prime}$, with $v\left(\mu^{\prime}\right) \geq v(\mu)$, which never holds $H$-squares and $h$-rounds that are both available, or $L$-squares and $l$ rounds that are both available; (ii) For any mechanism $\mu$, there exists a mechanism $\mu^{\prime}$, with $v\left(\mu^{\prime}\right) \geq v(\mu)$, which never holds more than $\frac{U}{2 c}$ squares (and rounds) in the market.

Due to Lemma A1, the planner essentially solves the following Markov decision problem with agents arriving in incongruent pairs, a finite set of states, and a finite set of actions:

$$
\left(\mathrm{MDP}, s^{0}\right) \equiv\left\{T, S, s^{0},(r(s, k), p(\cdot \mid k))_{s \in S, k \in H_{s}}\right\},
$$

where $s^{0}$ denotes a particular initial state. Each component is defined as follows:

1. $T \equiv\{0,1,2, \ldots\}$ is the set of event times. As described in the body of the paper, event times correspond to times at which incongruent pairs $(H, l)$ or $(L, h)$ arrive. Since the probability of an incongruent pair arriving at any period is $2 p(1-p)$, the expected time between event times is $\frac{1}{2 p(1-p)}$.

2. $S \equiv\{z \in \mathbb{Z}:-(U / 2 c)-1 \leq z \leq(U / 2 c)+1\}$ is the set of possible states (or stocks). Each state $s_{H h} \equiv s_{H}-s_{h} \in S$ represents the (signed) number of incongruent pairs of type $(H, l)$ or $(L, h)$ in the market. Since we restrict our attention to mechanisms that do not hold more than $U / 2 c$ squares (and rounds), a state, which takes a new arriving pair into account, has to belong to the set $\{-\lfloor U / 2 c\rfloor-1, \ldots,\lfloor U / 2 c\rfloor+1\}$.

3. $s^{0}=0$ is the the initial state. Initially, there is no agent waiting in the market.

4. $K \equiv\{z \in \mathbb{Z}:-U / 2 c \leq z \leq U / 2 c\}$ is the set of available actions. Each $k \in K$ represents the (signed) number of incongruent pairs held in the market from one period to the next.

5. $r(s, k)$ is the reward function: for every $s \in S, k \in K$,

$$
r(s, k)= \begin{cases}(s-k) U_{H l}-\frac{k c}{2 p(1-p)} & \text { if } s \geq k \geq 0 \\ (|s|-|k|) U_{L h}-\frac{|k| c}{2 p(1-p)} & \text { if } s \leq k \leq 0 \\ -\infty & \text { otherwise }\end{cases}
$$

The expected waiting cost incurred by any agent who waits for one event time is $\frac{c}{2 p(1-p)}$. The reward function returns $-\infty$ if an action is infeasible. For all feasible actions, the values of the reward function are in the interval $\left[-\frac{U}{4 p(1-p)},\left(\frac{U}{2 c}+\right.\right.$ 1) $\left.U_{H h}\right]$.

\footnotetext{
${ }^{34} \mathrm{~A}$ formal proof of Lemma Al follows standard arguments and is available from the authors upon request.
} 
6. $p(s, k)$ is the transition probability, the probability the system is in state $s \in S$ at any time $\tau+1$, after the action $k$ has been chosen at time $\tau$.

$$
p(s, k)= \begin{cases}1 / 2 & \text { for } s=k-1, k+1, \\ 0 & \text { otherwise }\end{cases}
$$

(MDP, $s^{0}$ ) is stationary in the sense that the reward function $r(s, k)$ and the transition probability function $p(s, k)$ do not depend on time, or event times, explicitly. A policy of $\left(\mathrm{MDP}, s^{0}\right)$ is any rule, deterministic or randomized, governing the choice of actions. Such a rule may, in principle, be history-dependent. The value of a policy $\mu$ is then

$$
v(\mu) \equiv \liminf _{T \rightarrow \infty} \frac{1}{T} E_{\mu}\left[\sum_{\tau=1}^{T} r\left(s^{\tau}, k^{\tau}\right)\right] .
$$

A stationary and deterministic policy, which we call an SD-policy, of (MDP, $s^{0}$ ) applies the same deterministic decision rule $\mu^{\mathrm{SD}}: S \rightarrow K$ regardless of the history. The value of $\mu^{\mathrm{SD}}$ is then

$$
v\left(\mu^{\mathrm{SD}}\right)=\lim _{T \rightarrow \infty} \frac{1}{T} E\left[\sum_{\tau=1}^{T} r\left(s^{\tau}, \mu^{\mathrm{SD}}\left(s^{\tau}\right)\right)\right] .
$$

The limit exists, as guaranteed, for example, by Proposition 8.1.1(b) in Puterman (2005). The finite state space implies the existence of an optimal SD-policy (see Theorem 7.1.9 of Puterman (2005) or Theorem Puterman 3 below). As such, we can focus on SDmechanisms satisfying Assumptions 1 and 2 in Section 3 without loss of generality.

Proof of Proposition 1. The proof follows several steps.

Step 1 (Existence of Thresholds $\left(\bar{k}_{H}, \bar{k}_{h}\right)$ )

Any stationary and deterministic policy $d$ of (MDP) is associated with two thresholds, representing the largest number of $(H, l)$ and $(L, h)$ pairs held in the market at any time, respectively. ${ }^{35}$ Define

$$
\begin{aligned}
\bar{k}_{H} & \equiv \min \{s \mid s>0, d(s)<s\}-1, \quad \text { and } \\
\bar{k}_{h} & \equiv \min \{|s| \mid s<0, d(s)>s\}+1 .
\end{aligned}
$$

The thresholds $\left(\bar{k}_{H}, \bar{k}_{h}\right)$ are well-defined. Indeed, policies maintain only a bounded number of unmatched pairs in the market. We claim that the value of a policy is uniquely determined by the thresholds and decisions at the thresholds. Given policy $d$, define

$$
d^{\prime}(s) \equiv \begin{cases}d(s) & \text { if }-\bar{k}_{h} \leq s \leq \bar{k}_{H} \\ d\left(\bar{k}_{H}\right) & \text { if } s>\bar{k}_{H} \\ d\left(-\bar{k}_{h}\right) & \text { if } s<-\bar{k}_{h} .\end{cases}
$$

\footnotetext{
${ }^{35}$ Indeed, suppose a policy dictates matches to be formed when the number of, say, $(H, l)$ pairs exceeds $k_{H}^{1}$ or $k_{H}^{2}>k_{H}^{1}$. The number of $(H, l)$ pairs would then never surpass $k_{H}^{2}$, so the relevant threshold for outcomes would be the minimal threshold $k_{H}^{1}$.
} 
The Markov processes induced by $d$ and $d^{\prime}$, namely $\left\{\left(s^{\tau}, r\left(s^{\tau}, d\left(s^{\tau}\right)\right)\right\}_{\tau=0}^{\infty}\right.$ and $\left\{\left(s^{\tau}, r\left(s^{\tau}, d^{\prime}\left(s^{\tau}\right)\right)\right)\right\}_{\tau=0}^{\infty}$, are identical. Thus, $v(d)=v\left(d^{\prime}\right)$. We can therefore characterize any policy $d$ by its corresponding thresholds $\left(\bar{k}_{H}, \bar{k}_{h}\right)$ and decisions at the thresholds $\left(d\left(\bar{k}_{H}\right), d\left(\bar{k}_{h}\right)\right) .{ }^{36}$

\section{Step 2 (Stationary distribution of $k_{H h}$ )}

We characterize the unique stationary distribution of $k_{H h}$ corresponding to the ergodic Markov process induced by a policy $d$.

Claim 1. Take $\bar{k}_{H}, \bar{k}_{h} \in\left[1, \frac{U}{2 c}\right] \cap \mathbb{Z}_{+}$, and $z_{H}, z_{h} \in \mathbb{Z}_{+}$with $z_{H} \leq \bar{k}_{H}$ and $z_{h} \leq \bar{k}_{h}$. A policy $d$ of (MDP) defined by

$$
d(s) \equiv \begin{cases}s & \text { if }-\bar{k}_{h} \leq s \leq \bar{k}_{H} \\ \bar{k}_{H}-z_{H} & \text { if } s>\bar{k}_{H} \\ -\bar{k}_{h}+z_{h} & \text { if } s<-\bar{k}_{h} .\end{cases}
$$

induces a Markov chain corresponding to $k_{H h}$. The unique steady-state distribution $\boldsymbol{\pi}=$ $\left(\pi_{-\bar{k}_{h}}, \ldots, \pi_{\bar{k}_{H}}\right)$ is such that:

(i) (Middle range) for $-\bar{k}_{h}+z_{h} \leq k \leq \bar{k}_{H}-z_{H}, \pi_{k}=\pi_{0}=\frac{1}{\bar{k}_{H}+\bar{k}_{h}-z_{H} / 2-z_{h} / 2+1}$,

(ii) (Upper range) for $z=1, \ldots, z_{H}, \pi_{\bar{k}_{H}-z_{H}+z}=\pi_{0}\left(1-\frac{z}{z_{H}+1}\right)$,

(iii) (Lower range) for $z=1, \ldots, z_{h}, \pi_{-\bar{k}_{h}+z_{h}-z}=\pi_{0}\left(1-\frac{z}{z_{h}+1}\right)$.

That is, the stationary distribution is uniform in the middle range. The stationary probability mass decreases as $k_{H h}$ approaches $\bar{k}_{H}$ or $\bar{k}_{h}$.

Proof of Claim 1. Denote by

$$
\mathbf{x}^{\tau} \equiv\left(x_{-\bar{k}_{h}}^{\tau}, x_{-\bar{k}_{h}+1}^{\tau}, \ldots, x_{\bar{k}_{H}-1}^{\tau}, x_{\bar{k}_{H}}^{\tau}\right)^{t r} \in\{0,1\}^{\bar{k}_{H}+\bar{k}_{h}+1}
$$

the timed vector such that $x_{i}^{\tau}=\mathbf{1}\left(k_{H h}=i\right)$. Then, $\mathbf{x}^{\tau+1}=\mathbf{T}_{d} \mathbf{x}^{\tau}$, where

$$
\mathbf{T}_{d}=\left(\begin{array}{ccccc}
0 & 1 / 2 & \ldots & 0 & 0 \\
1 / 2 & 0 & \ldots & 0 & 0 \\
0 & 1 / 2 & \ldots & 0 & 0 \\
\vdots & \vdots & \ddots & \vdots & \vdots \\
0 & 0 & \ldots & 1 / 2 & 0 \\
0 & 0 & \ldots & 0 & 1 / 2 \\
0 & 0 & \ldots & 1 / 2 & 0
\end{array}\right)+\left(\begin{array}{ccccc}
0 & 0 & \ldots & 0 & 0 \\
\vdots & \vdots & \ldots & \vdots & \vdots \\
1 / 2 & 0 & \ldots & 0 & 0 \\
\vdots & \vdots & \ddots & \vdots & \vdots \\
0 & 0 & \ldots & 0 & 1 / 2 \\
\vdots & \vdots & \ldots & \vdots & \vdots \\
0 & 0 & \ldots & 0 & 0
\end{array}\right) .
$$

\footnotetext{
${ }^{36}$ There is multiplicity regarding prescriptions for states that are never reached. With thresholds $\bar{k}_{H}$ and $\bar{k}_{h}$ the market never has more than $\bar{k}_{H}+1 H$-squares or more than $\bar{k}_{h}+1 h$-rounds. The specification of what happens outside of these regions therefore has no impact on outcomes.
} 
The second matrix on the right-hand side has two non-zero elements valued at $1 / 2$. Each represents two scenarios, a transition from $\bar{k}_{H}$ upon the arrival of an $(H, l)$ pair to $\bar{k}_{H}-z_{H}$, and a transition from $-\bar{k}_{h}$ upon the arrival of a $(L, h)$ pair to $-\bar{k}_{h}+z_{h}$. The first matrix includes all other transitions. The Markov chain is ergodic, and the unique stationary distribution of $k_{H h}$ exists. Then $\pi$ in Claim 1 is the unique stationary distribution using straightforward calculations.

\section{Step 3 (Welfare)}

We compute the average welfare (i.e., total welfare per period) for any stationary and deterministic mechanism $\mu$. Let $d$ be the associated policy of (MDP) with thresholds $\left(\bar{k}_{H}, \bar{k}_{h}\right)$ and the decisions at the thresholds identified by $\left(z_{H}, z_{h}\right) \cdot{ }^{37}$

First, we compute the average total surplus generated in one time period. A newly arrived pair is of type $(H, h)$ with probability $p^{2}$, in which case the optimal mechanism generates a surplus equal to $U_{H h}$. Similarly, a newly arrived pair is of type $(L, l)$ with probability $(1-p)^{2}$, in which case the optimal mechanism generates a surplus equal to $U_{L l}$.

Suppose an $(H, l)$ pair arrives at time $t$ when the stock is $k_{H h}^{t-1}$. If $k_{H h}^{t-1}<0$, the mechanism creates one $(H, h)$ and one $(L, l)$ pair, generating a surplus equal to $U_{H h}+U_{L l}$. If $0 \leq k_{H h}^{t-1}<\bar{k}_{H}$, the mechanism creates no matches (and no additional surplus), and if $k_{H h}^{t-1}=\bar{k}_{H}$, the mechanism creates $\left(z_{H}+1\right)$ matches of $(H, l)$ pairs. Analogous conclusions pertain to the case in which a $(L, h)$ pair arrives. The expected match surplus per period is therefore

$$
\begin{aligned}
p^{2} U_{H h}+(1-p)^{2} U_{L l}+\left(1-\pi_{0}\right) p(1-p)\left(U_{H h}+U_{L l}\right) \\
\quad+\pi_{\bar{k}_{H}} p(1-p)\left(z_{H}+1\right) U_{H l}+\pi_{\bar{k}_{h}} p(1-p)\left(z_{h}+1\right) U_{L h} \\
=p U_{H h}+(1-p) U_{L l}-\pi_{0} p(1-p) U .
\end{aligned}
$$

Next, we compute the average total waiting costs incurred by agents waiting in line for one period. During the transition from time $t$ culminating at stock $k_{H h}$ to time $t+$ $1,2\left|k_{H h}\right|$ agents wait in line so the total costs of waiting incurred during this one time period are $2\left|k_{H h}\right| c$. Thus, a mechanism with thresholds $\left(\bar{k}_{H}, \bar{k}_{h}\right)$ results in expected total costs of waiting equal to

$$
\sum_{k=1}^{\bar{k}_{H}} 2 c \pi_{k}|k|+\sum_{k=-1}^{-\bar{k}_{h}} 2 c \pi_{k}|k|
$$

The first term equals to

$$
\begin{gathered}
\left(2 c \pi_{0}\right) \sum_{k=1}^{\bar{k}_{H}-z_{H}} k+\left(2 c \pi_{0}\right) \sum_{z=1}^{z_{H}}\left(1-\frac{1}{z_{H}+1}\right)\left(\bar{k}_{H}-z_{H}+z\right) \\
=\left(c \pi_{0}\right)\left(\left(\bar{k}_{H}-z_{H}\right)\left(\bar{k}_{H}+1\right)+\frac{z_{H}\left(z_{H}+2\right)}{3}\right) .
\end{gathered}
$$

${ }^{37}$ That is, for $s>\bar{k}_{H}, d(s)=\bar{k}_{H}-z_{H}$ and for $s<-\bar{k}_{h}, d(s)=-\bar{k}_{h}+z_{h}$. 
The second term is computed similarly. The average welfare of the mechanism $\mu$ is then

$$
\begin{aligned}
& W\left(\bar{k}_{H}, \bar{k}_{h}, z_{H}, z_{h}\right) \\
& \quad=p U_{H h}+(1-p) U_{L l}-\pi_{0} p(1-p) U \\
& \quad-\left(c \pi_{0}\right)\left(\left(\bar{k}_{H}-z_{H}\right)\left(\bar{k}_{H}+1\right)+\left(\bar{k}_{h}-z_{h}\right)\left(\bar{k}_{h}+1\right)+\frac{z_{H}\left(z_{H}+2\right)+z_{h}\left(z_{h}+2\right)}{3}\right),
\end{aligned}
$$

where $\pi_{0}=\frac{2}{2 \bar{k}_{H}+2 \bar{k}_{h}-z_{H}-z_{h}+2}$.

Step 4 (Matching at most one pair at a time)

We now show that we can focus on mechanisms satisfying $z_{H}=z_{h}=0$. In fact, generically this restriction is necessary for a mechanism to be optimal. The proof follows from the following claim, which completes the proof of Proposition 1.

Claim 2. Fix any $\bar{k}_{h}$ and $z_{h}\left(\leq \bar{k}_{h}\right)$. For any $\bar{k}_{H} \geq 1$ and $0 \leq z_{H} \leq \bar{k}_{H}-1$,

$$
W\left(\bar{k}_{H}, \bar{k}_{h}, z_{H}+1, z_{h}\right) \geq W\left(\bar{k}_{H}, \bar{k}_{h}, z_{H}, z_{h}\right)
$$

implies

$$
W\left(\bar{k}_{H}-1, \bar{k}_{h}, z_{H}, z_{h}\right) \geq W\left(\bar{k}_{H}, \bar{k}_{h}, z_{H}+1, z_{h}\right) .
$$

That is, whenever a mechanism with a larger $z_{H}$ leads to a higher average welfare, we can find a mechanism with an even higher average welfare by decreasing the threshold $\bar{k}_{H}$, while adhering to a smaller $z_{H}$.

Proof of Claim 2. Let

$$
\begin{aligned}
& \phi=2 \bar{k}_{H}+2 \bar{k}_{h}-z_{H}-z_{h}+1 \text { and } \\
& \psi=\left(\bar{k}_{H}-z_{H}\right)\left(\bar{k}_{H}+1\right)+\left(\bar{k}_{h}-z_{h}\right)\left(\bar{k}_{h}+1\right)+\frac{z_{H}\left(z_{H}+2\right)+z_{h}\left(z_{h}+2\right)}{3} .
\end{aligned}
$$

The first inequality in Claim 2 holds if and only if

$$
\frac{p(1-p) U}{\phi}+\frac{c}{\phi}\left(\psi-\bar{k}_{H}+\frac{2 z_{H}}{3}\right) \leq \frac{p(1-p) U}{\phi+1}+\frac{c}{\phi+1} \psi,
$$

or equivalently

$$
p(1-p) U+c \psi-(\phi+1) c\left(\bar{k}_{H}-\frac{2 z_{H}}{3}\right) \leq 0 .
$$

The second inequality in Claim 2 holds if and only if

$$
\frac{p(1-p) U}{\phi-1}+\frac{c}{\phi-1}\left(\psi-2 \bar{k}_{H}+z_{H}\right) \leq \frac{p(1-p) U}{\phi}+\frac{c}{\phi}\left(\psi-\bar{k}_{H}+\frac{2 z_{H}}{3}\right),
$$


or equivalently

$$
p(1-p) U+c \psi-c\left(\bar{k}_{H}-\frac{2 z_{H}}{3}\right)-\phi c\left(\bar{k}_{H}-\frac{z_{H}}{3}\right) \leq 0 .
$$

Clearly, (6) implies (7).

Claim 2 completes the proof of Proposition 1. Furthermore, this claim illustrates that there is always an optimal mechanism identified by $z_{H}=z_{h}=0$. From the proof, notice that if $z_{H}>0$, inequality (6) implies that inequality (7) holds with a strict inequality. Therefore, in any optimal mechanism, $z_{H}, z_{h}<2$. In fact, multiplicity can emerge only when there is multiplicity in the thresholds $\bar{k}_{H}, \bar{k}_{h}$ fixing $z_{H}=z_{h}=0$. Indeed, suppose there is an optimal mechanism with $\bar{k}_{H}$ and $z_{H}=1$ and some $\bar{k}_{h}, z_{h}$. From the proof of Claim 2, it follows that

$$
W\left(\bar{k}_{H}, \bar{k}_{h}, 1, z_{h}\right)-W\left(\bar{k}_{H}, \bar{k}_{h}, 0, z_{h}\right)=W\left(\bar{k}_{H}-1, \bar{k}_{h}, 0, z_{h}\right)-W\left(\bar{k}_{H}, \bar{k}_{h}, 1, z_{h}\right) .
$$

The optimality of $\bar{k}_{H}$ and $z_{H}=1$ implies that, in the above equality, both sides equal to 0 (otherwise, the mechanism identified by $\bar{k}_{H}-1$ and $z_{H}=0$, with $\bar{k}_{h}, z_{h}$, would generate greater welfare). In particular, there are optimal mechanisms identified by both $\bar{k}_{H}-1$ and $z_{H}=0$ as well as $\bar{k}_{H}$ and $z_{H}=0$.

Proof of Proposition 2. We find an optimal threshold pair $\left(\bar{k}_{H}, \bar{k}_{h}\right)$, assuming that $z_{H}=z_{h}=0$. To prove Proposition 2, we write the average welfare as

$$
p U_{H h}+(1-p) U_{L l}-\frac{p(1-p) U}{\bar{k}_{H}+\bar{k}_{h}+1}-\frac{\left(\bar{k}_{H}\left(\bar{k}_{H}+1\right)+\bar{k}_{h}\left(\bar{k}_{h}+1\right)\right) c}{\bar{k}_{H}+\bar{k}_{h}+1} .
$$

We use the following change of variables:

$$
\phi \equiv \bar{k}_{H}+\bar{k}_{h}, \quad \text { and } \quad \psi \equiv \bar{k}_{H}-\bar{k}_{h},
$$

and rewrite the above expression for welfare as

$$
p U_{H h}+(1-p) U_{L l}-\frac{p(1-p) U}{\phi+1}-\frac{\left(\phi^{2}+2 \phi+\psi^{2}\right) c}{2(\phi+1)} .
$$

The welfare is maximized when $\psi=0$ (i.e., $\bar{k}_{H}=\bar{k}_{h}=\frac{\phi}{2}$ ) if $\phi$ is even, or $|\psi|=1$ if $\phi$ is odd. We take into account this necessary condition of an optimal threshold pair and rewrite the welfare as

$$
W(\phi)= \begin{cases}p U_{H h}+(1-p) U_{L l}-\frac{p(1-p) U}{\phi+1}-\frac{(\phi+1) c}{2} & \text { if } \phi \text { is odd } \\ p U_{H h}+(1-p) U_{L l}-\frac{p(1-p) U}{\phi+1}-\frac{(\phi+1) c}{2}+\frac{c}{2(\phi+1)} & \text { if } \phi \text { is even. }\end{cases}
$$

Define the marginal increase of welfare when increasing the threshold by one as $\Delta_{+} W(\phi) \equiv W(\phi+1)-W(\phi)$. If $\phi \in \mathbb{Z}_{+}$is odd,

$$
\Delta_{+} W(\phi)=\frac{p(1-p) U}{(\phi+1)(\phi+2)}-\frac{c}{2}\left(\frac{\phi+1}{\phi+2}\right)
$$


If $\phi \in \mathbb{Z}_{+}$is even,

$$
\Delta_{+} W(\phi)=\frac{p(1-p) U}{(\phi+1)(\phi+2)}-\frac{c}{2}\left(\frac{\phi+2}{\phi+1}\right) .
$$

For nontrivial (i.e., nonzero) optimal thresholds, it is necessary that $\Delta_{+} W(0)>0$, or equivalently $c<\frac{p(1-p) U}{2}$. Suppose $c$ is small enough that this is the case. A necessary condition for an optimal sum of thresholds $\phi^{*}(\geq 1)$ is $\Delta_{+} W\left(\phi^{*}\right) \leq 0 \leq \Delta_{+} W\left(\phi^{*}-1\right)$. Thus, a necessary condition for an odd $\phi^{*}$ is

$$
\frac{p(1-p) U}{\left(\phi^{*}+1\right)\left(\phi^{*}+2\right)}-\frac{c}{2}\left(\frac{\phi^{*}+1}{\phi^{*}+2}\right) \leq 0 \leq \frac{p(1-p) U}{\phi^{*}\left(\phi^{*}+1\right)}-\frac{c}{2}\left(\frac{\phi^{*}+1}{\phi^{*}}\right),
$$

which is equivalent to

$$
\phi^{*}=\sqrt{\frac{2 p(1-p) U}{c}}-1
$$

Similarly, a necessary condition for an even $\phi^{*}$ is

$$
\frac{p(1-p) U}{\left(\phi^{*}+1\right)\left(\phi^{*}+2\right)}-\frac{c}{2}\left(\frac{\phi^{*}+2}{\phi^{*}+1}\right) \leq 0 \leq \frac{p(1-p) U}{\phi^{*}\left(\phi^{*}+1\right)}-\frac{c}{2}\left(\frac{\phi^{*}}{\phi^{*}+1}\right),
$$

which is equivalent to

$$
\sqrt{\frac{2 p(1-p) U}{c}}-2 \leq \phi^{*} \leq \sqrt{\frac{2 p(1-p) U}{c}}
$$

Therefore, an optimal thresholds sum $\phi^{*}$ must be even unless $\sqrt{\frac{2 p(1-p) U}{c}}$ is an even integer. The generically unique optimal threshold is identified by

$$
\bar{k}_{H}^{\mathrm{opt}}=\bar{k}_{h}^{\mathrm{opt}}=\frac{\phi^{*}}{2}=\left\lfloor\sqrt{\frac{p(1-p) U}{2 c}}\right\rfloor .
$$

It is easy to verify that, when $\sqrt{\frac{p(1-p) U}{2 c}}$ is an integer, any combination of thresholds $\left(\bar{k}_{H}^{\text {opt }}, \bar{k}_{h}^{\text {opt }}\right)$ such that $\bar{k}_{H}^{\text {opt }}, \bar{k}_{h}^{\text {opt }} \in\left\{\sqrt{\frac{p(1-p) U}{2 c}}, \sqrt{\frac{p(1-p) U}{2 c}}-1\right\}$ identifies an optimal mechanism. Furthermore, multiplicity emerges only when $\sqrt{\frac{p(1-p) U}{2 c}}$ is an integer.

Proof of Corollary 1. Using the optimal thresholds from Proposition 2, we get that for $c \leq \frac{p(1-p) U}{2}$,

$$
\begin{aligned}
& f(c) \equiv \frac{p(1-p) U}{2 \bar{k}^{\mathrm{opt}}+1}=\frac{p(1-p) U}{2\left\lfloor\sqrt{\left.\frac{p(1-p) U}{2 c}\right\rfloor+1}, \quad\right. \text { and }} \\
& g(c) \equiv \frac{2 \bar{k}^{\mathrm{opt}}\left(\bar{k}^{\mathrm{opt}}+1\right)}{2 \bar{k}^{\mathrm{opt}}+1} c=\left[\left(\left\lfloor\sqrt{\frac{p(1-p) U}{2 c}}\right\rfloor+\frac{1}{2}\right)-\frac{1}{4\left\lfloor\sqrt{\frac{p(1-p) U}{2 c}}\right\rfloor+2}\right] c .
\end{aligned}
$$


We can then define $\Theta(c) \equiv f(c)+g(c)$ to get the representation of $W^{\text {opt }}(c)$ in the corollary. Take any $c<\frac{p(1-p) U}{2}$ for which $\bar{k}^{\text {opt }} \notin \mathbb{Z}_{+}$. There exists $\varepsilon>0$ such that for every $c^{\prime}$ with $\left|c^{\prime}-c\right|<\varepsilon, \bar{k}^{\mathrm{opt}}\left(c^{\prime}\right)=\bar{k}^{\mathrm{opt}}(c){ }^{38}$ Thus, $\Theta(c)$ is differentiable at $c$. Moreover, for any $c<\frac{p(1-p) U}{2}$ such that $\bar{k}^{\mathrm{opt}} \in \mathbb{Z}_{+}, \Theta$ is semidifferentiable. Hence, $\Theta(c)$ is continuous. At any differentiable point $c$ (around which $\bar{k}^{\mathrm{opt}}(c)$ is constant),

$$
\frac{d \Theta(c)}{d c}=\frac{\partial \Theta\left(\bar{k}^{\mathrm{opt}}(c), c\right)}{\partial c}=\frac{2 \bar{k}^{\mathrm{opt}}\left(\bar{k}^{\mathrm{opt}}+1\right)}{2 \bar{k}^{\mathrm{opt}}+1}>0 .
$$

Furthermore, the concavity of $\Theta(c)$ follows from the fact that at any semidifferentiable but not differentiable point $c$,

$$
\frac{d_{-} \Theta(c)}{d c}>\frac{d_{+} \Theta(c)}{d c}
$$

\section{A.2 Proofs regarding discretionary matching}

A.2.1 Players' Markov decision problem In this section, we study stationary* equilibria under several priority protocols. A key first step is to formalize each player's dynamic decision problem, defined by other players' equilibrium strategies, as a Markov decision problem (MDP). We consider here an $H$-square's problem and omit the analogous descriptions for other player types. Fix any priority rule, and take an $H$-square, say player $i$, who arrived in period $t_{0} \geq 1$. Assume that all other players follow a stationary* strategy profile $\Psi_{-i}{ }^{39}$ Player $i$ solves an infinite-horizon dynamic decision problem, defined by $\Psi_{-i}$. For each period $t \geq t_{0}$, let $\theta_{i}^{t}=\left(\mathbf{s}^{t}, q_{i}^{t}\right)$ denote the player's augmented state, where $\mathbf{s}^{t}=\left(s_{H}^{t}, s_{L}^{t}, s_{h}^{t}, s_{l}^{t}\right)$ denotes the state of the market, and $q_{i}^{t}$ denotes player $i$ 's rank among the $H$-squares present. We write $q_{i}^{t}=0$ if player $i$ is matched before period $t$. We denote by $\Theta_{i}$ the set of player $i$ 's possible augmented states. In each period $t \geq t_{0}$, player $i$ chooses a demand $d_{i} \in\{h, l\}$, where $h$ represents a demand for an $h$-round, and $l$ represents a demand for any round. The stage-game payoff $u_{i}\left(d_{i}, \theta_{i}, \Psi_{-i}\right)$ is either a match surplus $\left(U_{H}(h)\right.$ or $\left.U_{H}(l)\right)$, waiting cost $-c$, or 0 (if $q_{i}=0$ ). The initial augmented state is $\theta_{i}^{t_{0}}=\left(\mathbf{s}^{t_{0}}, q_{i}^{t_{0}}\right)$ such that $q_{i}^{t_{0}}=s_{H}^{t_{0}}$ under FIFO and $q_{i}^{t_{0}}=1$ under LIFO. The transition between augmented states is straightforward from our description of the model, hence we omit it here.

A strategy $\sigma_{i}$ is any rule prescribing demands submitted over time. It may entail randomization and it may be history dependent. The payoff for player $i$ from strategy $\sigma_{i}$ is

$$
U_{i}\left(\sigma_{i} ; \theta_{i}, \Psi_{-i}\right) \equiv E_{\sigma_{i}}\left[\sum_{t=t_{0}}^{\infty} u_{i}\left(d_{i}, \theta_{i}^{t}, \Psi_{-i}\right): \theta_{i}^{t_{0}}=\theta_{i}\right] \cdot{ }^{40}
$$

\footnotetext{
${ }^{38}$ We slightly abuse our notation and make the dependence of $\bar{k}^{\text {opt }}$ on the cost $c$ explicit here.

${ }^{39}$ That is, restricting attention to all players but $i$, the strategy profile is stationary* and in particular symmetric.

${ }^{40}$ The expected value exists in $\mathbb{R} \cup\{-\infty\}$. Let $u_{i,+}\left(d_{i}, \theta_{i}, \Psi_{-i}\right)=\max \left\{u_{i}\left(d_{i}, \theta_{i}, \Psi_{-i}\right), 0\right\}$ and $u_{i,-}\left(d_{i}, \theta_{i}, \Psi_{-i}\right)=\max \left\{-u_{i}\left(d_{i}, \theta_{i}, \Psi_{-i}\right), 0\right\}$. Define $U_{i,+}\left(\sigma_{i} ; \theta_{i}^{t_{0}}, \Psi_{-i}\right)=E_{\sigma_{i}}\left[\sum_{t=t_{0}}^{\infty} u_{i,+}\left(d_{i}, \theta_{i}^{t}, \Psi_{-i}\right): \theta_{i}^{t_{0}}\right]$ and
} 
We focus on player $i$ 's Markov random strategies in the sense that a choice in each period is independent of past history. This restriction is without loss of generality since

$$
\sup _{\sigma_{i} \in \Sigma_{i}} U_{i}\left(\sigma_{i} ; \theta_{i}, \Psi_{-i}\right)=\sup _{\sigma_{i} \in \Sigma_{i}^{\mathrm{MR}}} U_{i}\left(\sigma_{i} ; \theta_{i}, \Psi_{-i}\right)
$$

where $\Sigma_{i}$ and $\Sigma_{i}^{\mathrm{MR}}$ denote the set of all strategies and all Markov random strategies, respectively (Proposition 7.1.1 of Puterman 2005). The restriction to Markov random strategies allows us to normalize player $i$ 's arrival time as $t_{0}=0$ and write

$$
U_{i}\left(\sigma_{i} ; \theta_{i}, \Psi_{-i}\right)=E_{\sigma_{i}}\left[\sum_{t=0}^{\infty} u_{i}\left(d_{i}, \theta_{i}^{t}, \Psi_{-i}\right): \theta_{i}^{0}=\theta_{i}\right] \text { for each } \sigma_{i} \in \Sigma_{i}^{\mathrm{MR}} .
$$

We extend player $i$ 's decision problem to a Markov decision problem (MDP) with an arbitrary initial state. That is, an initial state $\theta_{i}^{0}$ can be any element in $\Theta_{i} \subseteq\left\{\left(\mathbf{s}, q_{i}\right) \in\right.$ $\left.\mathbb{Z}_{+}^{5}: s_{H}+s_{L}=s_{h}+s_{l}, 1 \leq q_{i} \leq s_{H}\right\} .{ }^{41}$ A policy $\mu_{i}$ of the (MDP) is any Markovian random rule for choosing demands. A stationary and deterministic policy (SD-policy, for short) applies the same decision rule in every period. We denote an SD-policy by $\psi_{i}: \Theta_{i} \rightarrow$ $\{h, l\}$. The value of a policy $\mu_{i}$, for each initial state $\theta_{i}$, is defined by $v_{i}\left(\mu_{i} ; \theta_{i}, \Psi_{-i}\right) \equiv$ $U_{i}\left(\mu_{i} ; \theta_{i}, \Psi_{-i}\right)$. Last, the value of the (MDP), for each initial state $\theta_{i}$, is $v_{i}^{*}\left(\theta_{i} ; \Psi_{-i}\right) \equiv$ $\sup _{\mu_{i}} v_{i}\left(\mu_{i} ; \theta_{i}, \Psi_{-i}\right)$.

We characterize the value of the (MDP), $v_{i}^{*}\left(\cdot ; \Psi_{-i}\right): \Theta_{i} \rightarrow \mathbb{R} \cup\{-\infty\}$ and find an optimal SD-policy, whose value is equal to $v_{i}^{*}\left(\theta_{i} ; \Psi_{-i}\right)$ for every initial state $\theta_{i}$. An optimal SD-policy of the (MDP) defines a best-response that is a stationary and deterministic strategy for player $i$ given any initial state. A stationary* strategy profile $\Psi=\left(\psi_{H}, \psi_{L}, \psi_{h}, \psi_{l}\right)$ is a stationary* equilibrium if, for every $H$-square (similarly for other types), $\psi_{H}$ is an optimal SD-policy of the (MDP) defined by all other players' equilibrium strategies $\Psi_{-i}$. We use the following definition and theorems from Puterman (2005) that are associated with player $i$ 's problem, but hold for general Markov decision problems.

Definition 3 (Optimality equation; equation (6.2.2) with $\lambda=1$, or equation (7.1.8) of Puterman 2005). We refer to the following system of equations as the optimality equation:

$$
v\left(\theta_{i}\right)=\max _{d \in\{h, l\}}\left[u_{i}\left(d_{i}, \theta_{i}, \Psi_{-i}\right)+\sum_{\theta_{i}^{\prime} \in \Theta_{i}} p\left(\theta_{i}^{\prime} \mid \theta_{i}, d_{i}, \Psi_{-i}\right) v\left(\theta_{i}^{\prime}\right)\right], \quad \text { for all } \theta_{i} \in \Theta_{i} .
$$

Theorem Puterman 1 (Theorem 7.1.3 of Puterman 2005). The value of the (MDP), $v_{i}^{*}\left(\cdot ; \Psi_{-i}\right)$, is a solution of the optimality equation.

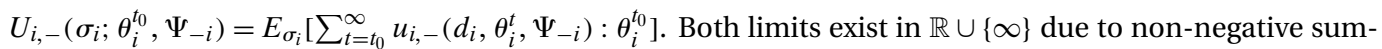
mands, and $U_{i,+}\left(\sigma_{i} ; \theta_{i}^{t_{0}}, \Psi_{-i}\right)$ is finite as it is bounded above by $U_{H}(h)$. It follows that $U_{i}\left(\sigma_{i} ; \theta_{i}^{t_{0}}, \Psi_{-i}\right)=$ $U_{i,+}\left(\sigma_{i} ; \theta_{i}^{t_{0}}, \Psi_{-i}\right)-U_{i,-}\left(\sigma_{i} ; \theta_{i}^{t_{0}}, \Psi_{-i}\right)$ exists in $\mathbb{R} \cup\{-\infty\}$.

${ }^{41}$ As mentioned, the initial condition $\theta_{i}^{0}=\left(\mathbf{s}^{0}, q_{i}^{0}\right)$ should satisfy $q_{i}^{0}=s_{H}^{0}$ under FIFO and $q_{i}^{0}=1$ under LIFO. We remove such restrictions in the (MDP). 
Theorem Puterman 2 (Theorem 7.2.5 (a) of Puterman 2005). A policy $\mu_{i}^{*}$ is optimal if and only if the value of the policy $v_{i}^{*}\left(\cdot ; \mu_{i}^{*}, \Psi_{-i}\right): \Theta_{i} \rightarrow R \cup\{-\infty\}$ is a solution of the optimality equation.

Note that the value of the (MDP) is not a unique solution of the optimality equation. For example, we can add a constant to the value and find another solution. This nonuniqueness is a consequence of there not being discounting in our model. Finally, the state space $\Theta_{i}$ for player $i$ can be finite under some stationary* strategy-profile $\Psi_{-i}$ chosen by other players. We have

Theorem Puterman 3 (Theorem 7.1.9 of Puterman 2005). If $\Theta_{i}$ is finite, then there exists an optimal SD-policy.

A.2.2 Proofs regarding stationary* equilibria under FIFO The following Lemmas A2 and $\mathrm{A} 3$ are employed in the proofs of Lemmas 1 and 2.

Lemma A2. Under FIFO, if $\Psi^{*}=\left(\psi_{H}^{*}, \psi_{L}^{*}, \psi_{h}^{*}, \psi_{l}^{*}\right)$ is a stationary* equilibrium, then

$$
\psi_{H}^{*}(\mathbf{s}, q)= \begin{cases}h \text { orl } & \text { if } q \leq s_{h}, \\ h & \text { if } 1 \leq q-s_{h} \leq \bar{k}^{\text {fifo }}, \\ l & \text { otherwise }\end{cases}
$$

where

$$
\bar{k}^{\text {fifo }} \equiv\left\lfloor\frac{p\left(U_{H}(h)-U_{H}(l)\right)}{c}\right\rfloor=\left\lfloor\frac{p\left(U_{h}(H)-U_{h}(L)\right)}{c}\right\rfloor .
$$

An analogous claim holds for $h$-rounds.

Proof of Lemma A2. We show that if $\Psi^{*}$ is a stationary* equilibrium, for any augmented state $\theta_{i}=\left(\mathbf{s}, q_{i}\right)$ for player $i$, who is an $H$-square, we have

$$
\psi_{H}^{*}\left(\theta_{i}\right)= \begin{cases}h & \text { if } 1 \leq q_{i}-s_{h} \leq \bar{k}^{\text {fifo }} \\ l & \text { if } q_{i}-s_{h}>\bar{k}^{\text {fifo }}\end{cases}
$$

The proof is by induction. Take any stationary* strategy-profile $\Psi=\left(\psi_{H}, \psi_{L}, \psi_{h}, \psi_{l}\right)$. First, we characterize the equilibrium behavior of player $i$, an $H$-square, when she finds no available $h$-round in a period, and is positioned in the queue so that she is to become first in line if she stays for an additional period. Formally, $i$ 's augmented state in period $t_{0}$ satisfies $q_{i}^{t_{0}}=s_{h}^{t_{0}}+1$. Indeed, in period $t_{0}$, player $i$ finds no available $h$-round (i.e., $q_{i}^{t_{0}}>s_{h}^{t_{0}}$ ) and, if she is not matched, she becomes the first $H$-square in the queue (i.e., $q_{i}^{t_{0}}-s_{h}^{t_{0}}=1$ ). In finding a dynamic best-response from period $t_{0}$ onward, it is without loss of generality to restrict attention to player $i$ 's Markov random strategies. Once we restrict attention to Markov random strategies, we can normalize $t_{0}=0$. Player $i$ solves 
the following problem:

$$
v_{i}^{*}\left(\theta_{i} ; \Psi_{-i}\right) \equiv \sup _{\sigma_{i} \in \Sigma_{i}^{\mathrm{MR}}} E_{\sigma_{i}}\left[\sum_{t=0}^{\infty} u_{i}\left(d_{i}, \theta_{i}^{t}, \Psi_{-i}\right): \theta_{i}^{0}=\theta_{i}\right] .
$$

Since $s_{l}^{0} \geq q_{i}^{0}+s_{L}^{0}-s_{h}^{0}>0$, we know that at least one $l$-round is available in period $t_{0}=0$. Also, the first $h$-round to arrive at the market will be available to match with player $i$, but it takes, in expectation, $1 / p$ periods until such $h$-round arrives. Therefore, we have $v_{i}^{*}\left(\theta_{i} ; \Psi_{-i}\right)=\max \left\{U_{H}(l), U_{H}(h)-\frac{c}{p}\right\}$. Hence, if $\psi_{H}^{*}$ is part of a stationary* equilibrium, it must be that

$$
\psi_{H}^{*}\left(\theta_{i}\right)= \begin{cases}h & \text { if } q_{i}-s_{h}=1 \leq \bar{k}^{\text {fifo }} \\ l & \text { if } q_{i}-s_{h}=1>\bar{k}^{\text {fifo }} .\end{cases}
$$

That is, (9) holds for every $\theta_{i}=\left(\mathbf{s}, q_{i}\right)$ with $q_{i}-s_{h}=1$.

Next, we complete the induction. Take any $k \in \mathbb{Z}_{++}$and a stationary* strategy-profile $\Psi$ such that $\psi_{H}$ satisfies (9) for every augmented state $\theta=(\mathbf{s}, q)$ with $q-s_{h} \leq k$. Consider any $H$-square, say player $i$, whose augmented state in period 0 (normalized, as above) satisfies $q_{i}^{0}=s_{h}^{0}+(k+1)$. Assume that every other $H$-square, say player $j$ with $q_{j}^{0} \leq$ $s_{h}^{0}+k<q_{i}^{0}$, plays $\psi_{H}$. Given that each player's rank in the queue only improves over time, the first $\min \left\{k, \bar{k}^{\mathrm{fifo}}\right\}$ arriving $h$-rounds in the future are not available for player $i$, but the next arriving $h$-round will be. In expectation, it takes $\frac{\min \left\{k,,^{\text {fifo }}\right\}+1}{p}$ periods until an $h$-round becomes available for player $i$. As such,

$$
\begin{aligned}
v_{i}^{*}\left(\theta_{i} ; \Psi_{-i}\right) & =\max \left\{U_{H}(l), U_{H}(h)-\frac{\left(\min \left\{k, \bar{k}^{\text {fifo }}\right\}+1\right) c}{p}\right\} \\
& =\max \left\{U_{H}(l), U_{H}(h)-\frac{(k+1) c}{p}\right\},
\end{aligned}
$$

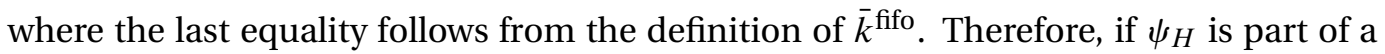
stationary* equilibrium, $\psi_{H}\left(\theta_{i}\right)$ must satisfy (9) for any augmented state $\theta_{i}=\left(\mathbf{s}, q_{i}\right)$ with $q_{i}-s_{h}=k+1$.

Lemma A3. There exists a stationary* equilibrium $\Psi^{*}=\left(\psi_{H}^{*}, \psi_{L}^{*}, \psi_{h}^{*}, \psi_{l}^{*}\right)$ such that (a) $\psi_{H}^{*}$ (and $\psi_{h}^{*}$ ) satisfies (8) (with an analogous condition for h-rounds), and (b) $\psi_{l}^{*}\left(s, s_{l}\right)=L$ and $\psi_{L}^{*}\left(s, s_{L}\right)=l$, whenever $s_{L}>0$ and $s_{l}>0$.

Proof of Lemma A3. We start with the analysis of the $H$-squares' decisions. Take any stationary* strategy-profile $\Psi=\left(\psi_{H}, \psi_{L}, \psi_{h}, \psi_{l}\right)$ that satisfies conditions (a) and (b) in the claim. We prove that $\psi_{H}$ is a best-response for an $H$-square, say player $i$, regardless of her initial augmented state. Let $\Theta_{H}$ be the set of all possible augmented states for 
player $i$, conditional on $\Psi_{-i}$. That is, ${ }^{42}$

$$
\Theta_{H} \equiv\left\{(\mathbf{s}, q) \in \mathbb{Z}_{+}^{5}:-\bar{k}^{\text {fifo }}-1 \leq s_{H h} \leq \bar{k}^{\text {fifo }}+2, q_{i} \leq s_{H}\right\} .
$$

We extend player $i$ 's decision problem as a (MDP) with an arbitrary initial state (ignoring the fact that her initial state in the discretionary matching is $q_{i}=s_{H}$ ). That is, player $i$ 's (MDP) is

$$
v_{i}^{*}\left(\theta_{i} ; \Psi_{-i}\right) \equiv \sup _{\mu_{i} \in \Sigma_{i}^{\mathrm{MR}}} v_{i}\left(\mu_{i} ; \theta_{i}, \Psi_{-i}\right), \quad \text { for all } \theta_{i} \in \Theta_{H},
$$

where

$$
v_{i}\left(\theta_{i} ; \mu_{i}, \Psi_{-i}\right) \equiv U_{i}\left(\mu_{i} ; \theta_{i}, \Psi_{-i}\right) \equiv E_{\mu_{i}}\left[\sum_{t=0}^{\infty} u_{i}\left(d_{i}, \theta_{i}^{t}, \Psi_{-i}\right): \theta_{i}^{0}=\theta_{i}\right] .
$$

If player $i$ follows the SD-policy $\psi_{H}$ that satisfies (8), then for $\theta_{i}=\left(\mathbf{s}, q_{i}\right)$,

$$
\begin{aligned}
v_{i}\left(\theta_{i} ; \psi_{H}, \Psi_{-i}\right) & =E\left[\sum_{t=0}^{\infty} u_{i}\left(\psi_{H}\left(\theta_{i}^{t}\right), \theta_{i}^{t}, \Psi_{-i}\right): \theta_{i}^{0}=\theta_{i}\right] \\
& = \begin{cases}U_{H}(h) & \text { if } q_{i} \leq s_{h} \\
U_{H}(h)-\frac{\left(q_{i}-s_{h}\right) c}{p} & \text { if } 1 \leq q_{i}-s_{h} \leq \bar{k}^{\text {fifo }} \\
U_{H}(l) & \text { otherwise. }\end{cases}
\end{aligned}
$$

From the construction of $\psi_{H}$ in the proof of Lemma A2, it is easy to verify that $v_{i}\left(\cdot ; \psi_{H}, \Psi_{-i}\right): \Theta_{H} \rightarrow \mathbb{R} \cup\{-\infty\}$ solves the optimality equation. Thus, by Theorem Puterman 2, $\psi_{H}$ is an optimal SD-policy of player $i$ 's (MDP). In particular, each $H$-square is best-responding by playing $\psi_{H}$, regardless of her initial augmented state. Next, we consider $l$-rounds' decisions. Let $\Theta_{l}$ denote the set of all possible augmented states that an $l$-round may experience:

$$
\Theta_{l} \equiv\left\{(\mathbf{s}, q) \in \mathbb{Z}_{+}^{5}: s_{H}+s_{L}=s_{h}+s_{l}, q \leq s_{l}\right\},
$$

where $q=0$ represents the augmented state after the player is matched. We take $\psi_{H}$ and $\psi_{h}$ satisfying condition (a) in Lemma A3. We want to construct an SD-strategy $\psi_{l}$ : $\Theta_{l} \rightarrow\{H, L\}$ (and $\psi_{L}: \Theta_{L} \rightarrow\{h, l\}$, whose analogous construction we omit) such that $\Psi=\left(\psi_{H}, \psi_{L}, \psi_{h}, \psi_{l}\right)$ constitutes a stationary* equilibrium. The following assumption on $\psi_{l}$ (and $\psi_{L}$ ) will be useful for our construction.

Assumption Al. For any $\left(s, s_{l}\right) \in \Theta_{l},\left(s, s_{L}\right) \in \Theta_{L}$ with $s_{l}>0$ and $s_{L}>0$, (1) $\psi_{l}\left(s, s_{l}\right)=$ $\psi_{l}\left(s, s_{l}-1\right)=\cdots=\psi_{l}\left(s, 1+s_{H h}^{+}\right)=L ;(2) \psi_{L}\left(s, s_{L}\right)=\psi_{L}\left(s, s_{L}-1\right)=\cdots=\psi_{L}(s, 1+$ $\left.s_{H h}^{-}\right)=l$, where $s_{H h}^{+}=\max \left\{s_{H h}, 0\right\}$ and $s_{H h}^{-}=-\min \left\{s_{H h}, 0\right\}$.

\footnotetext{
${ }^{42}$ Recall that $q_{i}^{t}=0$ for any period $t$ after player $i$ matches. If $q_{i}^{t}=0$, player $i$ 's stage-game payoff is $u_{i}^{t}=0$. Moreover, $s_{H h}=\bar{k}^{\text {fifo }}+2$ can occur, if player $i$ deviates from $\psi_{H}$. For example, player $i$ may arrive at the market with a rank $q_{i}=s_{H h}=\bar{k}^{\text {fifo }}+1$. If she deviates from $\psi_{H}$ by demanding $h$, then $s_{H h}$ can be $\bar{k}^{\text {fifo }}+2$ in the following period due to an additional arrival of an $H$-square.
} 
Note that Assumption A1 is consistent with condition (b) in Lemma A3. The construction of $\psi_{l}$ that follows will guarantee that $\psi_{l}$ is a best response for an $l$-round in any period and with any initial augmented state, if all other players satisfy Assumption A1. We will then justify Assumption Al as describing best-response strategies. Take any $l$-round, say player $i$, and any stationary* strategy-profile $\Psi_{-i}$ such that $\psi_{H}$ and $\psi_{h}$ satisfy (8) and Assumption Al holds. As argued before, there is no period in which both $H$-squares and $h$-rounds wait at the market. Therefore, for any $t$, the stock $\mathbf{k}^{t} \equiv\left(k_{H}^{t}, k_{L}^{t}, k_{h}^{t}, k_{l}^{t}\right)$ satisfies $k_{H}^{t} k_{h}^{t}=0$ and $-\bar{k}^{\text {fifo }} \leq k_{H h}^{t} \leq \bar{k}^{\text {fifo }}$. In addition, by Assumption Al, there is no period in which at least two $L$-squares and two $l$-rounds wait by demanding $h$-rounds and $H$-squares, respectively. We characterize the set of augmented states for player $i$, which we denote by $\Theta_{l}^{\prime} \subseteq \Theta_{l}$. Let $K \subseteq \mathbb{Z}_{+}^{4}$ denote the set of possible states at the end of each period. That is,

$$
\mathbf{k} \equiv\left(k_{H}, k_{L}, k_{h}, k_{l}\right) \in K \Longleftrightarrow \begin{gathered}
\text { (i) } k_{H}+k_{L}=k_{h}+k_{l}, \\
\text { (ii) } k_{H} k_{h}=0, \\
\text { (iii) }-\bar{k}^{\text {fifo }} \leq k_{H h} \leq \bar{k}^{\text {fifo }}, \\
\text { (iv) } k_{H h} \geq 0 \Longrightarrow k_{L} \leq 1 \text {, and } k_{H h} \leq 0 \Longrightarrow k_{l} \leq 1 .
\end{gathered}
$$

Then $\Theta_{l}^{\prime}$ is a subset of $\Theta_{l}$ such that

$$
\begin{aligned}
(\mathbf{s}, q) \in \Theta_{l}^{\prime} \Longleftrightarrow & (\exists \mathbf{k} \in K) \quad \text { s.t. } \mathbf{s}-\mathbf{k} \in\{(1,0,1,0),(0,1,0,1),(1,0,0,1),(0,1,1,0)\} \\
& \text { and } q \leq s_{l} .
\end{aligned}
$$

It is clear that $\Theta_{l}^{\prime}$ is finite, and for any $(\mathbf{s}, q) \in \Theta_{l}^{\prime}$, we have $0 \leq q \leq \bar{k}^{\text {fifo }}+2$. It is sufficient to define $\psi_{l}$ over augmented states in $\Theta_{l}^{\prime}$ only, as an augmented state $(\mathbf{s}, q) \notin \Theta_{l}^{\prime}$ never occurs. Under the FIFO protocol, the ranking of an $l$-round, such as our player $i$, improves as she waits in the market. Thus, player $i$ 's continuation payoff from the (MDP) after her rank becomes 1 is independent of her actions in a state with a rank lower than 1. For each possible ranking of an $l$-round, $q \in\left\{1,2, \ldots, \bar{k}^{\text {fifo }}+2\right\}$, let $\Theta_{l, q}^{\prime}$ be the set of augmented states with rank $q$ (i.e., $\left.\Theta_{l, q}^{\prime} \equiv\left\{\mathbf{s} \mid(\mathbf{s}, q) \in \Theta_{l}^{\prime}\right\}\right)$. We construct $\psi_{l, q}: \Theta_{l, q}^{\prime} \rightarrow\{H, L\}$ sequentially from $q=1$ to $q=\bar{k}^{\text {fifo }}+2$, and define $\psi_{l}: \Theta_{l}^{\prime} \rightarrow\{H, L\}$ as $\psi_{l}(\mathbf{s}, q) \equiv \psi_{l, q}(\mathbf{s}, q)$. In the construction, we will guarantee that $\psi_{l}$ constitutes a best response for an $l$-round, taking as given $\psi_{H}, \psi_{h}$, and Assumption Al applied to all other players. The proof is inductive with the following induction hypothesis.

Induction hypothesis. There exists $\psi_{l, q}: \Theta_{l, q}^{\prime} \rightarrow\{H, L\}$ for $q \leq \bar{k}^{\text {fifo }}+2$ such that:

(i) $\psi_{l, \leq q}=\left(\psi_{l, q}, \psi_{l, q-1}, \ldots, \psi_{l, 1}\right)$ is a optimal $S D$-policy for player $i$, and

(ii) the maximal total expected payoff for player $i$, given any $\theta_{i}=\left(s, q_{i}\right) \in \Theta_{l, q}^{\prime}$, is

$$
v_{i}^{*}\left(\theta_{i}\right) \leq \max \left\{U_{l}(L), U_{l}(H)-\frac{\left(\bar{k}^{\text {fifo }}-s_{H h}+q_{i}\right) c}{p}\right\} .
$$


Step 1: Construction of $\psi_{l, 1}$

Consider an $l$-round, say our player $i$, who is the first in the queue at some period. Player $i$ solves a dynamic decision problem, defined by $\psi_{H}$, $\psi_{h}$, and Assumption Al (applied to other players' strategies). We extend player $i$ 's decision problem as a (MDP) with an arbitrary initial state $\theta_{i} \in \Theta_{l, 1}^{\prime}$. Let $v^{*}\left(\theta_{i}\right)$ denote the maximal expected total payoff for player $i$ with an initial augmented state $\theta_{i}$. Theorem Puterman 3 guarantees that there exists an optimal SD-policy. Moreover, any policy whose values solve the optimality equation is optimal by Theorem Puterman 2, which allows us to choose a particular optimal SD-policy $\psi_{l, 1}$ consistent with Assumption Al. To proceed with the construction, we show the following claims.

Claim 1. For any $\theta_{i} \in(s, 1) \in \Theta_{l, 1}$, we have $v^{*}\left(\theta_{i}\right) \leq \max \left\{U_{l}(L), U_{l}(H)-\frac{\left(\bar{k}^{\mathrm{fifo}}-s_{H h}+1\right) c}{p}\right\}$.

Proof of Claim 1. Take an $l$-round, say player $i$, who is the first in the queue for $l$ rounds in some period, which we normalize to be $t_{0}=0$, and augmented state $\left(\mathbf{s}^{0}, 1\right) \in$ $\Theta_{l, 1}^{\prime}$. Given $\psi_{H}$, to match with an $H$-square, player $i$ must wait for at least $\bar{k}^{\text {fifo }}-s_{H h}^{0}+1$ additional arrivals of $H$-squares. Consider now the following optimal stopping problem:

[P] A boy (l) stands under an apple tree and holds a banana. In each period, one apple falls from the tree with probability $p$. The first $\bar{k}^{*}\left(\equiv \bar{k}^{\text {fifo }}-s_{H h}^{0}\right)$ apples should be handed over to the owner of the tree. The boy can consume exactly one piece of fruit, either an apple or a banana. He prefers an apple, with payoff $U_{l}(H)$, to the banana, with payoff $U_{l}(L)$. Thus, while he can consume the banana and walk away with $U_{l}(L)$ in any period, he may want to wait for falling apples. He incurs a cost c for each period of waiting without consuming any fruit.

Let $\Theta_{(P)} \equiv\left\{0,1, \ldots, \bar{k}^{*}+1\right\} \cup\{\Delta\}$ denote the state space of $[\mathrm{P}]$, where $\Delta$ denotes the (absorbing) state after the boy consumes a piece of fruit. In each period $t$ and state $\theta_{(P)}^{t} \in \Theta_{(P)} \backslash\{\Delta\}$, the boy chooses a demand $d \in\{H, L\}$. The stage payoff from demand $H$ is either $U_{l}(H)$ in state $\theta_{(P)}^{t}=\bar{k}^{*}+1$, or $-c$ in any other state in $\Theta_{(P)} \backslash\{\Delta\}$. The stage payoff from demand $L$ is $U_{l}(L)$ in any state in $\Theta_{(P)} \backslash\{\Delta\}$. In state $\Delta$ (i.e., after consuming a piece of fruit), the boy gets zero stage payoff forever. The value of $[\mathrm{P}]$ with an arbitrary initial state $\theta \in \Theta_{(P)}$ is ${ }^{43}$

$$
v_{(P)}^{*}(\theta) \equiv \sup _{\mu} E_{\mu}\left[\sum_{t=0}^{\infty} u\left(d, \theta^{t}\right): \theta^{0}=\theta\right] .
$$

It is clear from the description of $[\mathrm{P}]$ that $v_{(P)}^{*}(0)$ constitutes an upper bound for the maximal expected total payoff of player $i$ (i.e., $v^{*}\left(\theta_{i}\right)$ ). In fact, unlike player $i$, the boy in [P] can always consume a banana and walk away. Also, while player $i$ must wait for at least $\bar{k}^{*}+1$ arrivals of $H$-squares to match with an $H$-square, the boy in [P] is guaranteed to get the $\left(\bar{k}^{*}+1\right)$-th falling apple. As such, to prove the claim, it is sufficient to show that

$$
v_{(P)}^{*}(0) \leq \max \left\{U_{l}(L), U_{l}(H)-\frac{\left(\bar{k}^{\text {fifo }}-s_{H h}^{0}+1\right) c}{p}\right\} .
$$

\footnotetext{
${ }^{43}$ The limit exists, because P is a positive bounded problem (see p. 279 of Puterman 2005).
} 
Let

$$
\bar{k}^{* *} \equiv\left\lfloor\frac{p\left(U_{l}(H)-U_{l}(L)\right)}{c}\right\rfloor \leq \bar{k}^{\text {fifo }} .
$$

(i) Suppose that $\bar{k}^{*}<\bar{k}^{* *}$. Then, compared to consuming a banana immediately, it is weakly more profitable to wait until $\bar{k}^{*}+1=\bar{k}^{\text {fifo }}-s_{H h}^{0}+1\left(\leq \bar{k}^{* *}\right)$ apples fall. Once the boy decides to wait, he will continue to wait until he obtains an apple. Thus,

$$
v_{(P)}^{*}(0)=U_{l}(H)-\frac{\left(\bar{k}^{*}+1\right) c}{p}=U_{l}(H)-\frac{\left(\bar{k}^{\text {fifo }}-s_{H h}^{0}+1\right) c}{p} .
$$

(ii) Suppose that $\bar{k}^{*}=\bar{k}^{* *}$. As $v_{(P)}^{*}(\cdot)$ solves the optimality equation, we have

$$
v_{(P)}^{*}(0)=\max \left\{U_{l}(L),-c+p\left(v_{(P)}^{*}(1)\right)+(1-p)\left(v_{(P)}^{*}(0)\right)\right\} .
$$

Suppose, toward a contradiction, that

$$
v_{(P)}^{*}(0)=-c+p\left(v_{(P)}^{*}(1)\right)+(1-p) v_{(P)}^{*}(0)>U_{l}(L) .
$$

Then

$$
v_{(P)}^{*}(0)=v_{(P)}^{*}(1)-\frac{c}{p}=\left(U_{l}(H)-\frac{\bar{k}^{*} c}{p}\right)-\frac{c}{p}=U_{l}(H)-\frac{\left(\bar{k}^{* *}+1\right) c}{p}>U_{l}(L),
$$

where the second equality follows from case (i) above (after the first apple falls, the boy needs to hand over only $\bar{k}^{*}-1\left(<\bar{k}^{* *}\right)$ additional apples to the owner). Notice that the last inequality contradicts the definition of $\bar{k}^{* *}$. Therefore, $v_{(P)}^{*}(0) \leq U_{l}(L)$.

(iii) Suppose that $\bar{k}^{*}>\bar{k}^{* *}$. More apples should be handed over to the owner than in the previous case, so $v_{(P)}^{*}(0) \leq U_{l}(L)$.

This concludes the proof of Claim 1 .

CLAIM 2. There exists an optimal SD-policy $\psi_{l, 1}: \Theta_{l, 1}^{\prime} \rightarrow\{H, L\}$ of the (MDP) for player $i$ such that $\psi_{l, 1}\left(\theta_{i}\right)=L$, for all $\theta_{i}=(s, 1) \in \Theta_{l, 1}^{\prime}$ with $s_{H h}<1$.

Proof of Claim 2. Let $\psi_{l, 1}: \Theta_{l, 1}^{\prime} \rightarrow\{H, L\}$ such that

$$
\psi_{l, 1}\left(\theta_{i}\right)= \begin{cases}H & \text { if } v^{*}\left(\theta_{i}\right)>U_{l}(L), \\ L & \text { if } v^{*}\left(\theta_{i}\right) \leq U_{l}(L) .\end{cases}
$$

Then $v_{i}\left(\cdot ; \psi_{l, 1}\right): \Theta_{l, 1}^{\prime} \rightarrow \mathbb{R} \cup\{-\infty\}$ is a solution of the optimality equation of player $i$ 's (MDP). It follows from Theorem Puterman 2 that the SD-policy $\psi_{l, 1}$ is optimal. By Claim 1, for any $\theta_{i}=(\mathbf{s}, 1) \in \Theta_{l, 1}^{\prime}$ with $s_{H h}<1$,

$$
v_{i}^{*}\left(\theta_{i}\right) \leq \max \left\{U_{l}(L), U_{l}(H)-\frac{\left(\bar{k}^{\mathrm{fifo}}+1\right) c}{p}\right\}=U_{l}(L),
$$

so that $\psi_{l, 1}\left(\theta_{i}\right)=L$. This concludes the proof of Claim 2 . 
Step 2: Construction of $\psi_{l, q+1}$ given $\left(\psi_{l, 1}, \psi_{l, 2}, \ldots, \psi_{l, q}\right)$

Fix $q \in\left\{1,2, \ldots, \bar{k}^{\text {fifo }}+1\right\}$. For an $l$-round, say player $i$, who enters as $q$ th in line, we extend the player's dynamic decision problem as a (MDP) with an arbitrary initial augmented-state set $\Theta_{l, \leq q}^{\prime} \equiv \bigcup_{q^{\prime} \leq q} \Theta_{l, q^{\prime}}^{\prime}$. Note that the (MDP) for player $i$ is defined by $\psi_{H}, \psi_{h}, \psi_{l,<q} \equiv\left(\psi_{l, q-1}, \psi_{l, q-2}, \ldots, \psi_{l, 1}\right), \psi_{L,<q} \equiv\left(\psi_{L, q-1}, \psi_{L, q-2}, \ldots, \psi_{L, 1}\right)$, and by Assumption Al applied to other players' strategies.

Now, consider an $l$-round, say player $j$, who is $(q+1)$-th in line at some period $t_{0}=0$ (normalized). Player $j$ solves a dynamic decision problem. As before, we extend player $j$ 's problem as a (MDP) with an arbitrary initial augmented state in the set $\Theta_{l, \leq q+1}^{\prime} \equiv \bigcup_{q^{\prime} \leq q+1} \Theta_{l, q^{\prime}}^{\prime}$. Note that player $j^{\prime}$ (MDP) is defined by $\psi_{H}, \psi_{h}, \psi_{L, \leq q}, \psi_{l, \leq q}$, and by Assumption Al applied to other players' strategies. As the set of augmented states for player $j$ is still finite, there exists an optimal SD-policy (see Theorem Puterman 3). Moreover, any policy whose values solve the optimality equation is optimal (see Theorem Puterman 2). In particular, it is optimal for player $j$ to follow any optimal SD-policy of his (MDP) until his rank becomes $q$, after which he switches to any optimal policy of a (MDP) for an $l$-round who enters as $q$ th in line. Thus, to find an optimal policy for player $j$, it is sufficient to find a function $\psi_{l, q+1}: \Theta_{l, q+1}^{\prime} \rightarrow\{H, L\}$ that is consistent with Assumption A1.

Claim 3. For any $\theta_{j}=(s, q+1) \in \Theta_{l, q+1}^{\prime}$,

$$
v_{j}^{*}\left(\theta_{j}\right) \leq \max \left\{U_{l}(L), U_{l}(H)-\frac{\left(\bar{k}^{\text {fifo }}-s_{H h}+q+1\right) c}{p}\right\} .
$$

Proof of Claim 3. Take an $l$-round, say player $j$, who is $(q+1)$-th in the queue of $l$ rounds in period $t_{0}=0$ (normalized), and any augmented state $\left(\mathbf{s}^{0}, q+1\right) \in \Theta_{l, q+1}^{\prime}$. Two observations will be useful:

1. In any augmented state $\theta_{j}=(\mathbf{s}, q+1) \in \Theta_{l, q+1}^{\prime}$, if there exists any $q^{\prime}<q+1$ such that $\psi_{\left(\mathbf{s}, q^{\prime}\right)}=L$, the maximum expected continuation payoff for player $j$ is at most $U_{l}(L)$.

2. In any augmented state $\theta_{j}=(\mathbf{s}, q+1)$ with $s_{H h}=\bar{k}^{\text {fifo }}+1$, the first $l$-round in the queue matches with an $H$-square. Thus, the maximum expected continuation payoff for player $j$ (i.e., $v_{j}^{*}\left(\theta_{j}\right)$ ) equals $v_{j}^{*}\left(\mathbf{s}^{\prime}, q\right)$, where $\mathbf{s}^{\prime}$ denotes the augmented state after matching the first $l$-round with an $H$-square. ${ }^{44}$ As $s_{H h}^{\prime}=\bar{k}^{\text {fifo }}$, by the induction hypothesis holding up to $q$,

$$
v_{j}^{*}\left(\theta_{j}\right)=v_{j}^{*}\left(\mathbf{s}^{\prime}, q\right) \leq \max \left\{U_{l}(L), U_{l}(H)-\frac{q c}{p}\right\} .
$$

Player $j$ either matches with an $L$-square and receives $U_{l}(L)$ while his rank is $q+1$ or has a corresponding augmented state at some period before matching. Moreover, starting from an arbitrary initial augmented state $\theta_{j}^{0}=\left(\mathbf{s}^{0}, q+1\right)$, the second case occurs

${ }^{44}$ That is, $\left(s_{H}^{\prime}, s_{L}^{\prime}, s_{h}^{\prime}, s_{l}^{\prime}\right)=\left(s_{H}, s_{L}, s_{h}, s_{l}\right)-(1,0,0,1)$. 
only after at least $\bar{k}^{\text {fifo }}-s_{H h}^{0}+1$ arrivals of $H$-squares. Consider the following optimal stopping problem:

[P'] A boy (l) stands under an apple tree and holds a banana. In each period, one apple falls from the tree with probability $p$. The first $\bar{k}^{*}\left(\equiv \bar{k}^{\text {fifo }}-s_{H h}^{0}\right)$ falling apples should be handed over to the owner of the apple tree. The boy can consume exactly one piece of fruit, either an apple or the banana. He (weakly) prefers an apple, with payoff $U_{l}^{\prime}(H) \equiv \max \left\{U_{l}(L), U_{l}(H)-\right.$ $\left.\frac{q c}{p}\right\}$, to the banana, with payoff $U_{l}(L)$. Thus, while he can consume the banana and walk away in any period, he may want to wait for falling apples. He incurs a cost c for each period of waiting without consuming any fruit.

Similar to the proof of Claim 1, let $\Theta_{\left(P^{\prime}\right)} \equiv\left\{0,1, \ldots, \bar{k}^{*}+1\right\} \cup\{\Delta\}$ denote the state space of [P'], where $\Delta$ denotes the (absorbing) state after the boy consumes a fruit. The value of [P'] with an arbitrary initial state $\theta \in \Theta_{\left(P^{\prime}\right)}$ exists (by similar arguments to those used for the existence of the value of $[\mathrm{P}])$. The value of $\left[\mathrm{P}^{\prime}\right]$ with the initial condition 0 , denoted by $v_{\left(P^{\prime}\right)}^{*}(0)$, is an upper bound of the maximal expected payoff for player $j$. Unlike player $j$, the boy in [P'] can always consume a banana and walk away. While player $j$ must wait for at least $\bar{k}^{*}+1$ arrivals of $H$-squares to get an expected continuation payoff of $U_{l}^{\prime}(H)$, the boy in [P'] is guaranteed to get $U_{l}^{\prime}(H)$ after $\bar{k}^{*}+1$ falling apples. As such, it is sufficient to prove that

$$
v_{\left(P^{\prime}\right)}^{*}(0) \leq \max \left\{U_{l}(L), U_{l}(H)-\frac{\bar{k}^{\mathrm{fifo}}-s_{H h}^{0}+q+1}{p}\right\} .
$$

Let $\bar{k}^{* *} \equiv\left\lfloor\frac{p\left(U_{l}^{\prime}(H)-U_{l}(L)\right)}{c}\right\rfloor \leq \bar{k}^{\text {fifo }}$. As in the proof of Claim 1, we consider three cases:

(i) Suppose that $\bar{k}^{*}<\bar{k}^{* *}$. Compared to consuming a banana immediately, it is weakly more profitable to wait until $\bar{k}^{*}+1=\bar{k}^{\text {fifo }}-s_{H h}^{0}+1\left(\leq \bar{k}^{* *}\right)$ apples fall. Once the boy waits, he will continue to wait until he obtains an apple. Thus,

$$
\begin{aligned}
v_{\left(P^{\prime}\right)}^{*}(0) & =U_{l}^{\prime}(H)-\frac{\left(\bar{k}^{*}+1\right) c}{p}=U_{l}^{\prime}(H)-\frac{\left(\bar{k}^{\text {fifo }}-s_{H h}^{0}+1\right) c}{p} \\
& \leq \max \left\{U_{l}(L), U_{l}(H)-\frac{\left(\bar{k}^{\text {fifo }}-s_{H h}^{0}+q+1\right) c}{p}\right\} .
\end{aligned}
$$

(ii) Suppose that $\bar{k}^{*}=\bar{k}^{* *}$. As $v_{\left(P^{\prime}\right)}^{*}(\cdot)$ solves the optimality equation (see Theorem Puterman 1),

$$
v_{\left(P^{\prime}\right)}^{*}(0)=\max \left\{U_{l}(L),-c+p\left(v_{\left(P^{\prime}\right)}^{*}(1)\right)+(1-p)\left(v_{\left(P^{\prime}\right)}^{*}(0)\right)\right\} .
$$

Assume, toward a contradiction, that

$$
v_{\left(P^{\prime}\right)}^{*}(0)=-c+p\left(v_{\left(P^{\prime}\right)}^{*}(1)\right)+(1-p)\left(v_{\left(P^{\prime}\right)}^{*}(0)\right)>U_{l}(L) .
$$

Then

$$
v_{\left(P^{\prime}\right)}^{*}(0)=v_{\left(P^{\prime}\right)}^{*}(1)-\frac{c}{p}=U_{l}^{\prime}(H)-\frac{\left(\bar{k}^{*}+1\right) c}{p}=U_{l}^{\prime}(H)-\frac{\left(\bar{k}^{* *}+1\right) c}{p}>U_{l}(L),
$$


where the second equality is from case (i). After the first falling apple, the boy needs to hand over only $\bar{k}^{*}-1\left(<\bar{k}^{* *}\right)$ additional apples to the owner. The last inequality contradicts the definition of $\bar{k}^{* *}$. Therefore, $v_{\left(P^{\prime}\right)}^{*}(0) \leq U_{l}(L)$.

(iii) Suppose that $\bar{k}^{*}>\bar{k}^{* *}$. More apples should be handed over to the owner than in the previous case, so $v_{\left(P^{\prime}\right)}^{*}(0) \leq U_{l}(L)$.

This concludes the proof of Claim 3 .

Claim 4. There exists $\psi_{l, q+1}: \Theta_{l, q+1}^{\prime} \rightarrow\{H, L\}$ with

$$
\psi_{l, q+1}\left(\theta_{j}\right)=L, \quad \text { for all } \theta_{j}=(\mathbf{s}, q+1) \in \Theta_{l, q+1}^{\prime} \text { with } s_{H h}<q+1,
$$

such that $\psi_{l, \leq q+1}=\left(\psi_{l, q+1}, \psi_{l, q}, \ldots, \psi_{l, 1}\right)$ is an optimal SD-policy of the (MDP) for player $j$.

Proof of Claim 4. Let $\psi_{l, \leq q+1}: \Theta_{l, q+1}^{\prime} \rightarrow\{H, L\}$ such that

$$
\psi_{l, q+1}\left(\theta_{j}\right)= \begin{cases}H & \text { if } v_{j}^{*}\left(\theta_{j}\right)>U_{l}(L) \\ L & \text { if } v_{j}^{*}\left(\theta_{j}\right) \leq U_{l}(L) .\end{cases}
$$

Then $v\left(\cdot ; \psi_{l, \leq q+1}\right): \Theta_{l, \leq q+1}^{\prime} \rightarrow \mathbb{R} \cup\{-\infty\}$ solves the optimality equation of the (MDP) for player $j$. It follows from Theorem Puterman 2 that $\psi_{l, \leq q+1}$ is optimal.

By Claim (1), for any $\theta_{j}=(\mathbf{s}, q+1) \in \Theta_{l, q+1}^{\prime}$ with $s_{H h}<q+1$,

$$
v_{j}^{*}\left(\theta_{j}\right) \leq \max \left\{U_{l}(L), U_{l}(H)-\frac{\left(\bar{k}^{\text {fifo }}+1\right) c}{p}\right\}=U_{l}(L),
$$

so $\psi_{l, q+1}\left(\theta_{j}\right)=L$. This concludes the proof of Claim 4 .

To conclude the proof of Lemma A3, let us turn to Assumption A1. Thus far, we have constructed $\psi_{l}$, which ascribes a best-response for an $l$-round for any initial augmented state, given $\psi_{H}, \psi_{h}$, and Assumption Al applied to strategies of others. To conclude the proof, we need to guarantee that the $\psi_{l}$ we constructed satisfies Assumption Al. Take any stationary* strategy-profile $\Psi=\left(\psi_{H}, \psi_{L}, \psi_{h}, \psi_{l}\right)$ such that $\psi_{H}$ and $\psi_{h}$ satisfy (8), and $\psi_{l}$ and $\psi_{L}$ are constructed as described above. Suppose that both $L$-squares and $l$-rounds exist in the market in a period $t$ after a new pair arrives. We consider the case of $s_{H h}^{t} \geq 0$ (and omit an analogous proof for the case of $s_{H h}^{t}<0$ ). For any $l$-round, say player $i$, with rank $q_{i}>s_{H h}^{t}$, Claims 2 and 4 imply that player $i$ would demand $L$. The counterpart of Claims 2 and 4 for $L$-squares implies that every $L$-square, say player $j$, demands an $l$-round as $q_{j} \geq 1=1+s_{H h}^{-}$. Therefore, Assumption Al describes bestresponse behavior. This concludes the proof of Lemma A3.

Proof of Lemma 1. The proof follows directly from Lemmas A2 and A3 above.

Proof of Lemma 2. We show that the part (b) of Lemma A3 guarantees Lemma 2. Take a stationary* equilibrium $\Psi^{*}$ satisfying conditions (a) and (b) in Lemma A3. Initially, 
there is no agent waiting in the market. Suppose that both an $L$-square and an $l$-round are present in some period $t$, for the first time ever. Given (8) (and a similar condition for $\psi_{h}^{*}$ ), it must be that either (i) $s_{H}^{t} \geq 0, s_{h}^{t}=0, s_{L}^{t}=1$, and $s_{l}^{t}=s_{H}^{t}+s_{L}^{t}$, or (ii) $s_{H}^{t}=0$, $s_{h}^{t} \geq 0, s_{L}^{t}=s_{h}^{t}+s_{l}^{t}$, and $s_{l}^{t}=1$. In both instances, there exists an $L$-square who finds no available $h$-round and demands an $l$-round, and an $l$-round who finds no available $H$-square and demands an $L$-square. As such, one $(L, l)$ pair will be matched, and only incongruent pairs of agents (i.e., either $H$-squares and $l$-rounds, or $L$-squares and $h$ rounds) wait until period $t+1$. A similar argument shows that in any period in which an $L$-square and an $l$-round coexist, for the second time, third time, etc., one $(L, l)$ pair will be formed.

Proof of Proposition 3. First, the (signed) length of the $H$ - $h$ queue, denoted by $k_{H h}$, constitutes an ergodic Markov chain. Following arguments in the body of the paper, the unique steady-state distribution of $k_{H h}$ is the uniform distribution over $\left\{-\bar{k}^{\mathrm{dec}},-\bar{k}^{\mathrm{dec}}+\right.$ $\left.1, \ldots, \bar{k}^{\mathrm{dec}}\right\}$. At any time $t$, suppose that $k_{H h}^{t}>0$. Clearly, the queue has no $h$-rounds. As equal numbers of squares and rounds enter and exit the market, it must be that $k_{H h}+$ $k_{L}=k_{l}$. Lemma 2 guarantees that $k_{L}=0$, therefore, $k_{l}=k_{H h}$. An analogous argument follows for $k_{H h} \leq 0$.

Proof of Corollary 3. Whenever $c>\frac{p(1-p) U}{2}$, the optimal mechanism matches arriving agents immediately, $\bar{k}^{\mathrm{opt}}=0$, and $\bar{k}^{\mathrm{opt}} \leq \bar{k}^{\mathrm{dec}}$. Suppose then that $c<\frac{p(1-p) U}{2}$. We then have

$$
\sqrt{\frac{p(1-p) U}{2 c}}<\frac{p(1-p) U}{2 c} \leq \frac{p U}{2 c} \leq \frac{p\left(U_{H}(h)-U_{H}(l)\right)}{c} .
$$

and the result follows from the definitions of $\bar{k}^{\text {opt }}$ and $\bar{k}^{\mathrm{dec}}$.

\section{A.3 Proof regarding welfare comparisons}

Proof of Proposition 4. Part 1: As in the proof of Corollary $1, W^{\text {opt }}(c)-W^{\text {fifo }}(c)$ is differentiable at any $c<\frac{p(1-p) U}{2}$ such that $\bar{k}^{\text {opt }}, \bar{k}^{\text {fifo }} \notin \mathbb{Z}_{+}$. In a small neighborhood around any such $c$, the thresholds corresponding to both the optimal and discretionary thresholds are constant in $c$. Therefore,

$$
\frac{d\left(W^{\mathrm{opt}}(c)-W^{\mathrm{fifo}}(c)\right)}{d c}=-\frac{2 \bar{k}^{\mathrm{opt}}\left(\bar{k}^{\mathrm{opt}}+1\right)}{2 \bar{k}^{\mathrm{opt}}+1}+\frac{2 \bar{k}^{\mathrm{fifo}}\left(\bar{k}^{\mathrm{fifo}}+1\right)}{2 \bar{k}^{\text {fifo }}+1} \geq 0,
$$

where the inequality follows from $\bar{k}^{\text {fifo }} \geq \bar{k}^{\text {opt }}$ (Corollary 3 ). Furthermore, the proof of Corollary 1 implies that $W^{\text {opt }}(c)$ is continuous in $c$ and so $W^{\text {opt }}(c)-W^{\text {fifo }}(c)$ is increasing in any point $c$ for which $\bar{k}^{\text {fifo }} \notin \mathbb{Z}_{+}$. Let $\left\{d_{k}\right\}_{k=1}^{\infty}$ denote the decreasing sequence of costs such that $k=\frac{p\left(U_{H}(h)-U_{H}(l)\right)}{d_{k}}$. That is, cost $d_{k}$ corresponds to the maximal cost such that the equilibrium threshold is $k$ in the discretionary process under FIFO. For an arbitrary $k$, we will show that

$$
W^{\mathrm{opt}}\left(d_{k+1}\right)-W^{\mathrm{opt}}\left(d_{k}\right)>W^{\mathrm{fifo}}\left(d_{k+1}\right)-W^{\mathrm{fifo}}\left(d_{k}\right) .
$$


First, we focus on $W^{\mathrm{opt}}\left(d_{k+1}\right)-W^{\mathrm{opt}}\left(d_{k}\right)$. Note that $W^{\mathrm{opt}}(c)$ is piecewise linear and continuous in $c$. It follows that

$$
W^{\mathrm{opt}}\left(d_{k+1}\right)-W^{\mathrm{opt}}\left(d_{k}\right)=\int_{d_{k+1}}^{d_{k}} \frac{2 \bar{k}^{\mathrm{opt}}(c)\left(\bar{k}^{\mathrm{opt}}(c)+1\right)}{2 \bar{k}^{\mathrm{opt}}(c)+1} d c .
$$

Let $k^{0} \equiv\left\lfloor\sqrt{\frac{p(1-p) U}{2 d_{k}}}\right\rfloor$. For any $c \in\left[d_{k+1}, d_{k}\right], \bar{k}^{\mathrm{opt}}(c) \geq k^{0}$ and

$$
\frac{2 \bar{k}^{\mathrm{opt}}(c)\left(\bar{k}^{\mathrm{opt}}(c)+1\right)}{2 \bar{k}^{\mathrm{opt}}(c)+1}=\frac{1}{2}\left(\left(2 \bar{k}^{\mathrm{opt}}(c)+1\right)-\frac{1}{2 \bar{k}^{\mathrm{opt}}(c)+1}\right) \geq \frac{2 k^{0}\left(k^{0}+1\right)}{2 k^{0}+1} .
$$

Thus,

$$
\begin{aligned}
& W^{\mathrm{opt}}\left(d_{k+1}\right)-W^{\mathrm{opt}}\left(d_{k}\right) \\
& \quad \geq \int_{d_{k+1}}^{d_{k}} \frac{2 k^{0}\left(k^{0}+1\right)}{2 k^{0}+1} d c=\frac{2 k^{0}\left(k^{0}+1\right)}{2 k^{0}+1}\left(d_{k}-d_{k+1}\right) \\
& \quad=\frac{2 k^{0}\left(k^{0}+1\right)}{2 k^{0}+1} p\left(U_{H}(h)-U_{H}(l)\right)\left(\frac{1}{k}-\frac{1}{k+1}\right)=\frac{2 k^{0}\left(k^{0}+1\right)}{2 k^{0}+1} \frac{p\left(U_{H}(h)-U_{H}(l)\right)}{k(k+1)} .
\end{aligned}
$$

Next, we consider $W^{\text {fifo }}\left(d_{k+1}\right)-W^{\text {fifo }}\left(d_{k}\right)$. Denote by

$$
W(m, c) \equiv S_{\infty}-\frac{p(1-p) U}{2 m+1}-\frac{2 m(m+1) c}{2 m+1} .
$$

Note that

$$
\begin{aligned}
W^{\text {fifo }}\left(d_{k+1}\right)-W^{\text {fifo }}\left(d_{k}\right) & =W\left(k+1, d_{k+1}\right)-W\left(k, d_{k}\right) \\
& =W\left(k+1, d_{k+1}\right)-W\left(k+1, d_{k}\right)+W\left(k+1, d_{k}\right)-W\left(k, d_{k}\right) .
\end{aligned}
$$

We use the following two observations:

$$
W\left(k+1, d_{k+1}\right)-W\left(k+1, d_{k}\right)=\frac{2(k+2) p\left(U_{H}(h)-U_{H}(l)\right)}{k(2 k+3)},
$$

and

$$
\begin{aligned}
& W\left(k+1, d_{k}\right)-W\left(k, d_{k}\right) \\
& \quad=\frac{1}{(2 k+1)(2 k+3)}\left(2 p(1-p) U-\frac{4(k+1)^{2} p\left(U_{H}(h)-U_{H}(l)\right)}{k}\right) .
\end{aligned}
$$

Thus,

$$
\begin{aligned}
W^{\text {fifo }}\left(d_{k+1}\right)-W^{\text {fifo }}\left(d_{k}\right)= & \frac{2(k+2) p\left(U_{H}(h)-U_{H}(l)\right)}{k(2 k+3)}+\frac{2 p(1-p) U}{(2 k+1)(2 k+3)} \\
& -\frac{4(k+1)^{2} p\left(U_{H}(h)-U_{H}(l)\right)}{k(2 k+1)(2 k+3)} .
\end{aligned}
$$


To prove (10), it suffices to show that

$$
\frac{2 k^{0}\left(k^{0}+1\right)}{2 k^{0}+1} \frac{2 k+3}{k+1}>\frac{2 k}{2 k+1}+\frac{2 k}{2 k+1} \frac{(1-p) U}{U_{H}(h)-U_{H}(l)} .
$$

To prove the above inequality, we consider the following two cases:

1. $\left(k^{0} \geq 2\right)$ Note that

$$
\frac{U}{U_{H}(h)-U_{H}(l)}<\frac{\left(U_{H}(h)-U_{H}(l)\right)+\left(U_{h}(H)-U_{h}(L)\right)}{U_{H}(h)-U_{H}(l)}=2 .
$$

Since the left-hand side of (11) is increasing in $k^{0}$, for (11) to hold, it suffices that

$$
\frac{12}{5} \frac{2 k+3}{k+1}>\frac{2 k}{2 k+1}+\frac{4 k}{2 k+1}=\frac{6 k}{2 k+1},
$$

which holds for all $k$.

2. $\left(k^{0}=1\right)$ One sufficient condition for (11) using (12) is $\frac{4(2 k+3)}{3(k+1)}>\frac{6 k}{2 k+1}$, which holds for $k=1,2$, or 3 . Since $k^{0}=1$,

$$
\frac{p(1-p) U}{2 d_{k}}=\frac{(1-p) U k}{2\left(U_{H}(h)-U_{H}(l)\right)}<4 .
$$

Thus, another sufficient condition for (11) in this case is $\frac{4(2 k+3)}{3(k+1)}>\frac{2 k+16}{2 k+1}$, which holds for $k \geq 4$.

To construct the partition in the proposition, let $\bar{k}=\max \left\{k \mid d_{k} \geq \underline{c}\right\}$ and $\underline{k}=\min \{k \mid$ $d_{k}<\bar{c}$ \}. Now define $c_{1}=\underline{c}, c_{M}=\bar{c}$. If $\underline{k}=\bar{k}$, set $M=2$ and the partition has only one atom. Otherwise, if $\underline{k}<\bar{k}$, set $M=\bar{k}-\underline{k}+2$ and $c_{i}=d_{\bar{k}-i+1}$ for $i=2, \ldots, M-1$.

Part 2: Notice that

$$
\lim _{c \rightarrow 0}\left(W^{\text {opt }}(c)-W^{\text {fifo }}(c)\right)=\lim _{c \rightarrow 0} \Psi(c)-\lim _{c \rightarrow 0} \Theta(c)=p\left(U_{H}(h)-U_{H}(l)\right) .
$$

In particular, for sufficiently small $c, W^{\text {opt }}(c)-W^{\text {fifo }}(c)$ is increasing in both $p$ and $U_{H}(h)-U_{H}(l)$, as needed.

\section{A.4 Proofs regarding the LIFO protocol}

Proof of Lemma 3. For an $H$-square, say player $i$, let $\theta_{i}=\left(\mathbf{s}, q_{i}\right)$ denote her augmented state, where $q_{i}$ now denotes her rank under the LIFO protocol. A threshold strategy is a SD-strategy $\psi_{H}$ such that, with some $\bar{k}_{H} \in \mathbb{Z}_{+}$,

$$
\psi_{H}\left(\theta_{i}\right)= \begin{cases}h & \text { if } q_{i} \leq \bar{k}_{H} \\ l & \text { if } q_{i}>\bar{k}_{H}+1 .\end{cases}
$$

Similarly, we define a threshold strategy for $h$-rounds with the threshold denoted by $\bar{k}_{h}$. Suppose that all $H$-squares play a threshold strategy $\psi_{H}$ with threshold $\bar{k}_{H} \in \mathbb{Z}_{+}$. We 
use an absorbing Markov chain to compute the expected total payoff for an $H$-square, say player $i$, whose augmented state is $\theta_{i}^{t}=\left(\mathbf{s}^{t}, q_{i}^{t}\right)$ in some period $t$. The event time $\tau$ starts from 0 and increases for each arrival of an incongruent pair. ${ }^{45}$ In expectation, an increment of $\tau$ takes $\frac{1}{2 p(1-p)}$ periods. The state space of the absorbing Markov chain is $\left\{1,2,3, \ldots, \bar{k}_{H}, h, l\right\}$ where integer transient states denote player $i$ 's ranking $q_{i}^{\tau}$ ( 1 if there are no $H$-squares who arrived after $i$ that are waiting), and each of the two absorbing states $h$ and $l$ denote the type of player $i$ 's match partner. The matrix of transition probabilities $p_{i j}$ from state $i$ to $j$ is

$P=\left[\begin{array}{cc}Q & R \\ \mathbf{0} & I\end{array}\right], \quad$ where $Q=\left[\begin{array}{cccc}0 & 1 / 2 & \cdots & 0 \\ 1 / 2 & 0 & \cdots & 0 \\ \vdots & \vdots & \ddots & \vdots \\ 0 & \cdots & 0 & 1 / 2 \\ 0 & \cdots & 1 / 2 & 0\end{array}\right], R=\left[\begin{array}{cc}1 / 2 & 0 \\ 0 & 0 \\ \vdots & \vdots \\ 0 & 1 / 2,\end{array}\right]$, and $I=\left[\begin{array}{ll}1 & 0 \\ 0 & 1\end{array}\right]$.

$Q$ represents the transitions between transient states. For any $1<k<\bar{k}_{H}$, the state changes upon an arrival of either an $(H, l)$ or a $(L, h)$ pair, each of which occurs with conditional probability $1 / 2$. $R_{11}$ represents the probability of a transition from $q_{i}=1$ to an absorbing state $h$ caused by an arrival of a $(L, h)$ pair. $R_{\bar{k}_{H} 2}$ represents the transition from $q_{i}=\bar{k}_{H}$ to an absorbing state $l$ caused by an arrival of an $(H, l)$ pair. Let $N \equiv\left(I_{\bar{k}_{H}}-Q\right)^{-1}, T \equiv N \cdot \mathbf{1}$, and $L \equiv N R$. The absorbing Markov chain with initial state $k \in\left\{1,2, \ldots, \bar{k}_{H}\right\}$ is absorbed in $T_{k}$ expected number of steps. It is absorbed by state $h$ (or $l$ ) with probability $L_{k h}$ (or $L_{k l}$, resp.). It is easy to verify that $N$ is a symmetric matrix with $N_{i j}=\frac{2 j(k-i+1)}{k+1}$ for all $i \geq j, T_{k}=T_{\bar{k}_{H}+1-k}=\sum_{i=1}^{k}\left(\bar{k}_{H}-2(i-1)\right)$ for all $k \leq \bar{k}_{H} / 2$, and $L_{k 2}=1-L_{k 1}=k /\left(\bar{k}_{H}+1\right)$ for $k=1, \ldots, \bar{k}_{H}$. The expected total payoff for player $i$ in period $t$ with initial condition $q_{i}^{t}=k$ is

$$
L_{k 1} U_{H}(h)+L_{k 2} U_{H}(l)-T_{k} \frac{c}{2 p(1-p)} .
$$

This payoff is strictly decreasing in $k$, implying that an $l$-round with rank $\bar{k}_{H}$ has the highest incentive to deviate from $\psi_{H}$ by demanding $l$, among all $H$-squares who are supposed to demand $h$ according to $\psi_{H}$. The total expected payoff for player $i$ with $q_{i}^{t}=\bar{k}_{H}$ is

$$
\frac{1}{\bar{k}_{H}+1} U_{H}(h)+\frac{\bar{k}_{H}}{\bar{k}_{H}+1} U_{H}(l)-\frac{\bar{k}_{H} c}{2 p(1-p)} .
$$

This payoff is strictly decreasing in $\bar{k}_{H}$. Thus, there exists a maximum threshold $\bar{k}^{\text {lifo }}$ such that player $i$ 's payoff exceeds $U_{H}(l) .{ }^{46}$ After some algebraic steps, one can verify

\footnotetext{
${ }^{45}$ An arrival of $(H, h)$ or $(L, l)$ does not change player $i$ 's position in line. In particular, if an $(H, h)$ pair arrives, the new players match with each other immediately under LIFO.

${ }^{46}$ The last payoff is never equal to $U_{H}(l)$ because of the regularity assumption.
} 
that

$$
\bar{k}^{\text {lifo }} \equiv\left\lfloor\sqrt{\frac{2 p(1-p)\left(U_{H}(h)-U_{H}(l)\right)}{c}+\frac{1}{4}}-\frac{1}{2}\right\rfloor .
$$

Next, we show that if $\Psi=\left(\psi_{H}, \psi_{L}, \psi_{h}, \psi_{l}\right)$ is a stationary* equilibrium in which $\psi_{H}$ (and $\psi_{h}$ ) is a threshold strategy with a threshold $\bar{k}_{H}$ (resp., $\bar{k}_{h}$ ), then $\bar{k}_{H}=\bar{k}_{h}=\bar{k}^{\text {lifo }}$.

(i) Suppose, toward a contradiction, that $\bar{k}_{H}>\bar{k}^{\text {lifo }}$. Take any $H$-square $i$ whose augmented state in some period $t$ satisfies $q_{i}^{t}=\bar{k}_{H}$. Her expected total payoff from period $t$ by playing $\psi_{H}$ is strictly lower than $U_{H}(l)$. Therefore, player $i$ has an incentive to deviate and demand an $l$-round.

(ii) Suppose, toward a contradiction, that $\bar{k}_{H}<\bar{k}^{\text {lifo }}$. Take any $H$-square $i$ whose augmented state in some period $t$ satisfies $q_{i}^{t}=\bar{k}$. We will show that player $i$ has an incentive to deviate and use the threshold $\bar{k}_{H}+1$ instead of $\bar{k}_{H}$ perpetually until matching. Consider an absorbing Markov chain with the state space $\left\{1,2, \ldots, \bar{k}_{H}, \bar{k}_{H}+1, h\right\}$ such that each integer transient state denotes player $i$ 's ranking. The absorbing state $h$ represents the only possible match partner for $i$, a match with an $h$-round. The queue for $H$-squares never exceeds the threshold $\bar{k}_{H}+1$, because all other $H$-squares use the threshold $\bar{k}_{H}$. Therefore, player $i$ will never match with an $l$-round. The transition probability matrix is

$$
P=\left[\begin{array}{cc}
Q & R \\
\mathbf{0} & 1
\end{array}\right], \quad \text { where } Q=\left[\begin{array}{cccccc}
0 & 1 / 2 & 0 & \cdots & 0 & 0 \\
1 / 2 & 0 & 1 / 2 & \cdots & 0 & 0 \\
\vdots & \vdots & \vdots & \ddots & \vdots & \vdots \\
0 & 0 & 0 & \cdots & 1 / 2 & 1 / 2
\end{array}\right] \text { and } R=\left[\begin{array}{c}
1 / 2 \\
0 \\
\vdots \\
0
\end{array}\right]
$$

To understand $Q_{\left(\bar{k}_{H}+1\right)\left(\bar{k}_{H}+1\right)}=1 / 2$, suppose that player $i$ 's augmented state in some period $\tau$ satisfies $q_{i}^{\tau}=\bar{k}_{H}+1$. If an $(H, l)$ pair arrives in the following period, one of other $H$-squares who play $\psi_{H}$ with threshold $\bar{k}_{H}$ demands an $l$-round and leaves the market. Player $i$ 's rank (i.e., the state in the absorbing Markov chain) will remain at $\bar{k}_{H}+1$. Let $N \equiv\left(I_{\bar{k}_{H}+1}-Q\right)^{-1}$ and $T \equiv N \cdot 1$. The absorbing Markov chain with initial state $k \in$ $\left\{1,2, \ldots, \bar{k}_{H}+1\right\}$ is absorbed by state $h$ in $T_{k}$ expected number of steps. It is easy to verify that $N$ is a symmetric matrix with $N_{i j}=2 j$ for all $i \geq j$, and $T_{k}=2 \sum_{i=1}^{k}\left(\bar{k}_{H}+2-i\right)$. Therefore, when player $i$ 's augmented state in period $\tau$ is $q_{i}^{\tau}=\bar{k}_{H}+1$, she would deviate from $\psi_{H}$ by increasing the threshold to $\bar{k}_{H}+1$ permanently because

$$
U_{H}(h)-\frac{\left(\bar{k}_{H}+1\right)\left(\bar{k}_{H}+2\right) c}{2 p(1-p)} \geq U_{H}(h)-\frac{\left(\bar{k}^{\text {lifo }}\right)\left(\bar{k}^{\text {lifo }}+1\right) c}{2 p(1-p)}>U_{H}(l) .
$$

Thus, a stationary* strategy $\psi_{H}$ with threshold $\bar{k}_{H}<\bar{k}^{\text {lifo }}$ cannot be a stationary* equilibrium strategy.

Proof of Lemma 4. First, consider the decisions of $H$-squares. Suppose that $H$ squares play a stationary* strategy $\psi_{H}$ with threshold $\bar{k}^{\text {lifo }}$. We prove that, for each $H$-square, say player $i, \psi_{i}=\psi_{H}$ is an optimal policy of the (MDP) (without restrictions 
on her initial state), defined by other $H$-squares' strategy $\psi_{H}$. It follows that $\psi_{H}$ is each $H$-square's best-response. Given any initial augmented state $\theta_{i}=\left(\mathbf{s}, q_{i}\right)$ and other $H$ squares' strategy $\psi_{H}$, define the value of policy $\psi_{i}\left(=\psi_{H}\right)$ as

$$
v_{i}\left(\theta_{i} ; \psi_{i}, \psi_{H}\right) \equiv E_{\psi_{i}}\left[\sum_{t=0}^{\infty} u_{i}\left(\psi_{i}\left(\theta_{i}^{t}\right), \theta_{i}^{t}\right): \theta_{i}^{0}=\theta_{i}\right] .
$$

From equation (13), we obtain that

$$
v_{i}\left(\theta_{i} ; \psi_{i}, \psi_{H}\right)=\left\{\begin{array}{l}
U_{H}(h) \quad \text { if } q_{i} \leq s_{h} \\
\left(1-\frac{k}{\bar{k}^{\text {lifo }}+1}\right) U_{H}(h)+\frac{k}{\bar{k}^{\text {lifo }}+1} U_{H}(l)-T_{k} \frac{c}{2 p(1-p)} \\
\quad \text { if } k \equiv q_{i}-s_{h} \in\left\{1, \ldots, \bar{k}^{\text {lifo }}\right\} \\
U_{H}(l) \quad \text { if } q_{i}-s_{h}>\bar{k}^{\text {lifo }} .
\end{array}\right.
$$

It is easy to verify that $v_{i}\left(\theta_{i} ; \psi_{i}, \psi_{H}\right)$ solves the optimality equation

$$
v\left(\theta_{i}\right)=\max _{d_{i} \in\left\{h, l_{\}}\right.}\left[u_{i}\left(d_{i}, \theta_{i}\right)+\sum_{\theta_{i}^{\prime} \in \Theta_{i}} p\left(\theta_{i}^{\prime}: \theta_{i}, d_{i}\right) V\left(\theta_{i}^{\prime}\right)\right] \text { for all } \theta_{i} \in \Theta_{i}
$$

Then, by Theorem Puterman 2 appearing in the Appendix, $\psi_{i}$ is an optimal SD-policy of the Markov decision problem, defined by other $H$-squares' stationary* strategy $\psi_{H}$. Let us now turn to the $l$-rounds' decisions. Suppose that $H$-squares (and $h$-rounds) play the stationary* strategy with threshold $\bar{k}^{\text {lifo }}$. Then, only if an $(H, l)$ pair arrives, there may exist an $H$-square (in fact, exactly one $H$-square) who may demand an $l$-round, and she matches with the last arriving $l$-round. Thus, if an $l$-round remains unmatched after the first period at the market, he will not match with an $H$-square ever again. Thus, every $l$-round has an incentive to leave immediately. Therefore, we have that

$$
\psi_{l}\left(\mathbf{s}, q_{i}\right)= \begin{cases}h & \text { if } s_{H h} \geq \bar{k}^{\text {lifo }}+1 \text { and } q_{i}=1 \\ l & \text { otherwise }\end{cases}
$$

is a best-response for all $l$-rounds with any initial augmented state.

Proof of Corollary 5. 1. Ignoring integer constraints, we have

$$
\begin{aligned}
\lim _{c \rightarrow 0} \frac{\bar{k}^{\text {opt }}}{\bar{k}^{\text {lifo }}} & =\lim _{c \rightarrow 0} \frac{\sqrt{\frac{p(1-p) U}{2 c}}}{\sqrt{\frac{2 p(1-p)\left(U_{H}(h)-U_{H}(l)\right)}{c}+\frac{1}{4}}-\frac{1}{2}}=\frac{\sqrt{p(1-p) U}}{\sqrt{4 p(1-p)\left(U_{H}(h)-U_{H}(l)\right)}} \\
& =\frac{1}{2} \sqrt{\frac{U}{U_{H}(h)-U_{H}(l)}}<1,
\end{aligned}
$$


where the last inequality is due to $U<4\left(U_{H}(h)-U_{H}(l)\right)$ under our symmetry assumption. Furthermore, we have $\bar{k}^{\text {lifo }}<\bar{k}^{\text {fifo }}$ if and only if

$$
\sqrt{\frac{2 p(1-p)\left(U_{H}(h)-U_{H}(l)\right)}{c}+\frac{1}{4}}-\frac{1}{2}<\frac{p\left(U_{H}(h)-U_{H}(l)\right)}{c} .
$$

Let $x \equiv \frac{2 p(1-p)\left(U_{H}(h)-U_{H}(l)\right)}{c}$ and $z \equiv \frac{p\left(U_{H}(h)-U_{H}(l)\right)}{c}$, so that inequality (14) is equivalent to $\sqrt{x+\frac{1}{4}}<z+\frac{1}{2}$, or $x<z^{2}+z$. Thus, (14) is satisfied if and only if

$$
\frac{2 p(1-p)\left(U_{H}(h)-U_{H}(l)\right)}{c}<\frac{p^{2}\left(U_{H}(h)-U_{H}(l)\right)^{2}}{c^{2}}+\frac{p\left(U_{H}(h)-U_{H}(l)\right)}{c},
$$

or equivalently

$$
1-2 p<\frac{p\left(U_{H}(h)-U_{H}(l)\right)}{c} .
$$

Therefore, if $p \geq \frac{1}{2}$, then $1-2 p \leq 0$, and $\bar{k}^{\text {lifo }}<\bar{k}^{\text {fifo }}$ for any $c>0$. If $p<\frac{1}{2}$, then $1-2 p>0$, and $\bar{k}^{\text {lifo }}<\bar{k}^{\text {fifo }}$ for any $c<\frac{p\left(U_{H}(h)-U_{H}(l)\right)}{1-2 p}$.

2. As $\bar{k}^{\text {lifo }}=\sqrt{\frac{2 p(1-p)\left(U_{H}(h)-U_{H}(l)\right)}{c}+\frac{1}{4}}-\frac{1}{2}$ and

$$
W^{\text {lifo }}(c)=S_{\infty}-\frac{p(1-p) U}{2 \bar{k}^{\text {lifo }}+1}-\frac{2 \bar{k}^{\text {lifo }}\left(\bar{k}^{\text {lifo }}+1\right)}{2 \bar{k}^{\text {lifo }}+1} c,
$$

it is easy to verify that $\lim _{c \rightarrow 0} W^{\text {lifo }}(c)=S_{\infty}$.

\section{REFERENCES}

Akbarpour, Mohammad, Shengwu Li, and Shayan Oveis Gharan (2020), “Thickness and information in dynamic matching markets.” Journal of Political Economy 128, 783-815. [1224, 1233]

Anderson, Ross, Itai Ashlagi, David Gamarnik, and Yash Kanoria (2017), "Efficient dynamic barter exchange." Operations Research, 65, 1446-1459. [1225]

Ashlagi, Itai, Maximilien Burq, Patrick Jaillet, and Vahideh H. Manshadi (2019), "On matching and thickness in heterogeneous dynamic markets." Operations Research, 67, 927-949. [1225]

Ashlagi, Itai, Patrick Jaillet, Vahideh H. Manshadi, and Michael A. Rees (2014), "Kidney exchange in dynamic sparse heterogenous pools." Unpublished paper, Department of Economics, MIT. [1225]

Baccara, Mariagiovanna, Allan Collard-Wexler, Leonardo Felli, and Leeat Yariv (2014), "Child-adoption matching: Preferences for gender and race." American Economic Journal: Applied Economics, 6, 133-158. [1221, 1222, 1227] 
Baccara, Mariagiovanna, SangMok Lee, and Leeat Yariv (2018). "Optimal dynamic matching.” Working paper, CEPR Discussion Paper No. DP12986. [1226, 1228, 1242]

Becker, Gary S. (1974), "A theory of marriage: Part II." Journal of Political Economy, 82, S11-S26. [1226]

Bloch, Francis and David Cantala (2017), "Dynamic assignment of objects to queuing agents.” American Economic Journal: Microeconomics, 9, 88-122. [1225]

Burdett, Ken and Melvyn G. Coles (1997), “Marriage and class.” Quarterly Journal of Economics, 112, 141-168. [1225]

Doval, Laura (2020), "Dynamically stable matching." Unpublished paper, Division of Humanities and Social Science, California Institute of Technology. [1225]

Doval, Laura and Balász Szentes (2019), "On the efficiency of queueing in dynamic matching markets.” Working paper, Division of the Humanities and Social Sciences, California Institute of Technology. [1224]

Eeckhout, Jan (1999), "Bilateral search and vertical heterogeneity." International Economic Review, 40, 869-887. [1225]

Ferdowsian, Andrew, Muriel Niederle, and Leeat Yariv (2020), "Decentralized matching with aligned preferences.” Working paper, Department of Economics, Princeton University. [1225]

Gjertson, David W. (2004), "Explainable variation in renal transplant outcomes: A comparison of standard and expanded criteria donors." Clinical Transplants, 303-314. [1227]

Gurvich, Itai and Amy Ward (2014), "On the dynamic control of matching queues." Stochastic Systems, 4, 479-523. [1224]

Haeringer, Guillaume and Myrna Wooders (2011), “Decentralized job matching." International Journal of Game Theory, 40, 1-28. [1225]

Hall, Robert E. and Alan B. Krueger (2012), "Evidence on the incidence of wage posting, wage bargaining, and on-the-job search." American Economic Journal: Macroeconomics, 4, 56-67. [1226]

Hassin, Refael (1985), "On the optimality of first come last served queues." Econometrica, 53, 201-202. [1225, 1242]

Hitch, Gunter J., Ali Hortacsu, and Dan Ariely (2010), "Matching and sorting in online dating." American Economic Review, 100, 130-163. [1227]

$\mathrm{Hu}$, Ming and Yun Zhou (2018), "Dynamic type matching." Working paper, Rotman School of Management Working Paper No. 2592622. [1224]

Kemeny, John G. and J. Laurie Snell (1960), Finite Markov Chains. Van Nostrand Publishing Company, New York City. [1244]

Leshno, Jacob (2019), "Dynamic matching in overloaded waiting lists.” Unpublished paper, SSRN 2967011. [1225] 
Loertscher, Simon, Ellen V. Muir, and Peter G. Taylor (2018), “Optimal market thickness and clearing.” Unpublished paper, Department of Economics, University of Melbourne. [1224]

Margaria, Chiara (2019), “Queueing to learn.” Unpublished paper, Department of Economics, Boston University. [1242]

Naor, Pinhas (1969), “The regulation of queue size by levying tolls.” Econometrica, 37, 15-24. [1225]

Øien, Cecilia M., Anna V. Reisaeter, Torbjørn Leivestad, Friedo W. Dekker, and Pål-Dag Line (2007), "Living donor kidney transplantation: The effects of donor age and gender on short- and long-term outcomes." Transplantation, 83, 600-606. [1227]

Ortoleva, Pietro, Evgenii Safonov, and Leeat Yariv (2020), "Who cares more? Allocation with diverse preference intensities." Unpublished paper, Department of Economics and Woodrow Wilson School, Princeton University. [1225]

Pais, Joana V. (2008), "Incentives in decentralized random matching markets." Games and Economic Behavior, 64, 632-649. [1225]

Platz, Trine Tornøe and Lars Peter Østerdal (2017), “The curse of the first-in-first-out queue discipline." Games and Economic Behavior, 104, 165-176. [1242]

Puterman, Martin L. (2005), Markov Decision Processes: Discrete Stochastic Dynamic Programming. Wiley Series in Probability and Statistics. Wiley, Hoboken, New Jersey. [1229, 1252, 1259, 1260, 1264]

Rogerson, Richard, Robert Shimer, and Randall Wright (2005), "Search-theoretic models of the labor market: A survey.” Journal of Economic Literature, 43, 959-988. [1225]

Ross, Sheldon M. (2014), Introduction to Stochastic Dynamic Programming (Z. W. Birnbaum and E. Lukacs, eds.). Academic Press, New York, New York. [1229]

Satterthwaite, Mark and Artyom Shneyerov (2007), "Dynamic matching, two-sided incomplete information, and participation costs: Existence and convergence to perfect competition." Econometrica, 75, 155-200. [1225]

Schummer, James (2019), “Influencing waiting lists.” Unpublished paper, MEDS, Kellogg School of Management, Northwestern University. [1225]

Stein, Rob (2011), "Under kidney transplant proposal, younger patients would get the best organs.” The Washington Post, 24. [1227]

Taylor, Curtis (1995), "The long side of the market and the short end of the stick: Bargaining power and price formation in buyers', sellers', and balanced markets.” Quarterly Journal of Economics, 110, 837-855. [1225]

Ünver, Utku (2010), “Dynamic kidney exchange.” Review of Economic Studies, 77, 372414. [1224] 
Zenios, Stefanos A. (1999), "Modeling the transplant waiting list: A queueing model with reneging.” Queueing Systems, 31, 239-251. [1224]

Co-editor Thomas Mariotti handled this manuscript.

Manuscript received 30 April, 2019; final version accepted 25 October, 2019; available online 1 November, 2019. 